\title{
Electron irradiation induced aging effects on radiative recombination properties of quadruple cation organic-inorganic perovskite layers
}

\author{
Pierfrancesco Aversa ${ }^{1} \cdot$ Senol Öz ${ }^{2,3} \cdot$ Eunhwan Jung ${ }^{2}$ - Olivier Plantevin ${ }^{4} \cdot$ Olivier Cavani $^{1} \cdot$ Nadège Ollier $^{1}$. \\ Jean-Eric Bourée ${ }^{5} \cdot$ Bernard Geffroy $^{5,6} \cdot$ Tsutomu Miyasaka $^{3} \cdot$ Sanjay Mathur ${ }^{2} \cdot$ Catherine Corbel $^{1}$
}

Received: 9 December 2019 / Accepted: 30 March 2020 / Published online: 29 April 2020

(C) The Author(s) 2020

\begin{abstract}
Understanding the role of defects in hybrid organic inorganic perovskites (HOIPs) is critically important to engineer the stability and performance of photovoltaic devices based on HOIPs. Recent reports on multi-cation compositions of general formula $\left(\mathrm{A}^{1}, \mathrm{~A}^{\mathbf{2}}, \mathrm{A}^{3}, \mathrm{~A}^{4}\right) \mathrm{Pb}\left(\mathrm{X}^{\mathbf{1}}, \mathrm{X}^{\mathbf{2}}, \mathrm{X}^{3}\right)_{3}$, where the $\mathrm{A}$ sites can be occupied by a distribution of 2-4 metallic/ organic cations and $\mathrm{X}$ sites with halide anions have shown stabilization effects against the well-known methyl ammonium lead triiodide $\left(\mathrm{CH}_{3} \mathrm{NH}_{3} \mathrm{PbI}_{3}\right)$, although the underlying mechanism is not fully elucidated. Herein, polycrystalline layers of $4 \mathrm{APb}(\mathrm{IBr})_{3}$ perovskite, where $\mathrm{A}$ is occupied by a combination of $\mathrm{Cs}^{+}$(cesium ion), $\mathrm{GA}^{+}$(guanidinium), $\mathrm{MA}^{+}$ (methylammonium), and $\mathrm{FA}^{+}$(formamidinium) ions were synthesized. To gain insight on the role of intrinsic defects, electron irradiation was used for introducing point defects in a controlled way in the quadruple-cation HOIPs. Our results show that the engineered defects in perovskites strongly influenced the absorption, photoluminescence, and time-resolved photoluminescence of these materials, probably due to introduction of additional energy levels that modify electronic and light emitting properties of the material. Furthermore, the irradiation-induced defects were found to strongly affect the aging behavior of HOIPs and modify their radiative recombination properties.
\end{abstract}

Keywords Quadruple-cation hybrid perovskites $\cdot$ Defects $\cdot$ Electron irradiation $\cdot$ Photoluminescence $\cdot$ Aging effects

Sanjay Mathur

sanjay.mathur@uni-koeln.de

Catherine Corbel

catherine.corbel@polytechnique.edu

1 LSI, CEA/DRF/IRAMIS, Ecole Polytechnique, CNRS, lnstitut Polytechnique de Paris, 91128 Palaiseau, France

2 Institute of Inorganic Chemistry, University of Cologne, Greinstrasse 6, 50939 Cologne, Germany

3 Graduate School of Engineering, Toin University of Yokohama, 1614 Kuroganecho, Aoba, Yokohama, Kanagawa 225-8503, Japan

4 Laboratoire de Physique des Solides, CNRS, Université Paris-Saclay, 91405 Orsay, France

5 LPICM, CNRS, Ecole Polytechnique, Institut Polytechnique de Paris, Route de Saclay, 91128 Palaiseau, France

6 CEA, CNRS, NIMBE, LICSEN, Université Paris-Saclay, 91191 Gif-sur-Yvette, France

\section{Introduction}

Due to their unique optoelectronic properties (high absorption coefficient in the visible range, long charge carrier lifetime, high carrier mobility) and their simple processability through both solution and gas phase techniques, the hybrid organic inorganic halide perovskites (HOIPs) have witnessed a seminal growth in their photovoltaic efficiencies reaching the performance of standard semiconductors like silicon [1]. Although a large body of data has become available on perovskite-based photovoltaic devices mostly based on compositional variations (isoelectronic substitutions), the perovskite technology suffers from a number of stress factors, such as instability of HOIPs under illumination (both solar and UV), atmospheric conditions (moisture and oxygen), and interfacial reactions (ion migration), which deteriorate their device performance and efficiency. In this context, the role of native vacancy defects has been discussed as a possible cause for solar cell degradation, carrier lifetime reduction as well as for the hysteresis in the current-voltage curve under illumination. Defect-related trap states can act as nonradiative 
recombination centers, limiting photovoltaic devices performances. A full understanding of the nature of the defectinduced electronic states, as well as of their role on device performance and degradation, is still under debate [2].

The multi-cation HOIPs with the general formula $\left(\mathrm{A}^{1}, \mathrm{~A}^{2}, \mathrm{~A}^{3}, \mathrm{~A}^{4}\right) \mathrm{Pb}\left(\mathrm{X}^{1}, \mathrm{X}^{2}, \mathrm{X}^{3}\right)_{3}$, where the $\mathrm{A}$ sites can be occupied by a distribution of 2 to 4 metallic/organic cations and $X$ sites with halide anions, have recently demonstrated higher structural and environmental stability than the parent compound methylammonium lead triiodide $\left(\mathrm{CH}_{3} \mathrm{NH}_{3} \mathrm{PbI}_{3}\right.$; $\mathrm{MAPbI}_{3}$ ). The substitutional mixing on cation and anion sites allows to find the optimal stoichiometry enabling structural stability of the perovskite materials. This can improve perovskite stability at high temperatures under photovoltaic operational conditions and its sensitivity to moisture and humidity [3].

In this work, polycrystalline films of a quadruple cation perovskite, (GACsMAFA) $\mathrm{Pb}(\mathrm{IBr})_{3}$, were synthesized. It was shown in recent studies $[4,5]$ that this quadruple cation-based halide perovskite composition is successfully integrated in solar cell technologies yielding stable efficiencies (20\%) under operational conditions. In order to understand the perovskite defect properties, and to verify the concept of defect tolerance and self-healing associated to HOIPs [2], electron irradiation was used to introduce defects in a controlled manner in the quadruple-cation HOIPs films. Onemegaelectronvolt electron irradiation resistance $[6,7]$ has been reported for $\mathrm{MAPbI}_{3}$-based solar cells. Moreover, implantation and irradiation resistance to protons with energies ranging between $50 \mathrm{keV}$ and $68 \mathrm{MeV}$ in $\mathrm{MAPbI}_{3}$ [6-8] and (FAMA) $\mathrm{Pb}(\mathrm{IBr})_{3}[6]$ perovskite-based solar cells, respectively, has also been demonstrated. Recently, the effect of photoluminescence properties of $30 \mathrm{keV} \mathrm{He}$ ion irradiated $\mathrm{MAPbI}_{3}$ perovskite layers [9] has been reported. Particle irradiation can open new roads for defect engineering in HOIPs as well as it can help to understand in a more complete way perovskite fundamental properties.

To address the question whether, depending on its nature and concentration, the defect population in (GACsMAFA) $\mathrm{Pb}(\mathrm{IBr})_{3}$ layers plays any role in their stability and carrier recombination, this work for the first time uses electron irradiation to introduce intrinsic involving vacancies and interstitials defects. For this purpose, a set of quadruple layers of the chemical composition (GACsMAFA) $\mathrm{Pb}(\mathrm{IBr})_{3}$ were deposited on glass substrates and irradiated with $1 \mathrm{MeV}$ electrons introduced from the glass side and with an increasing fluence. Electrons were delivered by a Pelletron accelerator from National Electrostatic Corporation (NEC) in the SIRIUS facility located in Laboratoire des Solides Irradiés (LSI), Ecole Polytechnique - Palaiseau. The choice of performing irradiation from the glass side was to simulate the same geometry as applied for the sunlight illumination of solar cells.
To examine whether electron irradiation results in any change of the bandgap and of conduction/valence band edges, absorption measurements were performed on the complete set of both reference and irradiated HOIP films. The carrier radiative recombination and excited carrier lifetimes upon irradiation were monitored using photoluminescence (PL) and time-resolved photoluminescence (TRPL) studies. With PL, the response of the (GACsMAFA) $\mathrm{Pb}(\mathrm{IBr})_{3}$ perovskite film under continuous laser illumination was also investigated for comparison with the so called light soaking effect already observed in $\mathrm{MAPbI}_{3}[10,11]$ and with reference to the photoresponse of other mixed halide perovskites [12].

Our experimental findings evidently show that intrinsic defects affect the radiative recombination properties in (GACsMAFA) $\mathrm{Pb}(\mathrm{IBr})_{3}$ perovskite films. This effect was systematically monitored at increasing aging time after irradiation. The correlation between the absorption and PL emission spectra after post-irradiation aging indicated that the radiative recombination involves electronic states located near the band edges rather than deep levels inside the bandgap.

\section{Experimental methods}

F a b r ic at i o n proced ure F i v e $\mathrm{GA}_{0.015} \mathrm{Cs}_{0.046} \mathrm{MA}_{0.152} \mathrm{FA}_{0.787} \mathrm{~Pb}\left(\mathrm{I}_{0.815} \mathrm{Br}_{0.185}\right)_{3}$ ((GACsMAFA) $\mathrm{Pb}(\mathrm{IBr})_{3}$ ) perovskite films (set-18) were fabricated in a same preparation run on $12 \times 12 \times 1 \mathrm{~mm}^{3}$ glass substrates in a nitrogen filled glovebox. In addition, one reference film (rf17) was fabricated in another preparation run under the same conditions as the set-18. The substrates were treated with UV ozone for $15 \mathrm{~min}$. The precursor solution was prepared by dissolving GAI (0.02 M), CsI (0.09 M), MABr (0.2 M), FAI (1.1 M), $\mathrm{PbI}_{2}(1.2 \mathrm{M})$, and $\mathrm{PbBr}_{2}(0.3 \mathrm{M})$ in anhydrous dimethylformamide (DMF): dimethyl-sulfoxide (DMSO) (4:1, $\mathrm{v} / \mathrm{v}$ ) mixed solvent. The stoichiometry for the solid composition (see above) is derived from the solution composition. The perovskite precursor solution was spin coated onto glass substrates via three step spin coating at $2000 \mathrm{rpm}, 5000 \mathrm{rpm}$, and $6000 \mathrm{rpm}$ for $10 \mathrm{~s}, 25 \mathrm{~s}$, and $25 \mathrm{~s}$, respectively, with toluene anti-solvent dripped $45 \mathrm{~s}$ after the start of the spin coating process. The perovskite layer was annealed at $100{ }^{\circ} \mathrm{C}$ for $1 \mathrm{~h}$ in a nitrogen filled glovebox. Before any treatment, the set-18 layers were stored in dark and under dry $\mathrm{N}_{2}$ atmosphere for 1 week. The rf-17 layer was characterized in its pristine state immediately after preparation.

Controlled defect introduction via electron irradiation Four layers of the set-18 were individually irradiated from the glass substrate side with the $1-\mathrm{MeV}$ electron beam delivered by the SIRIUS Pelletron accelerator (LSI-Polytechnique). Each layer was successively mounted on a $\mathrm{Cu}$ sample holder. Then, the sample holder was fixed in a specifically designed irradiation 
chamber kept at room temperature $(300 \mathrm{~K})$ and filled with $\mathrm{N}_{2}$ atmosphere. A thermocouple put on the sample holder was used to control that no heating was produced during each layer irradiation.

The electron beam size diameter on the holder is about $17 \mathrm{~mm}$ with an intensity that is rather homogeneous where the $12 \times 12 \mathrm{~mm}^{2}$ layer is mounted. The glass thickness being the same for each layer, this ensures that the dose deposition is rather homogeneous in the layers. Each irradiation is performed at the same beam intensity. During each irradiation, the beam intensity is continuously monitored and kept at this same constant value. Consequently, each layer is irradiated with the same electron flux. Before the irradiations, a calibration procedure is performed to relate the electron beam intensity monitored during irradiation to the electron flux received by the layer. The layers are irradiated at increasing fluences by increasing the irradiation time. The value for the lower fluence, $\mathrm{fl}$, is $9 \times 10^{12} \mathrm{e}-/ \mathrm{cm}^{2}$ in the fl layer. The fluences $\mathrm{fO}$ in the reference $\mathrm{rf}-\mathrm{fO}$ layer and $\mathrm{fn}$ in the electron irradiated fn layers are n times f1 with $n=0,1,2,3,4$.

Layer optical characterization Absorption, PL and TRPL spectra were recorded for the reference and electron irradiated layers of the set-18 after different aging periods. In addition, the evolution of the PL spectra was recorded under continuous illumination. During the aging, the layers of the set-18 were kept in dark under flowing $\mathrm{N}_{2}$ atmosphere. The aging A0d, A20d, A53d, and A110d durations correspond to $0,20,53$, and 110 days of aging after the end of electron irradiation. Absorption, PL, and TRPL were performed after the A53d and A110d aging. For the A0d one, only absorption was recorded whereas, for the A20d one, only PL\&RTPL were recorded. Only the PL evolution under continuous illumination was recorded for the rf-17 layer in its pristine state immediately after its preparation. The delay between absorption and PL\&TRPL measurements was $+3 d$ for A53d and $+1 d$ for A110d.

Absorption Absorption spectra were recorded in air and at room temperature $(300 \mathrm{~K})$ with a double beam UV-VIS spectrophotometer (Jenway) with a wavelength resolution of $1 \mathrm{~nm}$. The incident light penetrates through the perovskite face and illuminates a rectangular spot of $\sim 5 \times 1 \mathrm{~mm}^{2}$. To span the 300-1000 $\mathrm{nm}$ range of illumination, the intensity recording versus wavelength lasts $5 \mathrm{~min}$. The incident flux is weak. A second measurement on the same layer yields to same absorption spectra than recorded during the first measurement. The (GACsMAFA) $\mathrm{Pb}(\mathrm{IBr})_{3}$ layer/glass absorbance is directly estimated from the logarithm of the ratio between the incident and transmitted light through the layer/glass structure. To this signal, to correctly estimate only the (GACsMAFA) $\mathrm{Pb}(\mathrm{IBr})_{3}$ layer absorbance, the glass absorption signal is subtracted.
The wavelength dependence of the absorbance in the HOIPs layers, $A(\lambda)$, is related to that of the transmittance by the relationship $A(\lambda)=\log _{10}((1 / t(\lambda))$. Consequently, according to the Lambert-Beer law, the absorbance is the product of the absorption coefficient $\alpha(\lambda)$ per the pathlength of the light $\mathrm{L}$ : $\mathrm{A}(\lambda)=\alpha(\lambda) \mathrm{L} / 2.303$. The transmittance, $\mathrm{t}$, is related to the reflectance, $r$, and the absorptance, $a$, in the layers via: $t=1-r-a$. The values, $A_{\exp }(\lambda)$, measured with the spectrophotometer gives $\mathrm{A}_{\exp }(\lambda)=\left(1 / \mathrm{t}_{\exp }(\lambda)\right)$ where $1=\mathrm{a}(\lambda)+\mathrm{t}_{\exp }(\lambda)$. They are consequently equal to the $A(\lambda)$ values only in the wavelength region where the scattered part of the incident light compared to the absorptance $\mathrm{a}(\lambda)$ is negligible $(\mathrm{r} / \mathrm{a}(\lambda)<<1)$. In the following, it is considered that this is a valid assumption for the investigated (GACsMAFA) $\mathrm{Pb}(\mathrm{IBr})_{3}$ HOIPs that have dull surface aspects as $\mathrm{MAPbI}_{3}$ [13].

Luminescence For the pristine $\mathrm{rf}-17$ layers and the layers set-18, the luminescence properties were first monitored by performing PL under continuous illumination. Then, for the five layers of the set-18, TRPL measurements were performed at the end of the continuous illumination after a dark period of $3 \mathrm{~min}$.

For the rf-17 (GACsMAFA) $\mathrm{Pb}(\mathrm{IBr})_{3}$ pristine layer, a $488 \mathrm{~nm}$ Coherent Sapphire laser was used at a $20 \mu \mathrm{W}$ applied power to continuously illuminate a spot diameter of $1 \mu \mathrm{m}$ in the central region of the layer. The layer was kept in air and at room temperature during illumination. The PL spectra at increasing illumination time were recorded with a wavelength resolution of $1 \mathrm{~nm}$ using an Andor spectrometer (Shamrock 303) connected to an Andor Si CCD camera (iDus Series).

For the set-18, both PL spectra under illumination and TRPL spectra were systematically recorded in the same environmental conditions as PL under illumination for rf17 , i.e., air and room temperature $(\sim 300 \mathrm{~K})$. Measurements were performed in a reflection geometry at $45^{\circ}$ using a Quantamaster 8000 spectrofluorometer equipped with a R13456 photomultiplier (Hamamatsu). To respectively conduct PL and TRPL, the spectrofluorometer was employed in two different acquisition modes: the "Steady State-Vis" mode for (steady state) PL and the "time-correlated single-photon counting (TCSPC)" one for TRPL. The PL spectra were recorded with a wavelength resolution of $1 \mathrm{~nm}$ during continuous illumination with a $454 \mathrm{~nm}$ Violet Blue Laser Diode (Cnilaser). A bandpass filter centered at $454 \mathrm{~nm}$ was used to eliminate the laser spectral wings. The laser diode delivered an applied power of $20 \mathrm{~mW}$ to illuminate a spot of about $1.5 \mathrm{~mm}$ diameter in the layer center. For TRPL, the lifetime decay spectra were recorded using a pulsed nano-LED emitting at $482 \mathrm{~nm}$. The LED emitted pulses with energy of $14 \mathrm{pJ} /$ pulse, duration of about $100 \mathrm{ps}$ with a repetition rate of $10 \mathrm{kHz}$. The laser spot has a diameter of about $250 \mu \mathrm{m}$ diameter. The emission 
signals were filtered through a high-pass filter centered at $488 \mathrm{~nm}$ before detection.

PL under illumination As regarding PL under illumination, the rf-17 pristine layer was optically excited through the perovskite face. For the set-18, the illumination was through the glass face. The reason is to investigate the regions of the perovskite layers through which sunlight in solar cells or electron beam in this work enters. In all cases, one unique spot located close to the layer center was investigated although the spot diameter was of $\sim 1 \mu \mathrm{m}$ for $\mathrm{rf}-17$ and of $\sim 1.5 \mathrm{~mm}$ for the set-18. The exact position of the spot may vary from one aging to the other one for the set-18. Consequently, one considers in the following that the spots illuminated after the A0d, A53d, and A110d aging correspond to regions near the layer centers that are different.

For the rf-17 (GACsMAFA) $\mathrm{Pb}(\mathrm{IBr})_{3}$ pristine layer, every min, the PL spectra were recorded under the $488 \mathrm{~nm}$ illumination during $300 \mathrm{~min}$. For the (GACsMAFA) $\mathrm{Pb}(\mathrm{IBr})_{3}$ layers irradiated at the different fn fluences (set-18), after the A20d, A53d, and A110d aging, the PL peak for each fluence (except $\mathrm{f} 3$ for aging 53d) was first measured after the first $3 \mathrm{~min}$ of $454 \mathrm{~nm}$ illumination and, then, every $5 \mathrm{~min}$, during two successive cycles of continuous laser illumination. Each cycle lasts $15 \mathrm{~min}$. The laser spot remains at the same position from the first to the second one. The two cycles are separated by a three mins period without illumination. The dark period is chosen of 3 min because this is the time necessary to change the spectrofluorometer configuration from the "Steady StateVis" to TCSPC mode at the end of the second cycle of illumination. This procedure allows to estimate whether, during the configuration change, the PL spectrum for which the TRPL is recorded can be expected to significantly differ from the last one recorded under illumination.

TRPL after illumination After the two cycles of continuous illumination for the A20d, A53d, and A110d and for each fluence (except $\mathrm{f} 3$ for A53d), the TRPL decays were recorded for a window range centered on the PL peak wavelength which corresponds to the first one of the second illumination cycle. At least two different spots (diameter $250 \mu \mathrm{m}$ ) were investigated for each layer of the set-18 to examine whether the PL decay lifetime spectra are homogeneous over the layer region where the PL under illumination was recorded. The TRPL measurements last from 5 to $7 \mathrm{~min}$ depending on the layer state. Consequently, before the TRPL measurement, the first spot remains under dark about $3 \mathrm{~min}$ to change the spectrometer configuration. The second ones remain a longer time under dark, for about 8 to $10 \mathrm{~min}$, depending on the recording time for the first one.

The decay lifetime spectra are recorded over a time window that is chosen to ensure that the background level is reached for each spectrum. The time resolution per channel is $56 \mathrm{~ns}$. The resolution function is within a channel. For each spot Pn, the Horiba fitting program "One-to-Four Exponentials" is used to fit the experimental decay spectra to the convolution of the sum of $\mathrm{i}(1 \leq i \leq 4)$ exponential decay components with the spectrometer resolution function and resolve the lifetime decay value $\tau_{\mathrm{iPn}}$ and their respective coefficients $\alpha_{i P n}: D(t)=\sum_{i=1}^{2} \alpha_{i P n} \exp \left(-\frac{t}{\tau_{i P n}}\right), \sum_{i} \alpha_{i P n}=1$. The average lifetime value is calculated from the fitting as: $\tau_{\mathrm{Pn}}=$ $\sum \mathrm{I}_{\mathrm{iPn}} \tau_{\mathrm{iPn}}$ with the intensity $\mathrm{I}_{\mathrm{iPn}}=\frac{\alpha_{\mathrm{iPn}} \tau_{\mathrm{iPn}}}{\sum_{j} \alpha_{\mathrm{j} n} \tau_{\mathrm{jPn}}}, \mathrm{i}=1,2$ and $\sum_{\mathrm{i}} \mathrm{I}_{\mathrm{iPn}}=1$. The lifetime data associated to each layer hereafter are the values $\tau_{\mathrm{Pn}}, \tau_{\mathrm{iPn}}$ averaged over two stops per layer as follows: $<\tau_{\mathrm{Pn}}>>_{\mathrm{Pn}}=\frac{\sum_{\mathrm{n}=1}^{2} \tau_{\mathrm{Pn}}}{2},<\tau_{1}>>_{\mathrm{Pn}}=\frac{\sum_{\mathrm{n}=1}^{2} \tau_{1 \mathrm{Pn}}}{2},<\tau_{2}>\mathrm{Pn}=\frac{\sum_{\mathrm{n}=1}^{2} \tau_{2 \mathrm{Pn}}}{2}$ and their corresponding intensity values. The standard deviations for these quantities are calculated and shown as error bars in the corresponding figures.

As a summary, Table 1 recalls the link between the layer names and states for which the optical characterizations are conducted.

\section{Results}

For the layers set-18, the absorption data are recorded over a much larger spot than those for which PL under illumination and TRPL are recorded. Furthermore, the A0d, A53d, and A110d spots for absorption were all selected at the center of the perovskite layers. The different PL and TRPL spots were also selected in the layer central region. In the following, it is considered that the absorption evolution as a function of aging duration results from the aging $\left(\mathrm{N}_{2}\right.$, dark). For the 1st PL recorded under illumination, there is the coupling of the aging $\left(\mathrm{N}_{2}\right.$, dark) with the $454 \mathrm{~nm}$ illumination lasting $3 \mathrm{~min}$ in air. For the TRPL, there is the coupling of aging $\left(\mathrm{N}_{2}\right.$, dark) with the cycles of $454 \mathrm{~nm}$ illumination in air-light (15 min); dark (3 min); light (15 min)-dark (3 min).

Aging after e-irradiation: energy dependence of absorbance This paragraph concerns the fluence-induced aging of the energy dependence of the absorbance in the (GACsMAFA) $\mathrm{Pb}(\mathrm{IBr})_{3}$ layers (set-18). For the Ajd aging, the conditions are $\mathrm{j}(\mathrm{d})=0 / 53 / 110$ under $\mathrm{N}_{2}$ atmosphere in dark at $\sim 300 \mathrm{~K}$.

As seen in Fig. 1, independently of the fluence and aging, three energy regions can be discriminated in the variation of the (GACsMAFA) $\mathrm{Pb}(\mathrm{IBr})_{3}$ reference rf-f0 and the $1 \mathrm{MeV}$ eirradiated fn layers (at fn fluence) absorbances. The mean rate of the absorbance variation per $\mathrm{eV},<\mathrm{dA} / \mathrm{dE}>$, is weak in the higher and lower energy range, i.e., for $\mathrm{E}(\mathrm{eV})>1.68$ and for $\mathrm{E}(\mathrm{eV})<1.6$, respectively. In contrast, it is quite strong for the steep step in the energy range $1.68<\mathrm{E}(\mathrm{eV})<1.6$. 
Table 1 Name, treatment, and characterization of the GA0.015Cs0.046MA0.15 2FA0.787Pb(I0.815Br0.185)3 layers spin coated on glass. The numbers 17 and 18 in the rf- 17 and set-18 names refer to the year of the preparation date of the layers

\begin{tabular}{|c|c|c|c|c|c|}
\hline Layer treatment & \multicolumn{5}{|c|}{ Pristine: as-prepared } \\
\hline Layer name & \multicolumn{5}{|l|}{$\mathrm{rf}-17$} \\
\hline Photoluminescence (PL) & \multicolumn{5}{|c|}{ PLj(488 nm, $\varphi 1 \mu \mathrm{m}, 2548 \mathrm{~W} / \mathrm{cm}^{2}$ ); on (air, $\left.300 \mathrm{~K}, 5 \mathrm{~h}\right): \mathrm{j}=1-300$} \\
\hline Layer treatment & \multicolumn{5}{|c|}{ Set-18 $\mathrm{N}_{2}$ atmosphere, dark, $300 \mathrm{~K} 7 \mathrm{~d}$ aged, e-irradiated at fluence fn, Ajd aged } \\
\hline Layer name & rf-f0 & fl & $\mathrm{f} 2$ & f3 & $\mathrm{f} 4$ \\
\hline e-Fluence (f1) & 0 & 1 & 2 & 3 & 4 \\
\hline Absorption (Abs) & \multicolumn{5}{|c|}{ Absorption air $300 \mathrm{~K}$} \\
\hline Photoluminescence (PL) & \multicolumn{5}{|c|}{$\begin{array}{l}\left.\text { PLj (454 nm, } \varphi 1.5 \mathrm{~mm}, 11 \mathrm{~W} / \mathrm{cm}^{2}\right)(\text { air, } 300 \mathrm{~K}) \\
\quad \text { on }(15 \mathrm{~min}) ; \text { off }(3 \mathrm{~min}) ; \text { on }(15 \mathrm{~min}) ; \mathrm{j}=1-6\end{array}$} \\
\hline Time-resolved PL (TRPL) & \multicolumn{5}{|c|}{ TRPL (482 nm, $\varphi 250 \mu \mathrm{m}, 14 \mathrm{pJ} / \mathrm{pulse}, 100 \mathrm{ps}, 10 \mathrm{kHz})($ air, $300 \mathrm{~K})$} \\
\hline Aging Ajd_Abs & \multicolumn{5}{|c|}{ A0d; A53d; A110d_Abs } \\
\hline Aging Ajd_PL_TRPL & \multicolumn{5}{|c|}{ A20d; A53d; A110d PL TRPL } \\
\hline
\end{tabular}

In the higher energy range, $\mathrm{E}(\mathrm{eV})>1.68$, independently of fluence, the absorbance tends to increase with increasing energy. Aging induces a strong decrease in the absorbance values. For A0d (Fig. 1a), the absorbance values reach saturation $(>4.95)$ at the following $\mathrm{E}(\mathrm{eV})$ values: $\sim 2.23$ (rf-f0, f2, f4); 2.28(f3); 2.33(f1). For A53d (Fig. 1b) and A110d (Fig. 1c), the maximum absorbance value at $\mathrm{E}(\mathrm{eV}) \sim$ $2.33 \mathrm{eV}$ is reached for the reference $\mathrm{rf}-\mathrm{f0}$ layer and the absorbance values are only 2.48 and 2.15 , respectively. Furthermore, the fluence dependence in this higher energy becomes more marked as the aging duration increases from A0d to A110d. This effect is stronger for the lower fluences. The absorbance for the $\mathrm{rf}-\mathrm{f0}$ reference layer and the irradiated f1 layer has energy dependences that are quasi-superimposed up to $2.16 \mathrm{eV}$ for A0d. They become fully separated for A53d, A110d. For the $\mathrm{f} 2$ to f4 fluences, the energy dependences for absorbance are still quasi-overlapped for the A53d aging. They start to slightly differ for the A110d aging.

In the lower energy range $\mathrm{E}(\mathrm{eV})<1.60$, the absorbance tends to decrease as energy decreases from 1.64 to $1.24 \mathrm{eV}$ for A0/53/110d. Independently on the aging duration, the absorbance values remain quite low, $\sim 0.1-0.3$ and present oscillations with higher amplitudes for the four fluences than for the rf-f0 reference layer.

The cutoff energy between the steep step $(1.68<\mathrm{E}(\mathrm{eV})<$ $1.60)$ and the lower energy region $(\mathrm{E}<1.6 \mathrm{eV})$ of the absorption spectra, as described in [14-18] and illustrated in Fig. 2, for the rf- $\mathrm{f0}$ reference layer and the irradiated $\mathrm{f} 2, \mathrm{f} 4$ layers is used to estimate the optical bandgaps. Table 2 shows that, for the longer aging duration, A110d, the optical bandgap has a slight tendency to shrink by $0.02-0.03(2) \mathrm{eV}$ with increasing fluence in the range $\mathrm{f} 2 \leq \mathrm{fn} \leq \mathrm{f} 4$.

One remark is that the fluence-induced features in the absorbance energy dependence are easier to analyze after the normalization of the absorbances to their value at $1.8 \mathrm{eV}$ (Fig. 1, inserts). For each aging duration, the normalized absorbance (Fig. 1, inserts) for each fluence overlap between 1.8 and $1.68 \mathrm{eV}$. The values in this range are super-imposed in $\mathrm{lg}$ scale on a straight line with a low slope. The steep step exhibits also a linear dependence in $\lg$ scale. As aging duration increases, clearly, three features appear: (i) the step shifts to lower energy; (ii) the fluence effect on the slope becomes

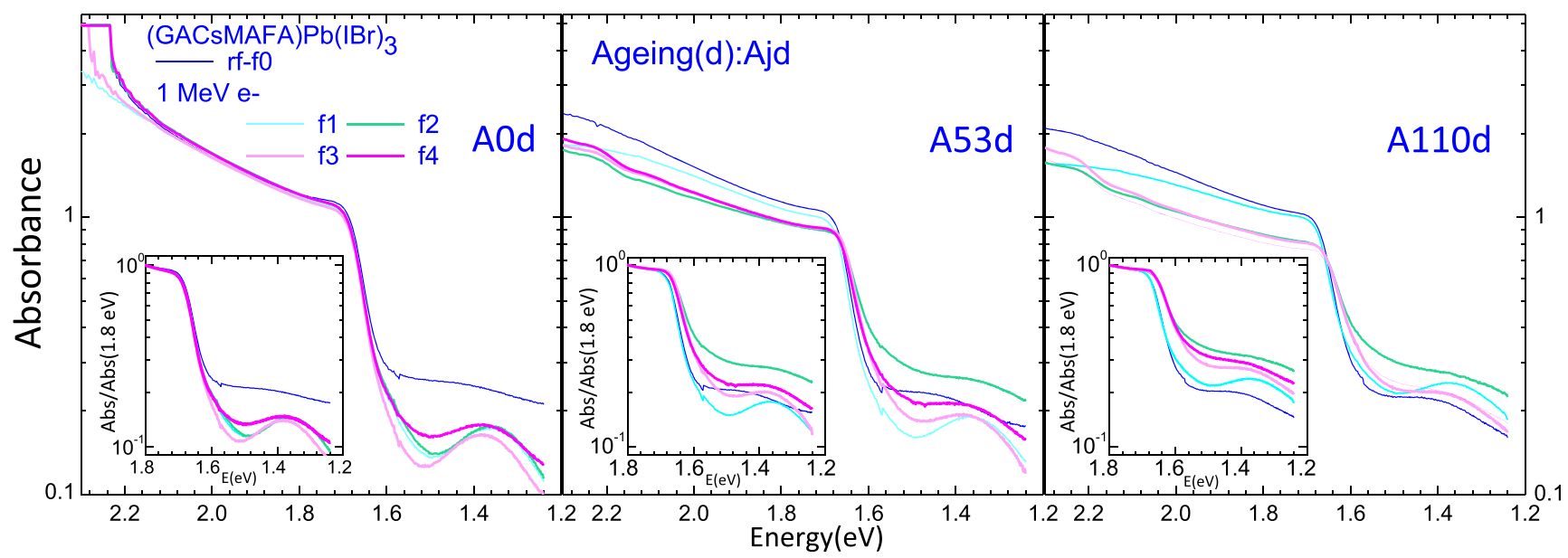

Fig. 1 Energy dependence of Absorbance (air, 300 K) after aging in (GACsMAFA) $\mathrm{Pb}(\mathrm{IBr})_{3}$ HOIPs for the reference Ajd rf-f0 layer and the $1 \mathrm{MeV}$ e-irradiated Ajd_fn layers at the fn fluence. The conditions for the
Ajd aging are $\mathrm{j}(\mathrm{d})=0 / 53 / 110$ under $\mathrm{N}_{2}$ atmosphere in dark at $\sim 300 \mathrm{~K}$. Inserts: energy dependence of absorbance (air, $\sim 300 \mathrm{~K}$ ) after normalization to the value at $1.8 \mathrm{eV}$ 


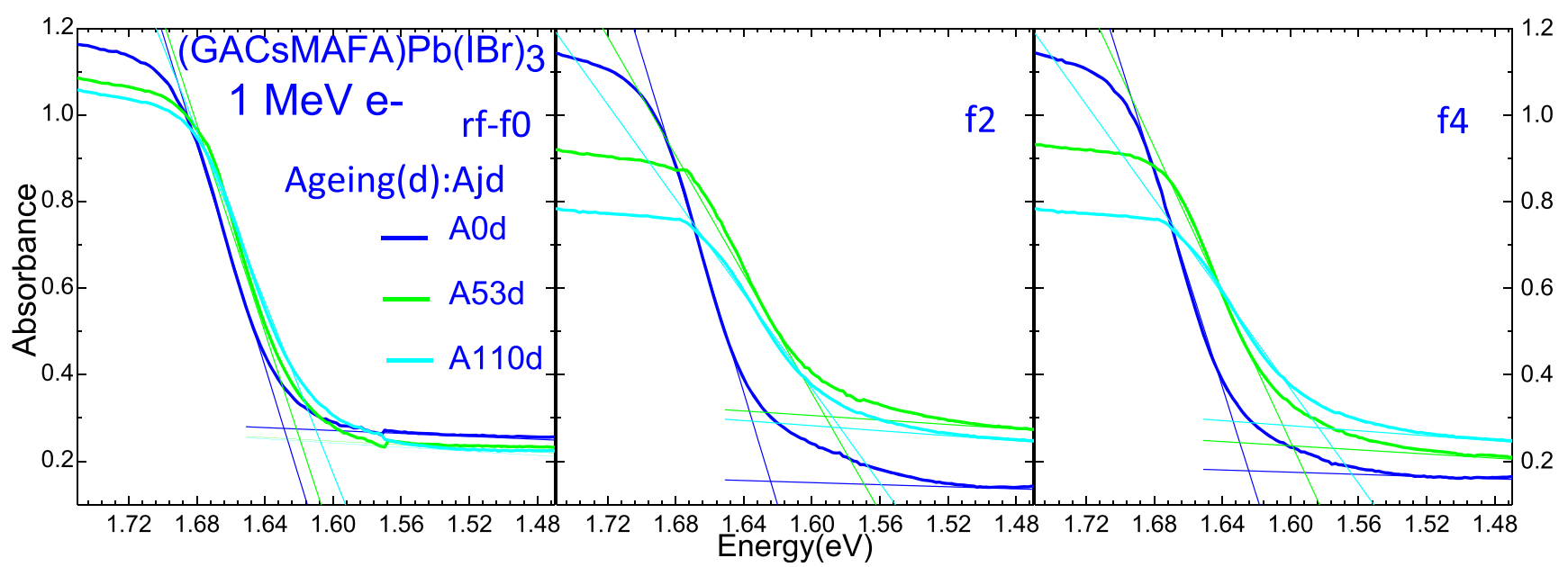

Fig. 2 Bandgap determination from the energy dependence of absorbance (air, $~ 300 \mathrm{~K}$ ) after aging in (GACsMAFA) $\mathrm{Pb}(\mathrm{IBr})_{3}$ HOIPs for the reference Ajd_rf-f0 layer and the $1 \mathrm{MeV}$-irradiated Ajd_fn layers

stronger; and (iii) the step height decreases more strongly for the higher fluences, $\mathrm{f} 2, \mathrm{f} 3, \mathrm{f} 4$ than for the lower ones, rf $\mathrm{f} 0$ (reference $\mathrm{rf}-\mathrm{f0}$ layer) and $\mathrm{fl}$. The consequence of (iii) is that the fluence-dependence of the step height evolves with increasing aging duration from $0 \mathrm{~d}(\mathrm{~A} 0 \mathrm{~d})$ to $110 \mathrm{~d}(\mathrm{~A} 110 \mathrm{~d})$. Finally, in the lower energy range below the absorption edge, $1.2 \leq \mathrm{E}(\mathrm{eV}) \leq 1.60$, a same feature is also that, independently on aging, irradiation induces stronger absorbance oscillations with a base line that in $\lg$ scale, as for the rf-f0 reference layer, tend to decrease quasi-linearly with decreasing energy.

\section{Aging after e-irradiation: fluence effect at each aging on PL} This paragraph concerns the 1 st PL peak recorded in the set- 18 of reference rf-f0 and the $1 \mathrm{MeV}$ e-irradiated fn layers (at fn fluence) after $3 \mathrm{~min}$ of $454 \mathrm{~nm}$ continuous illumination at the end of each aging, Ajd, with $j=20 / 53 / 110$. Figure 3 shows that, at each aging period, the $1 \mathrm{MeV}$ electron irradiation induces a fluence dependence for the peak energy, the peak full width at half maximum (FHWM), and intensity ratio to the PL peak intensity of the reference rf-f0 layer in its A20d_rf-f0 state. at the $\mathrm{f} 2$ and $\mathrm{f} 4$ fluence. The conditions for the Ajd aging are $\mathrm{j}(\mathrm{d})=0 / 53$ / 110 under $\mathrm{N}_{2}$ atmosphere in dark at $\sim 300 \mathrm{~K}$. The straight lines are used for the determination of the bandgap values in Table 2

First PL peak energy: fluence effect for each aging after eirradiation As seen in Fig. 3a, one easily notices that the PL peak energy at each aging has a fluence-induced distribution. The variation ranges at each aging have value in $\mathrm{eV}$, Ajd-dEp max$\min (1 \mathrm{st}): 0.020(4)(\mathrm{A} 20 \mathrm{~d}), 0.012(4)(\mathrm{A} 53 \mathrm{~d}), 0.016(4)$ (A110d). As aging duration after electron irradiation increases from A0d to A110d, this range goes through a clear minimum at A53d.

For A20d, the PL peak energy goes through a maximum at the $\mathrm{fl}$ fluence. For increasing fluence, the values in $\mathrm{eV}$ (Table 3) are respectively for A20d fn-Ep(1st): 1.585(2)(rf-f0), 1.588(2)(f1), 1.584(2)(f2), 1.582(2)(f3), and 1.568(2)(f4). With respect to $\mathrm{A} 20 \mathrm{~d}$ rf-f0-Ep(1st), the values of the peak shifts in $\mathrm{eV}$ are respectively for A20d fn-dEp(1st): 0(rf-f0), 0.003(4) (f1), 0.001(4)(f2), -0.003 (4)(f3), and $-0.0017(4)(f 4)$. The blue-shift (f1) and the redshift (f2, f3) are at the limit of detection. There is however a clear tendency for irradiation to induce a redshift increase with increasing fluence from $\mathrm{f} 2$ to $\mathrm{f} 4$.

For A53d, the PL peak energy goes through a minimum at the $\mathrm{f} 2$ fluence. The values in $\mathrm{eV}$ (Table 3 ) are respectively for A53d_fn-Ep(1st): 1.582(2)(rf-f0), 1.580(2)(f1), 1.570(2)(f2), and 1.574(2)(f4). With respect to A53d_rf-f0-Ep(1st), the values of the peak shifts in $\mathrm{eV}$ are respectively for A53d_ fn-

Table 2 Bandgaps after aging in (GACsMAFA)Pb(IBr) $)_{3}$ HOIPs for the reference Ajd-rf-f0 and the $1 \mathrm{MeV}$ e-irradiated Ajd fn layers at the fn fluence. The conditions for the Ajd aging are $\mathrm{j}(\mathrm{d})=0 / 53 / 110$ under $\mathrm{N}_{2}$ atmosphere in dark at $\sim 300 \mathrm{~K}$

\begin{tabular}{|c|c|c|c|c|c|c|c|c|c|}
\hline Ajd_fn-Eg (eV) & rf-f0 & $\mathrm{fl}$ & $\mathrm{f} 2$ & $\mathrm{f} 3$ & $\mathrm{f} 4$ & vsf0_fl & vsf0_f2 & vsf0_f3 & vsf0_f4 \\
\hline A0d & $1.63(1)$ & $1.63(1)$ & $1.63(1)$ & $1.62(1)$ & $1.63(1)$ & $-0.00(2)$ & $-0.00(2)$ & $-0.01(2)$ & $-0.00(2)$ \\
\hline A53d & $1.62(1)$ & $1.62(1)$ & $1.59(1)$ & $1.60(1)$ & $1.60(1)$ & $-0.00(2)$ & $-0.02(2)$ & $-0.02(2)$ & $-0.02(2)$ \\
\hline A110d & $1.61(1)$ & $1.61(1)$ & $1.59(1)$ & $1.58(1)$ & $1.58(1)$ & $-0.00(2)$ & $-0.02(2)$ & $-0.03(2)$ & $-0.03(2)$ \\
\hline vsA0d_A53d & $-0.01(2)$ & $-0.01(2)$ & $-0.04(2)$ & $-0.02(2)$ & $-0.03(2)$ & & & & \\
\hline vsA0d_A110d & $-0.02(2)$ & $-0.02(2)$ & $-0.04(2)$ & $-0.04(2)$ & $-0.05(2)$ & & & & \\
\hline vsA53d_A110d & $-0.01(2)$ & $-0.01(2)$ & $-0.00(2)$ & $-0.02(2)$ & $-0.02(2)$ & & & & \\
\hline
\end{tabular}




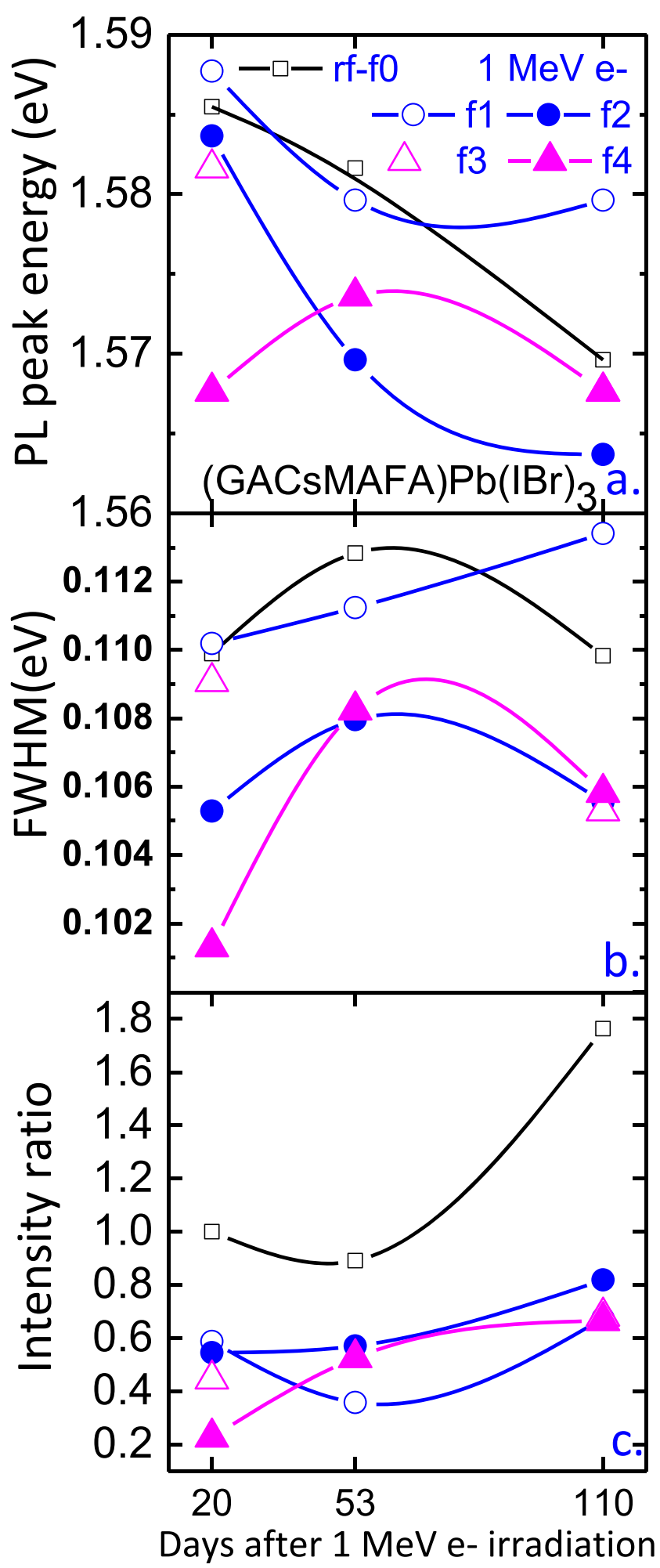

Fig. 3 Aging dependence of photoluminescence after 3-min illumination_energy peak (a), FWHM (b), intensity ratio (c) in (GACsMAFA)Pb(IBr) $)_{3}$ HOIPs for the reference Ajd rf-f0 layer and the $1 \mathrm{MeV}$ e-irradiated Ajd fn layers at the fn fluence. The conditions for the Ajd aging are $\mathrm{j}(\mathrm{d})=20 / 53 / 110$ under $\mathrm{N}_{2}$ atmosphere in dark at $\sim 300 \mathrm{~K}$. Laser Illumination is performed in (air, $\sim 300 \mathrm{~K}$ ) at the $454 \mathrm{~nm}$ wavelength and $11 \mathrm{~W} / \mathrm{cm}^{2}$ power density
$\mathrm{dEp}(1 \mathrm{st}): 0(\mathrm{rf}-\mathrm{f0}),-0.002(4)(\mathrm{f} 1),-0.012(4)(\mathrm{f} 2)$, and $0.008(4)(f 4)$. Irradiation induces a redshift at any fluence in the range $\mathrm{fl} \leq \mathrm{fn} \leq \mathrm{f} 4$. The redshift goes through a clear maximum at $\mathrm{f} 2$ fluence.

For A110d, the PL peak energy goes through a local maximum at $\mathrm{fl}$ and a local minimum at $\mathrm{f} 2$. The values in $\mathrm{eV}$ are respectively for A110d_fn-Ep(1st): $1.570(2)($ rf-f0), 1.580(2)(f1), 1.564(2)(f2), and 1.568(2) (f3,f4) (Table 4). With respect to A110d_Ep(rf-f0), values of the peak shifts in $\mathrm{eV}$ are respectively for $\mathrm{A} 110 \mathrm{~d}$ fn-dEp(1st): 0 (rf-f0), 0.010(4)(f1), $-0.006(4)(f 2)$, and $-0.002(4)(f 3, f 4)$. For A110d, irradiation induces a marked blue shift for f1. Then, as fluence increases further to $\mathrm{f} 4$, there is a redshift that goes through a maximum at $\mathrm{f} 2$.

First PL peak FHWM: fluence effect for each aging after eirradiation Figure $3 \mathrm{~b}$ shows that the FHWM at each aging has a fluence-induced distribution. The range of variation for the FHWM values goes through a minimum at A53d.

For A20d, the A20d_FHWMp_rf-f0/_fn values go through a local minimum at $\mathrm{fl}$ fluence with increasing fluence. The FWHM values in $\mathrm{eV}$ are about $0.110(4)(\mathrm{rf}-\mathrm{f0}, \mathrm{f1})$, 0.105(4)(f2), 0.109(4)(f3), and 0.101(4)(f4). At most, they differ by $-11 \mathrm{meV}$ from the higher A20d_FHWMp(rf-f0) value.

For A53d, the A53d_FHWMp_rf-f0/_fn values decrease with increasing fluence. The FWHM values in $\mathrm{eV}$ are about 0.113(4)(rf-f0), 0.111(4)(f1), and $0.108(4)(f 2, f 4)$. They differ by at most $-5 \mathrm{meV}$ from the higher value A53d_FHWMp(rf-f0).

For A110d, the A110d_FHWMp_rf-f0/_fn values go through a maximum for $\mathrm{fl}$. The FWHM values in $\mathrm{eV}$ are about 0.110(4)(rf-f0), 0.114(4)(f1), and 0.106(4)(f2, f3, f4). They differ by at most $-8 \mathrm{meV}$ from the higher A110d_FHWMp(f1) value.

First PL peak intensity: fluence effect for each aging after eirradiation At each aging, irradiation induces a fluence dependence of the PL peak intensity, Ajd_Ip_fn. One notices in Fig. $3 \mathrm{c}$ that the values of the ratios to the PL peak intensity of A20d_rf-f0, Ajd_Ip_fn/A20d-Ip_rf-f0, vary in a range that goes through a weak minimum at A53d.

For A20d, the peak intensity ratio decreases with increasing fluence: 1 (rf-f0), 0.6 (f1, f2), $0.5(\mathrm{f3}), 0.2$ (f4). The relative variation to the A20d rf-f0 peak intensity, A20d_fn-dIp(\%), tends to increase with increasing fluence: from $-40 \%$ (f1,f2) to $-80 \%$ (f4).

For A53d, the PL peak intensity ratio goes through a minimum for $\mathrm{f1}$ as fluence increases: 0.8(rf-f0), 0.4(f1), 0.6 (f2, f4),). The relative variations to the A20d-rf-f0 peak intensity, A53d fn-dIp(\%), have associated values: $-20 \%$ (rf-f0), $60 \%$ (f1), $-40 \%$ (f2, f4). It follows that A53d aging induces a decrease of the peak intensity in the A53d_rf-f0 layer of about $20 \%$. The decrease is reinforced by irradiation in the 
Table 3 First Photoluminescence peaks after aging in (GACsMAFA)Pb(IBr) $)_{3}$ HOIPs for the reference Ajd-rf-fo and the 1 MeV e-irradiated Ajd_fn layers at the fn fluence. The conditions for the Ajd aging are $\mathrm{j}(\mathrm{d})=0 / 53 / 110$ under $\mathrm{N}_{2}$ atmosphere in dark at $\sim 300 \mathrm{~K}$

\begin{tabular}{|c|c|c|c|c|c|c|c|c|c|}
\hline Ajd_fn-Ep(eV) & rf-f0 & $\mathrm{f} 1$ & $\mathrm{f} 2$ & $\mathrm{f3}$ & $\mathrm{f} 4$ & vsf0_f1 & vsf0_f2 & vsf0_f3 & vsf0_f4 \\
\hline $\mathrm{A} 20 \mathrm{~d}$ & $1.585(2)$ & $1.588(2)$ & $1.584(2)$ & $1.582(2)$ & $1.568(2)$ & $0.003(4)$ & $-0.001(4)$ & $-0.003(4)$ & $-0.017(4)$ \\
\hline A53d & $1.582(2)$ & $1.580(2)$ & $1.570(2)$ & - & $1.574(2)$ & $-0.002(4)$ & $-0.012(4)$ & - & $-0.008(4)$ \\
\hline A110d & $1.570(2)$ & $1.580(2)$ & $1.564(2)$ & $1.568(2)$ & $1.568(2)$ & $0.010(4)$ & $-0.006(4)$ & $-0.002(4)$ & $-0.002(4)$ \\
\hline vsA20d_A53d & $-0.003(4)$ & $-0.008(4)$ & $-0.014(4)$ & - & $0.006(4)$ & & & & \\
\hline vsA20d_A110d & $-0.015(4)$ & $-0.008(4)$ & $-0.020(4)$ & $-0.014(4)$ & $0.000(4)$ & & & & \\
\hline vsA53d A110d & $-0.012(4)$ & $0.000(4)$ & $-0.006(4)$ & - & $-0.006(4)$ & & & & \\
\hline
\end{tabular}

A53d fn layers more strongly at the lower fluence f1, $60 \%$, than at the higher fluences $\mathrm{f} 2 \leq \mathrm{fn} \leq \mathrm{f} 4$. The irradiation effect tends to saturate for the higher fluences $\mathrm{f} 2 \leq \mathrm{fn} \leq \mathrm{f} 4$ with the variations remaining quasi-constant, $\sim 40 \%$.

For A110d, the PL peak intensity ratio has the higher value for $\mathrm{rf}-\mathrm{f0}, \sim 1.8$, and then goes through a local maximum for $\mathrm{f} 2$ as fluence increases. The values are about 1.8 (rf-f0), 0.7 (f1), 0.9 (f2), and 0.7 (f3, f4). Compared to A20d_Ip(rf-f0), the corresponding relative variations, A110d_fn-dIp $(\%)$, are about $80 \%$ (rf-f0), $-30 \%$ (f1), $-10 \%$ (f2), and $-30 \%$ $(\mathrm{f} 3, \mathrm{f4})$. Aging at A110d induces a strong increase in the peak intensity for $\mathrm{rf}-\mathrm{f0}$ of about $40 \%$. The increase is so strongly damped by irradiation that it is totally compensated at any fluence, $\mathrm{fl} \leq \mathrm{fn} \leq \mathrm{f} 4$. The compensating effect goes through a minimum for the $\mathrm{f} 2$ fluence.

Aging after e-irradiation: fluence-induced aging of PL This paragraph concerns the aging dependence at each fluence of the 1st PL spectra in each layer of the set-18, the reference Ajd_rf-f0 layer and the Ajd_fn irradiated ones at the fn fluences. The aging behavior of the 1st PL spectra in the set18 layers couples aging in the storing conditions $\left(\mathrm{N}_{2}\right.$, dark, $300 \mathrm{~K}$ ) and $3 \mathrm{~min}$ of $454 \mathrm{~nm}$ continuous illumination (air, $300 \mathrm{~K}$ ) necessary for their recording. The aging behavior for the PL spectra for each fluence in the irradiated Ajd_fn layers can be compared to that of the Ajd_rf-f0 one to examine the synergy effects between irradiation and the Ajd agingillumination coupling. Figure 3 shows that the synergy effects strongly depend on fluence.
First PL peak energy: fluence-induced aging after e-irradiation As seen in Fig. 3a, increasing aging duration induces a fluence dependence of the peak energy in the range $\sim 1.564$ $1.588 \mathrm{eV}$. The evolutions have shapes that differ for fluences $\mathrm{f} 0$ (rf-f0 reference layer) and $\mathrm{f} 4$ from those for $\mathrm{f} 1, \mathrm{f} 2$ fluences, that are similar. Compared to A20d-Ip(fn), this results in peak shifts that evolve as aging duration increases by 33d (A53dA20d) and 90d (A110-A20d).

For the rf-f0 reference layer, there is a continuous decrease of the peak energy with increasing aging duration. The redshift for the former 33d (A53d-A20d) and the latter 47d (A110d-A53d) aging have respective values in $\mathrm{eV}$ : 0.003(4) (33d_rf-f0), -0.012(4) (47d_rf-f0). The 47d aging induces a stronger effect than the $33 \mathrm{~d}$ one and, consequently, accentuate the redshift.

With respect to the peak energy value for each fluence, A20d_Ep(fn), the (A53d-A20d)_dEp(fn) peak shifts have values in eV: $-0.014(4)\left(33 d \_f 2\right),-0.008(4)(33 d$ f1), $0.003(4)$ (33d_rf-f0), $+0.006(4)\left(33 \mathrm{~d} \_\mathrm{f} 4\right)$. This shows that the former $33 \mathrm{~d}$ aging induces redshifts for $0 \leq \mathrm{fn} \leq \mathrm{f} 2$ with a maximum for $\mathrm{f} 2$. It induces a unique blue-shift for the $\mathrm{f} 4$ fluence.

The (A110d-A20d)_dEp(fn) peak-shifts in eV are 0.020(4)(f2), - 0.015(rf-f0), - 0.014(f3), - 0.008(f1), 0(f4). It follows that aging during $90 \mathrm{~d}$ from A20d to A110d induces redshifts for $0 \leq \mathrm{fn}<\mathrm{f} 4$ and no shift for $\mathrm{f} 4$. The redshift reaches a maximum for the $\mathrm{f} 2$ fluence.

For the rf- $\mathrm{f0}$ reference layer, the aging process is accelerated as aging duration increases from $33 \mathrm{~d}$ to $47 \mathrm{~d}$. The average redshift per day increases by a factor 2.8 during the former $33 \mathrm{~d}$ to
Table 4 Urbach energies in the (GACsMAFA) $\mathrm{Pb}(\mathrm{IBr})_{3}$ HOIPs after aging in the reference Ajd-rf$\mathrm{f} 0$ and the $1 \mathrm{MeV}$ e-irradiated Ajd fn layers at the fn fluence. The conditions for the Ajd aging are $\mathrm{j}(\mathrm{d})=0 / 53 / 110$ under $\mathrm{N}_{2}$ atmosphere in dark at $\sim 300 \mathrm{~K}$

\begin{tabular}{lrrrrrrrrr}
\hline Ajd_fn-Eu (meV) & rf-f0 & \multicolumn{1}{l}{$\mathrm{f1}$} & $\mathrm{f} 2$ & $\mathrm{f} 3$ & $\mathrm{f} 4$ & vsf0_f1 & vsf0_f2 & vsf0_f3 & vsf0_f4 \\
\hline A0d & $52(2)$ & $47(2)$ & $47(2)$ & $47(2)$ & $50(2)$ & $-5(4)$ & $-5(4)$ & $-5(4)$ & $-2(4)$ \\
A53d & $53(2)$ & $50(2)$ & $83(2)$ & $61(2)$ & $67(2)$ & $-3(4)$ & $30(4)$ & $8(4)$ & $14(4)$ \\
A110d & $59(2)$ & $63(2)$ & $100(2)$ & $87(2)$ & $90(2)$ & $4(4)$ & $41(4)$ & $28(4)$ & $31(4)$ \\
vsA0d_A53d & $1(4)$ & $3(4)$ & $36(4)$ & $14(4)$ & $17(4)$ & & & & \\
vsA0d_A110d & $7(4)$ & $16(4)$ & $53(4)$ & $40(4)$ & $40(4)$ & & & \\
vsA53d_A110d & $6(4)$ & $13(4)$ & $17(4)$ & $26(4)$ & $23(4)$ & & & \\
\hline
\end{tabular}


the latter $47 \mathrm{~d}$. For $\mathrm{fl} \leq \mathrm{fn} \leq \mathrm{f} 2$, the aging process tends to slow down during the $47 \mathrm{~d}$ aging with the redshift per day decreasing by a factor of 0.3 (f2) or 0 (f1). For the f4 fluence, the $33 \mathrm{~d}$ and $47 \mathrm{~d}$ aging have opposite effects with the result that the later $47 \mathrm{~d}$ aging compensates the changes induced during the former $33 \mathrm{~d}$ aging. The redshift rate per day is slower by a factor of 0.7 for the $47 \mathrm{~d}$ aging than for the $33 \mathrm{~d}$ one.

First PL peak FHWM: fluence-induced aging after e-irradiation As seen in Fig. 3b, the aging-dependent values for the peak FWHM are in the same range for fluences $\mathrm{f} 0$ (rf-f0 reference layer), $\mathrm{f} 1$ and in lower ones for fluences $\mathrm{f} 2, \mathrm{f} 3$, $\mathrm{f} 4$ : 0.1010.108 (f2, f4), $\sim 0.106-0.110$ (f3), 0.110-0.113 (rf-f0, f1).

The aging evolution of the PL peak FHWM has a similar shape for the $\mathrm{rf}-\mathrm{f} 0$ reference layer and the $\mathrm{f} 2, \mathrm{f} 4$ irradiated ones with a maximum at A53d. This maximum is clearly absent for the f1 irradiated layer where the FWHM continuously increases with increasing aging duration. It follows that aging for $\mathrm{rf}-\mathrm{f} 0, \mathrm{f} 2$, f4 layers has opposite effects during the former 33d period (A53d-A20d) and the latter 47d period (A110dA53d) on the FHWM behavior.

The A20d-FWHM_rf-f0/_fn values are lower for the $\mathrm{f} 4$ layer than for the rf-f0, f2 layers. The A110d-FWHM_rf-f0/ fn values are quasi the same with respect to the A20dFWHM_rf-f0/_fn values for $\mathrm{rf}-\mathrm{f0}, \mathrm{f} 2$ layers. This results in a steeper maximum for the $\mathrm{f} 4$ layer than for the rf- $\mathrm{f} 0$, $\mathrm{f} 2$ layers.

Compared to the reference rf-f0 layer, irradiation has consequently effects on the peak FWHM aging that appears to be stronger for the layers $\mathrm{f} 1, \mathrm{f} 4$ than for the $\mathrm{f} 2$ layer.

First PL peak intensity: fluence-induced aging after e-irradiation As seen in Fig. 3c, the evolution of the PL peak intensity ratio, Ajd_Ip_rf-f0/_fn/A20d_Ip_rf-f0, has a similar shape for the rf-f0 reference layer and irradiated $\mathrm{fl}$ layer with a minimum value at $A 53 \mathrm{~d}$. The behaviors for the irradiated $\mathrm{f} 2$ and $\mathrm{f} 4$ layers are respectively different from those. The peak ratio for the $\mathrm{f} 2$ layer remains quasi-constant from A20d to A53d and then increases. There is a continuous increase for the $f 4$ layer that tends to saturate between A53d and A110d. These features show that there is a fluence threshold $\mathrm{f} 1<\mathrm{fn}<\mathrm{f} 2$ above which the aging effects become clearly different in the irradiated layers from those in the reference rf-f0 one. Furthermore, for any aging duration, the higher peak intensity ratios are for the rf-f0 layer. The ratios Ajd_Ip_rf-f0/_fn /A20d_Ip_rf-f0 are below 1 except for rf-f0 at A110d. Consequently, the irradiation-aging induces a decrease of the PL peak intensities for any fluence $\mathrm{fl} \leq \mathrm{fn} \leq \mathrm{f} 4$. When compared to A20d Ip(rf-f0), the coupling between aging and irradiation has the stronger effect for A20d Ip(f4), approx. $-80 \%$, and the lower one for A110d_Ip(f2) approx. $-20 \%$.
Illumination-time dependence of PL: pristine layer Figure 4 a shows that 488-nm illumination at high intensity in air has a strong effect on the PL spectra in the pristine rf-17 layer. As illumination time increases from 1 to $300 \mathrm{~min}$, the differences between the last 300th and 1st PL spectra for the peak (i) energy, (ii) FWHM and (iii) intensity are respectively in $\mathrm{eV}$ and $\%$ : rf-17(i)-dEp ${ }_{\sim 300 K}(300$ th-1st)- (-0.058(1)); (ii)-FHWM $\sim 300 \mathrm{~K}$ (300th-1st)- 0.100(1); (iii)-dIp $\sim 300 \mathrm{~K}$ (300th-1st)- (-100). The detailed examination below shows that the stronger variations are during the $1 \mathrm{st} \mathrm{hr}$. of illumination for (i) and (iii) (Fig. $4 \mathrm{~b}, \mathrm{~d}$ ) and during the last 5 th $\mathrm{hr}$ for (ii) (Fig. 4c).

rf-17_PL peak energy under continuous illumination The decrease of the PL peak energy during illumination can be separated in four stages that roughly begin and end at the following time (min): (i) 1-8; (ii) $10-18$; (iii) $20-60$ for the 1 st hour; (iv) $240-300$ for the 5 th hour.

The (i) $1-8$ stage (i.e., between 1 and $8 \mathrm{~min}$ ) is the steeper one where the peak energy decreases during the first $8 \mathrm{~min}$ of illumination from 1.6426(5) to $1.6047(5) \mathrm{eV}$ with an average decrease rate of approx. less than $-4.74 \times 10^{-3}>\mathrm{eV} / \mathrm{min}$. During this stage, the redshift is the higher for the first $2 \mathrm{~min}$ of illumination with a decrease rate, $-1.804 \times 10^{-2} \mathrm{eV} / \mathrm{min}$, about 3 times higher than the average one.

Then, there is the (ii) 10-18 stage I,a transitory quasisteady state where the PL peak energy levels off at $1.6058(1) \mathrm{eV}$.

During the (iii) 20-60 stage, the PL peak energy slowly decreases, les than $-1.44 \times 10^{-4}>\mathrm{eV} / \mathrm{min}$ and reaches $1.5921(5) \mathrm{eV}$.

The (iv) 240-300 stage corresponds to a quasi-steady state with fluctuations in the range 1.5875(5)-1.5818(5) $\mathrm{eV}$ that slowly tend to decrease the peak energy at a rate of less than $-3.81 \times 10^{-5}>\mathrm{eV} / \mathrm{min}$ from $1.5863(5)$ to $1.5852(5)$.

rf-17_PL peak FWHM under continuous illumination The rf17_FHWM has a simple evolution (Fig. 4c). From the 1st to the last 5th hour, there is a continuous increase. During the 1st hour, the rf-17_FHWM slowly increases with an average rate of $<3.17 \times 10^{-4}>\mathrm{eV} / \mathrm{min}$. Its values increase from $0.099(1)$ to $0.118(1) \mathrm{eV}$. During the 5th hour, the rf-17_FWHM increases from $0.161(1)$ to 0.199 (1) (1st) with a faster rate of $<6.33 \times 10^{-4}>\mathrm{eV} / \mathrm{min}$.

rf-17_PL peak intensity under continuous illumination The evolution of the peak intensity in Fig. $4 d$ can be separated in four stages that roughly begin and end at the following time (min): (i) $1-8$; (ii) $10-18$; (iii) $20-60$ for the 1st hour; (iv) 240-300 for the 5th hour. These stages correspond to successive slowing-downs of the rf-17 Ip decrease rate $(\% / \mathrm{min})$ : (i) $<-9.00>$; (ii) $<-$ $1.25>$; (iii) $<-2.56 \times 10^{-1}>$; (iv) $<-7.32 \times 10^{-3}>$. During the 1 st stage (i), the decrease is the highest 


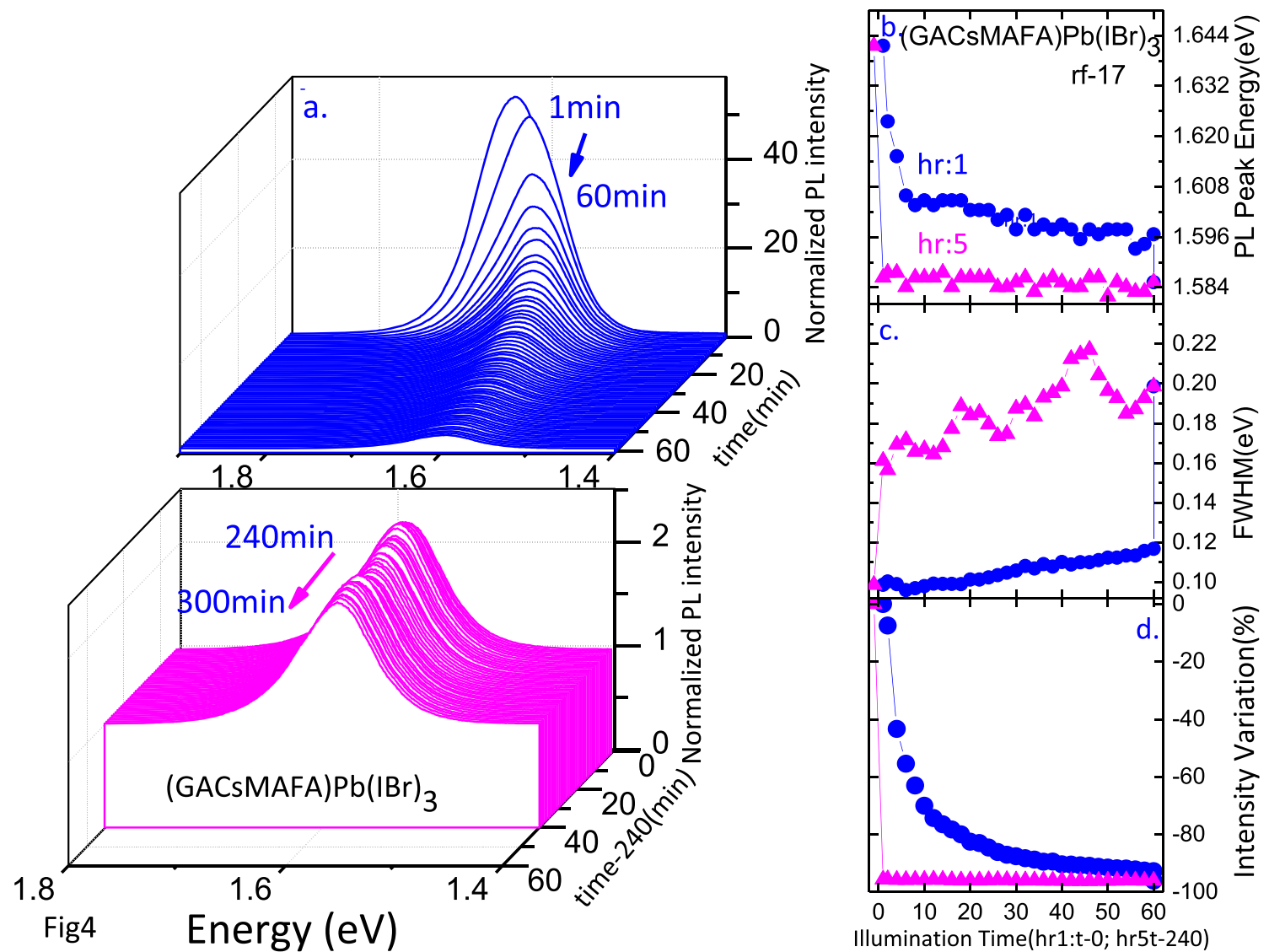

Fig. 4 Illumination-time dependence of photoluminescence intensity normalized to the background (a), energy peak (b), FWHM (c), intensity ratio (d) after aging in (GACsMAFA) $\mathrm{Pb}(\mathrm{IBr})_{3} \mathrm{HOIPs}$ for the

pristine rf-17. Laser illumination is performed in (air, $\sim 300 \mathrm{~K}$ ) at the $488 \mathrm{~nm}$ wavelength and $2548 \mathrm{~W} / \mathrm{cm}^{2}$ power density

during the first 2 and 4 min of illumination with a decrease rate of $-17.9 \% / \mathrm{min}$. The peak intensity decreases from 54.8 to $3.98 \%$ during the first hour and from 2.41 to $2.17 \%$ during the last hour.

Aging after electron irradiation: fluence effect on PL illumination-time dependence In the set-18 (reference Ajd_rf-f0 layer and the Ajd_fn irradiated ones at fn fluence), for each aging duration $\operatorname{Ajd}(\mathrm{j}(\mathrm{d})=20,53,110)$, the evolution of the PL spectra during the $454 \mathrm{~nm}$ illumination cycles depends on the electron irradiation fluence (Fig. 5). Furthermore, one notices that the evolution of the 4th-5th-6th spectra during the second cycle of 15-min illumination, is generally consistent with that of the 1st-2nd-3rd spectra during the first cycle of $15 \mathrm{~min}$ illumination. The dark period of $3 \mathrm{~min}$ between the two cycles of 15-min continuous illumination stops or induces a slight recovery of the PL evolution in most cases.

PL peak energy: fluence effect on the aging of its illumination-time dependence After each aging period, the peak energy in Fig. 5a-e has illumination time-dependence that varies with fluence. The decrease is generally steeper during the first illumination cycle. It tends to level off than

during the second one. The most striking effect of fluence on the peak energy of each ${ }^{\text {th }}$ PL spectra recorded during illumination is that, at each aging, the fluence f1 plays a unique role. Independently on the aging period, this is the fluence at which each $n^{\text {th }}$ PL peak energy goes through a maximum under illumination. These values, Ajd_f1-Ep( $\left({ }^{\text {th }}\right)$, in $\mathrm{eV}$ are higher for A20d than for A53d and, only slightly, for A53d than for A110d.

For A20d, the $\mathrm{n}^{\text {th }}$ PL spectra with $4 \leq n \leq 6$ spectra recorded during the second illumination cycle exhibit one peak. The $\mathrm{n}^{\text {th }}$ peak energy varies in a fluence-dependent range such that it increases with fluence as follows: A20d_f4-Ep $\left(\mathrm{n}^{\text {th }}\right)<$ f2$E \mathrm{E}\left(\mathrm{n}^{\text {th }}\right)<\_$f3-Ep $\left(\mathrm{n}^{\text {th }}\right)<$ rf-f0-Ep $\left(\mathrm{n}^{\text {th }}\right)<\_$f1-Ep $\left(\mathrm{n}^{\text {th }}\right)$. In respect to A20d_(rf-f0, fn)-Ep(1st), the redshifts for the 6th spectra recorded at the end of the illumination cycles, A20d_(rf-f0, fn)-dEp(6th) goes through a maximum for the f2 fluence where it reaches $-0.017(4) \mathrm{eV}$. The redshift is the smaller for the $\mathrm{fl}$ fluence, at the limit of detection. These values are, in eV, A20d_(rf-f0, fn)-dEp(6th): - 0.007(4)(rf-f0), 0.004(4)(f1), $-0.017(4)(\mathrm{f} 2),-0.014(4)(\mathrm{f} 3),-0.010(4)(\mathrm{f} 4)$.

For A53d, the $\mathrm{n}^{\text {th }}$ PL spectra with $4 \leq n \leq 6$ spectra recorded during the second illumination cycle exhibit one peak. The $\mathrm{n}^{\text {th }}$ peak energy varies in a fluence-dependent range such that it 


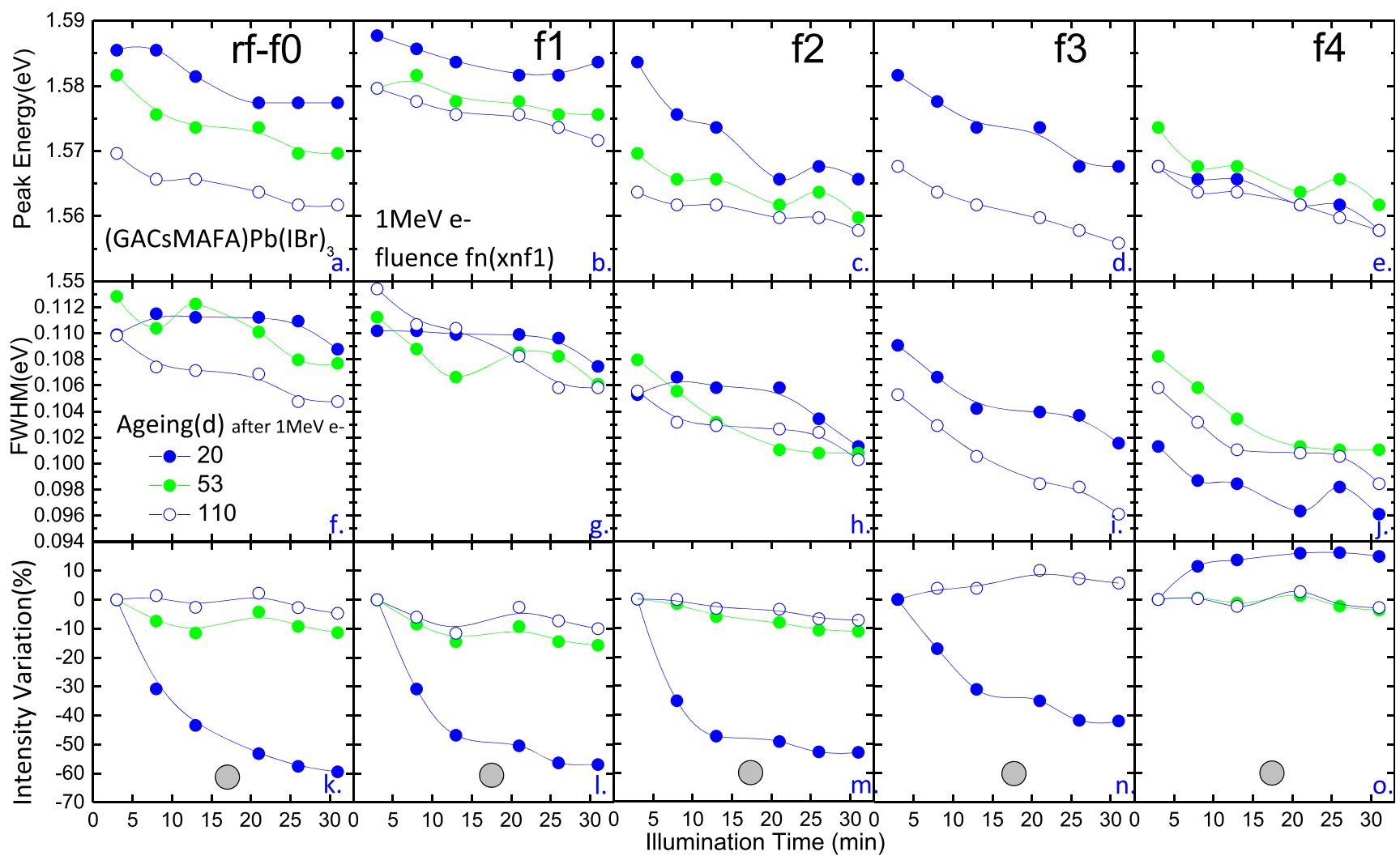

Fig. 5 Illumination-time dependence of photoluminescence_energy peak $(\mathrm{a}-\mathrm{e})$, FWHM ( $\mathrm{f}-1)$, intensity ratio $(\mathrm{m}-\mathrm{q})$ after aging in (GACsMAFA) $\mathrm{Pb}(\mathrm{IBr})_{3}$ HOIPs for the Ajd rf-f0 reference layer and the Ajd fn $1 \mathrm{MeVe}$-irradiated layers at the fn fluence. The conditions for the Ajd aging are $\mathrm{j}(\mathrm{d})=20 / 53 / 110$ under $\mathrm{N}_{2}$ atmosphere in dark at $\sim 300 \mathrm{~K}$.
Laser illumination is performed in (air, $300 \mathrm{~K}$ ) at the $454 \mathrm{~nm}$ wavelength and $11 \mathrm{~W} / \mathrm{cm}^{2}$ power density. Two cycles of $15 \mathrm{~min}$ of illumination are separated by a dark period of $3 \mathrm{~min}$ : light (15 min); dark ( $3 \mathrm{~min})$; light (15 $\mathrm{min})$ increases with fluence as follows: A53d $\mathrm{f} 2-\mathrm{Ep}\left(\mathrm{n}^{\text {th }}\right)<\mathrm{f} 4-$ $\operatorname{Ep}\left(\mathrm{n}^{\text {th }}\right)<\mathrm{rf}-\mathrm{f0}-\mathrm{Ep}\left(\mathrm{n}^{\text {th }}\right)<\mathrm{fl}-\mathrm{Ep}\left(\mathrm{n}^{\text {th }}\right)$. In respect to A53 $\overline{\mathrm{d}} \mathrm{rf}-$ $\mathrm{fO} / \mathrm{fn}-\operatorname{Ep}(1 \mathrm{st})$, the redshifts for the 6 th spectra recorded at the end of the illumination cycles, A53d rf-f0/ fn- $\mathrm{dEp}(6 \mathrm{th})$, is maximum for the $\mathrm{f} 0$ (reference $\mathrm{rf}-\mathrm{f0}$ layer), $\mathrm{f} 4$ fluences where it reaches $-0.012(4) \mathrm{eV}$. The redshift is the smaller for the $\mathrm{fl}$ fluence. These values are, in eV, A53d rf-f0/ fn-dEp(6th): 0.012(4)(rf-f0), -0.004(4)(f1), - 0.010(4)(f2), - 0.012(4)(f4).

For A110d, the $\mathrm{n}^{\text {th }}$ PL spectra with $4 \leq n \leq 6$ spectra recorded during the second illumination cycle exhibit one peak. The $\mathrm{n}^{\text {th }}$ peak energy varies in a fluence-dependent range such that it increases with fluence as follows: A110d_f3-Ep $\left(\mathrm{n}^{\text {th }}\right)<$ f4$\operatorname{Ep}\left(\mathrm{n}^{\text {th }}\right) \sim$ f2-Ep $\left(\mathrm{n}^{\text {th }}\right)<\_$rf-f0-Ep $\left(\mathrm{n}^{\text {th }}\right)<\_$f1-Ep $\left(\mathrm{n}^{\text {th }}\right)$. With respect to A110d_rf-f0/ fn-Ep(1st), the redshifts for the 6 th spectra recorded at the end of the illumination cycles, A110d_rf-f0/_fn-dEp(6th) goes through a maximum for the fluence $\mathrm{f3}$ where it reaches $-0.012(4) \mathrm{eV}$. They are quasiequal for the fluences $\mathrm{f0}$ (reference rf-f0 layer), f1, f2, f4, approx. $-0.010(4) \mathrm{eV}$.

PL peak FWHM: fluence effect on the aging of its illuminationtime dependence After each aging period, the PL peak FWHM in Fig. $5 f-j$ has illumination-time dependence that varies with fluence. The most striking effect of fluence on the peak FHWM is that, at any aging, the $\mathrm{f} 2$ fluence appears as a threshold value for which, for $\mathrm{fn} \geq \mathrm{f} 2$, there is a clear narrowing of the $\mathrm{n}^{\text {th }}$ $(1 \leq n \leq 4)$ spectra. The FHWM values for $\mathrm{fn} \leq \mathrm{fl}$, Ajd $\mathrm{rf}-\mathrm{f0} / \mathrm{fn}-$ FWHM $\left(\mathrm{n}^{\text {th }}\right)$, are $\geq 0.106 \mathrm{eV}$. The FHWM values for $\mathrm{fn} \geq \mathrm{f} 2$, Ajd_rf-f0/_fn-FWHM(n $\left.{ }^{\text {th }}\right)$, are $\leq 0.106 \mathrm{eV}$.

The FHWM values for each aging and fluence are always lower for the last spectra (6th) than for the first spectra recorded under illumination: Ajd_rf-f0/_fnFWHM(6th)<Ajd_rf-f0/_fn-FWHM(1th). The spectra narrowing depends on aging and fluence.

For A20d, the FWHM values in eV for the 6th spectra decrease with increasing fluence: A20d_rf-f0-FWHM(6th): $0.108(4) \sim$ f1-FWHM(6th): 0.108(4)>ff-FWHM(6th): $0.101(4) \sim$ f3-FWHM(6th): 0.101>ff4-FWHM(6th): 0.096(4). For A53d, the narrowing become quasi-equal. A110d, the narrowing goes through a minimum for the $\mathrm{f} 3$ fluence. The lower value for Ajd_fn-FWHM(6th), $0.096(4) \mathrm{eV}$, is reached in two cases: A20d_f4-FWHM(6th); A110d_f3-FWHM(6th). 
For each aging, the illumination-time dependence exhibits slight fluence dependent differences. The differences vary with the illumination cycle. The aging has different effects on the fluence dependence of each cycle. As concern the 1st to 3 rd spectra recorded during the first illumination cycle, the main effect of aging is to increase the fluence range where the Ajd_rf-f0/ fn-FWHM $\left(\mathrm{n}^{\text {th }}\right)(1 \leq n \leq 3)$ values decrease with increasing illumination time. For A20d, a decrease of the A20d fn-FWHM( $\left.\mathrm{n}^{\text {th }}\right)(1 \leq n \leq 3)$ values is observed only for $\mathrm{fn} \geq \mathrm{f} 3$. For A53d, it already occurs for $\mathrm{fn} \geq \mathrm{f1}$. For A110d, it is already present in the $\mathrm{rf}-\mathrm{f} 0$ reference layer. As concern the 4th to 6th spectra recorded during the second illumination cycle, the main aging effect is for the fluence $\mathrm{f} 2$. As aging increases from A20d to A110d, clearly the illumination dependence for Ajd_f2-FHWM $\left({ }^{\text {th }}\right)(4 \leq n \leq 6)$ is reduced: from decreasing for A20d to nearly flat for A53d, A110d.

PL peak intensity: fluence effect on the aging of its illumination-time dependence After each aging period, the PL peak intensity in Fig. 5k-o has illumination-time dependence that varies with fluence. For each aging, the shape changes suddenly with increasing fluence. The threshold fluence for these changes are A20d-f4, A53d-f3, and A110d$\mathrm{f} 4$, respectively. The detailed analysis below shows that $1 \mathrm{MeV}$ irradiation progressively affects the $454 \mathrm{~nm}$ illumination effect as fluence increases from $\mathrm{fl}$ to $\mathrm{f} 4$. Increasing the fluence irradiation dampens the illumination effect more strongly for the shorter aging duration, A20d, than for the longer ones, A53d, A110d. The damping effect is the higher at the $\mathrm{f} 4$ fluence for A20d. It goes through a maximum at $\mathrm{f3}$ for A110d.

For A20d, the illumination-time dependence is different for the lower fluence range, $\mathrm{fn} \leq \mathrm{f}$, and the higher one, $\mathrm{f3}<\mathrm{fn} \leq$ $\mathrm{f} 4$. For $\mathrm{fn} \leq \mathrm{f} 3$, the 454 illumination induces a steep decrease with increasing illumination time for the spectra $(1 \leq n \leq 3)$ recorded during the first cycle. The decrease tends to level off during the second illumination cycle for the spectra $(4 \leq$ $n \leq 6$ ). For the fluence $f 4$, illumination with time induces an increase of the peak intensity that tends to level off during the first and second illumination cycles for the spectra $1 \leq n \leq 6$. Compared to the first peak $(n=1)$, the relative variation for the last peak, A20d_fn-dI(6th) progressively increase with increasing fluence: $-62 \%(\mathrm{rf}-\mathrm{f} 0)<-53 \%(\mathrm{f1})<-50 \%$ (f2) $<-$ $40 \%$ (f3) $<+13 \%$ (f4).

For A53d, A110d, the illumination-time dependence has two type of shapes depending whether the fluence is in the lower range, $\mathrm{fn} \leq \mathrm{f} 2$, or the higher one $\mathrm{f} 2<\mathrm{fn} \leq \mathrm{f} 4$. For $\mathrm{fn} \leq \mathrm{f} 2$. The $454 \mathrm{~nm}$ illumination with time induces a small decrease in the peak intensity, $-20 \leq \mathrm{A} 20 \_\mathrm{dIp}(\%) \leq \sim 0$, during the first and second cycles for the spectra $1 \leq n \leq 6$. For the fluence range, $\mathrm{f} 2<\mathrm{fn} \leq \mathrm{f} 4$, the peak intensity tends to remain constant. For all layers (rf-f0 and fn irradiated ones), the dark period of 3 min seems to induce a small increase of the peak intensity from the 4 th to the 5 th spectrum, $0 \leq \mathrm{A} 20$ dIp-5th-4th $(\%) \leq$ $\sim+5$, between the two cycles.

Aging after electron irradiation: fluence-induced aging dependence of PL decay The comparison of the experimental and fitted PL lifetime decay spectra versus increasing time in Fig. 6, Ajd_fn-TRPL(t), for the fluences $\mathrm{f1}, \mathrm{f} 4$ and $\mathrm{j}(\mathrm{d})=20$, 53,110 , illustrates that the increase of aging duration, from $20 \mathrm{~d}(\mathrm{~A} 20 \mathrm{~d})$ to $90 \mathrm{~d}(\mathrm{~A} 110 \mathrm{~d}$ ), tends to reduce the small difference between the time-dependence recorded over each spot per layer. This tendency is true for any fluence $\mathrm{f} 0$ (reference rff0 layer) $\leq \mathrm{fn} \leq \mathrm{f} 4$, which suggests that the layers tend to become more homogeneous as aging duration increases. Figure 6 also illustrates that the decay slopes, Ajd_fnTRPL $(t)$, vary differently with increasing aging. For A20/ 53d, the backgrounds Ajd_fn-TRPL-BKL $(\mathrm{t})$ are indeed higher for $\mathrm{f} 4$ than $\mathrm{f1}$. For A110d, this is the opposite for the A110d_fn-TRPL-BKL(t) that is lower for $\mathrm{f} 4$ than $\mathrm{fl}$. This aging effect is reflected in the fluence-dependences of the spectra average lifetime, Ajd_ $<\tau_{\mathrm{Pn}}>_{\mathrm{Pn} \_} \mathrm{rf}-\mathrm{fO} /$ fn and those of the two Ajd_fn_ $\left.\left\langle\tau_{\mathrm{i}}\right\rangle_{\mathrm{Pn}} \mathrm{rf}-\mathrm{f0} / \mathrm{fn}, \mathrm{Ajd} \mathrm{fn}_{-}<\mathrm{I}_{\mathrm{i}}\right\rangle_{\mathrm{Pn}} \mathrm{rf}-\mathrm{f0} / \mathrm{fn}$ spectra components as seen in Fig. 7. The average lifetimes (Fig. 7a) go through a weak minimum, $\mathrm{Ajd}_{-}<\tau_{\mathrm{Pn}}>_{\mathrm{Pn} \_} \mathrm{rf}-\mathrm{fO} / \mathrm{fn}$ min, that is located in different fluence ranges depending on aging and value in ns that increases with aging as follows: A20d_f $\leq \mathrm{fn} \leq \mathrm{f3}-1440(10)$; A53d_f1-1933(69); A110d $\mathrm{f} 2 \leq \overline{\mathrm{fn}} \leq \mathrm{f} 3-2410(50)$. The maximum values, $\mathrm{f}$ $\mathrm{Ajd} \_<\tau_{\mathrm{Pn}}>\mathrm{Pn} \_$rf-f0/_fn max are reached at fluences that decrease with increasing aging and have values in ns that go through a minimum with increasing aging: A20d_f44435(600); A53d_rf-f0-3004(466); A110d_rf-f0-4275(300).

The lifetime components (Fig. 7b, c), Ajd_fn_ $<\tau_{\mathrm{i}}>\mathrm{Pn}_{-} \mathrm{rf}-\mathrm{fO} /$ _fn, with increasing fluence have shapes quite similar to those of Ajd_ $\left.<\tau_{\mathrm{Pn}}\right\rangle_{\mathrm{Pn} \_} \mathrm{rf}-\mathrm{fO} / \mathrm{fn}$. The minima, Ajd_fn_ $\left\langle\tau_{\mathrm{i}}\right\rangle \mathrm{Pn}_{-} \mathrm{rf}-\mathrm{fO} /$ fn $\min$, in ns are for the lower and higher component, respectively:

Ajd_fn $-<\tau_{1}>$ Pn_rf-f0/_fn min

: A20d_f2 $\leq \mathrm{fn} \leq \mathrm{f3} ;$ A53d_f1; A110d_f2 $\leq \mathrm{fn} \leq \mathrm{f3}$

Ajd_fn_ $<\tau_{2}>$ Pn_rf-f0/_fn min

\section{: A20d_f2 $\leq \mathrm{fn} \leq \mathrm{f} 3 ;$ A53d_f1; A110d_f2 $\leq \mathrm{fn} \leq \mathrm{f3}$}

The maxima Ajd_fn_ $\left.<\tau_{\mathrm{i}}\right\rangle_{\mathrm{Pn} \_}$rf- $\mathrm{f0} /$ fn max are reached at fluences that decrease with increasing aging and have values in ns that go through a minimum with increasing aging:

Ajd_fn_ $<\tau_{1}>$ Pn_rf-f0/_fn max

: A20d_f4;A53d_f0;A110d_rf-f0

Ajd_fn $\_<\tau_{2}>$ Pn_rf-f0/_fn max

: A20d_f4;A53d_f0;A110d_rf-f0 
Fig. 6 Time-resolved photoluminescence recorded after 30 min laser illumination (Fig. 5) over two Pn_Ajd spots after aging in (GACsMAFA) $\mathrm{Pb}(\mathrm{IBr})_{3}$ HOIPs for the $1 \mathrm{MeV}$ e-irradiated Ajd_fn layers at the $\mathrm{fl}$ and $\mathrm{f} 4$ fluences.

The conditions for the Ajd aging are $\mathrm{j}(\mathrm{d})=20 / 53 / 110$ under $\mathrm{N}_{2}$ atmosphere in dark at $\sim 300 \mathrm{~K}$. The TRPL spectra are recorded using a nano-LED_482nm, $14 \mathrm{pJ} / \mathrm{pulse}$ energy, 100 ps pulse duration, and $10 \mathrm{kHz}$ repetition rate. The laser spot has a diameter of about $250 \mu \mathrm{m}$ diameter. Straight lines are fits to the experimental decays

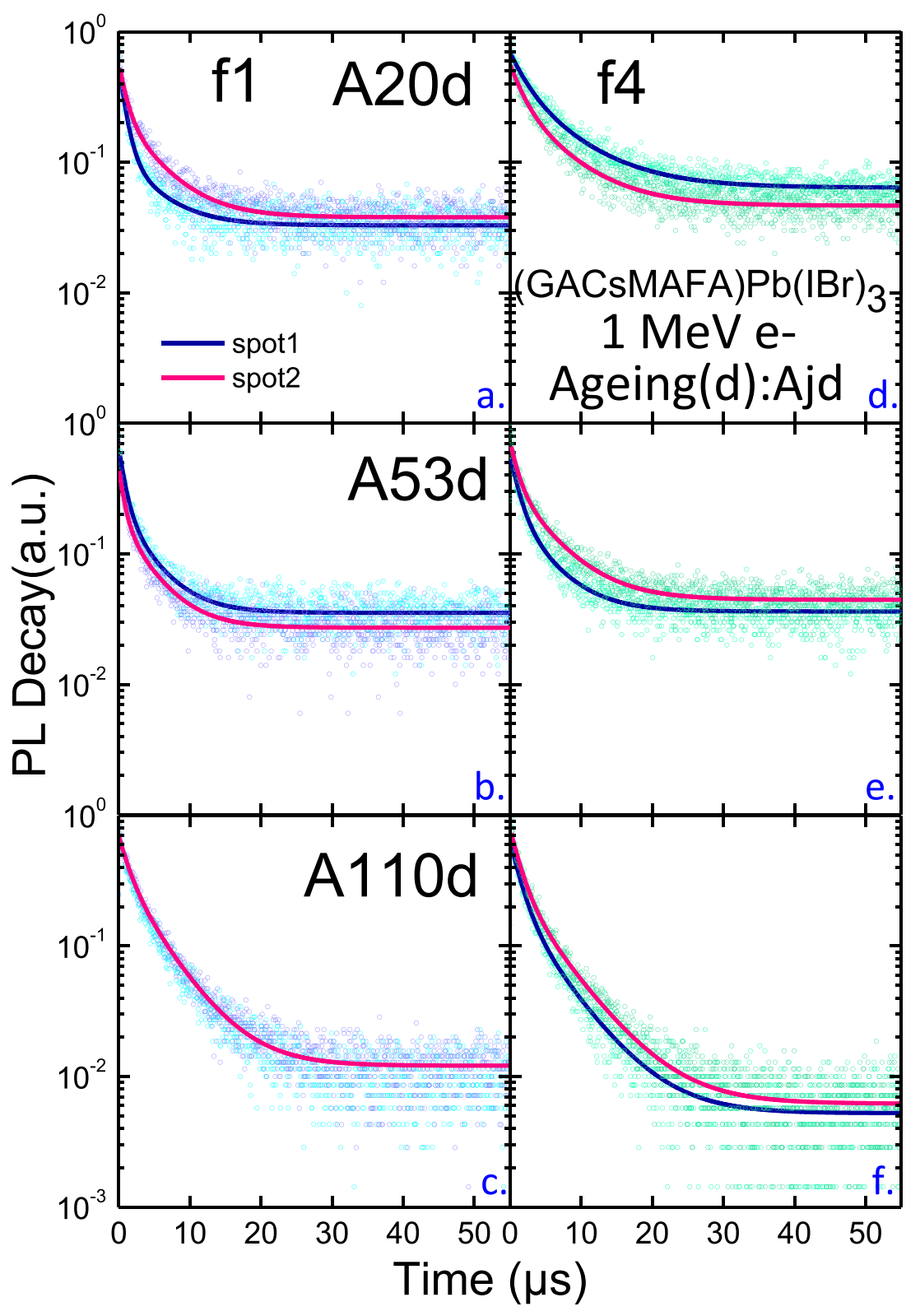

The intensities for the lower component (Fig. 7d), Ajd_ $<\mathrm{I}_{1}>_{\mathrm{Pn}} \mathrm{rf}-\mathrm{fO} / \mathrm{fn}$, with increasing fluence go through two local minima at $\mathrm{f} 2, \mathrm{f} 4$ for A20d and through a maximum at $\mathrm{f} 2$ for $\mathrm{A} 53 / 110 \mathrm{~d}$. The values in $\%$ vary in the range $50<$ Ajd_ $<\mathrm{I}_{1}>_{\text {Pn_rf-fO/_fn }}<75 \%$.

The contribution of each component, $\mathrm{Ajd} \mathrm{C}_{-}<\mathrm{I}_{\mathrm{i}} \tau_{\mathrm{i}}>\mathrm{rf}-\mathrm{fO} / \mathrm{fn}$ to the average lifetime vary with increasing fluence and aging. The stronger contribution ( $>50 \%)$ is at any fluence and aging from the longer component. This reflects that the variation of the lifetime Ajd $\left.<\tau_{2}\right\rangle_{\mathrm{Pn} \_} \mathrm{rf}-\mathrm{f0} / \mathrm{fn}$, compensates the variation of its intensity $\mathrm{Ajd} \_<\mathrm{I}_{2}>_{\mathrm{Pn} \_} \mathrm{rf}-\mathrm{fO} / \mathrm{fn}$.

\section{Discussion}

As described above, $1 \mathrm{MeV}$ electron irradiation at $300 \mathrm{~K}$ under $\mathrm{N}_{2}$ atmosphere significantly affected the optical properties of the (GACsMAFA) $\mathrm{Pb}(\mathrm{IBr})_{3}$ layers. The fluence dependence of quadruple HOIPs on the aging behavior (absorbance) and response to the illumination treatment was performed after each aging period (PL and TRPL) that showed the intrinsic defects created upon $1 \mathrm{MeV}$ electron irradiation modified the electronic states that are optically active. Defects can have different types of interaction modes that apparently result in modified electronic states. The modes of interaction that appear predominant in the (GACsMAFA) $\mathrm{Pb}(\mathrm{IBr})_{3}$ HOIP layers are discussed below to elucidate the beneficial effects of electronic irradiation. 


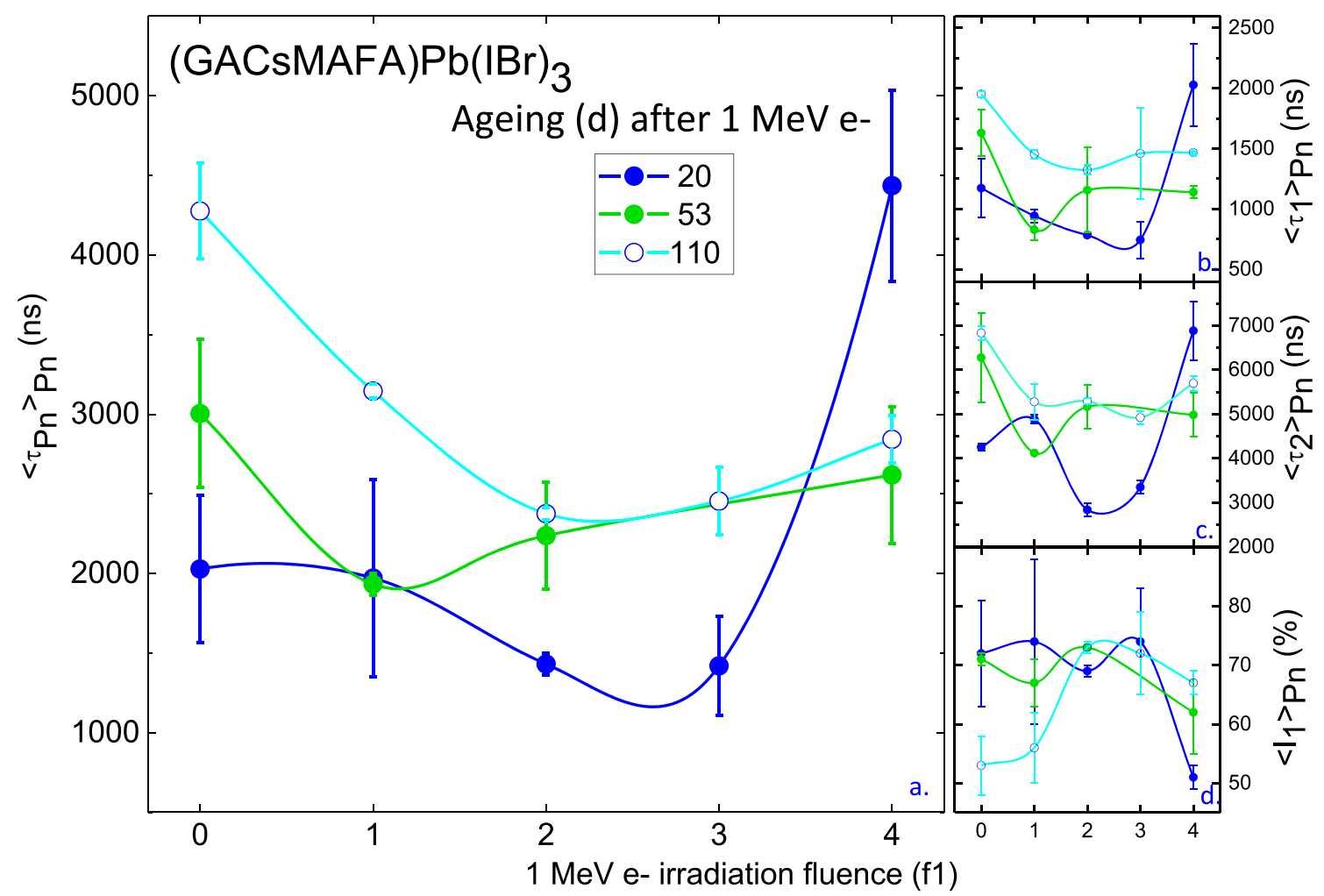

Fig. 7 Fluence dependence of the average lifetime decay spectra recorded after aging and $30 \mathrm{~min}$ of laser illumination (Fig. 5) in (GACsMAFA) $\mathrm{Pb}(\mathrm{IBr})_{3}$ HOIPs for the Ajd-rf-f0 reference layer and the Ajd fn $1 \mathrm{MeV}$-irradiated layers at the fn fluence. The conditions for the

Irradiation versus native defects: effect on A0d optical absorption This paragraph shows that there are shallow and deep A0d localized electronic states in the $7 \mathrm{~d}$ aged A0d rf- $\mathrm{fO}$ and $1 \mathrm{MeV}$ e-irradiated A0d fn layers. The populations of A0d defects that are respectively associated to these distributions are modified by the $1 \mathrm{MeV}$ e-irradiation. The concentration/ nature of the $\mathrm{A} 0 \mathrm{~d}$ defect populations and their contribution to the A0d localized electronic states evolve with the A0d fn- $\mathrm{c}_{\mathrm{ir}}$ concentrations of irradiation defects.

Native defects in the $7 \mathrm{~d}$ aged A0d_rf-f0 reference layer The energy-dependence of the absorbance in the A0d rf-f0 reference layer corresponds to a layer that has aged $7 \mathrm{~d}$ in dark under $\mathrm{N}_{2}$ atmosphere at room temperature $\left(\mathrm{N}_{2}\right.$, dark, $\left.\sim 300 \mathrm{~K}\right)$. This energy-dependence displays the typical shape reported for similar quadruple cation based HOIPs $[4,5]$. For the thickness of the layer, $d=500 \mathrm{~nm}$, the absorption coefficient, $\alpha(\mathrm{E})$, has a value in $\mathrm{cm}^{-1}: \alpha(\mathrm{E})=4.6110^{4} \mathrm{~A}(\mathrm{E})$ (Fig. 2). At $2 \mathrm{eV}$, the value $\sim 8 \times 10^{4} \mathrm{~cm}^{-1}$ is quite consistent with those found for example in $\mathrm{MAPbI}_{3}[13,17,19]$ and of the same order as those in other semiconductors used in photovoltaic applications. This shows that the $7 \mathrm{~d}$ aging has little effect on the absorption coefficient in the higher energy range $\mathrm{E}(\mathrm{eV}) \geq 1.68$.

As determined in Sect. 3, the optical bandgap at room temperature in A0d_rf-f0 has a value A0d_rf-f0_Eg $\mathrm{Eg}_{\sim 300 \mathrm{~K}}$ in $\mathrm{eV}$ of
Ajd aging are $\mathrm{j}(\mathrm{d})=20 / 53 / 110$ under $\mathrm{N}_{2}$ atmosphere in dark at $\sim 300 \mathrm{~K}$ The TRPL spectra are recorded using a nano-LED_482nm, $14 \mathrm{pJ} / \mathrm{pulse}$ energy, $100 \mathrm{ps}$ pulse duration, and $10 \mathrm{kHz}$ repetition rate. The laser spot has a diameter of about $250 \mu \mathrm{m}$ diameter

1.63(1) (Table 2). In A0d rf-f0, the onset for the optical transitions is $\sim 1.56(1) \mathrm{eV}$. The absorption edge has a width where the logarithm of the absorbance increases linearly as the energy increases up to $\sim 1.68(1) \mathrm{eV}$ (Fig. 1). This corresponds to an exponential smearing of the absorption coefficient (Fig. 1), $\alpha(\mathrm{E})=\alpha^{\circ} \cdot \exp \left(\left(\mathrm{E}-\mathrm{E}^{\circ}\right) / \mathrm{Eu}(\mathrm{T}(\mathrm{K}))\right.$ with an Urbach energy $([20]$ and references herein), $\mathrm{E}_{\mathrm{U}}(\mathrm{T}(\mathrm{K}))$, characteristic of the energy width of the absorption edge. The Urbach energy in the A0d rf$\mathrm{fO}$ reference layer has a value in $\mathrm{meV}, \mathrm{A} 0 \mathrm{~d}$ rf- $\mathrm{f0}-\mathrm{Eu}_{\sim 300 \mathrm{~K}}-52(2)$ (Table 4). This value in A0d rf-f0 can be compared to the value determined in a pristine layer prepared in the same way as rf-f0 and rf-17. The energy-dependence of the absorptance determined in this layer via photothermal deflection spectroscopy (PDS) measurements at room temperature in perfluorinated liquid yields a value of 1.64(1) eV for the bandgap and of 18(2) meV for the Urbach energy. The value for the bandgap is quite consistent with that in A0d_rf-f0. The value for the Urbach energy is significantly lower than in A0d_rf-f0.

The 7 days aging $\left(\mathrm{N}_{2}\right.$, dark, $\left.\sim 300 \mathrm{~K}\right)$ appears to have little effect on the bandgap value while it affects the Urbach energy in the $\mathrm{GA}_{0.015} \mathrm{Cs}_{0.046} \mathrm{MA}_{0.152} \mathrm{FA}_{0.787} \mathrm{~Pb}\left(\mathrm{I}_{0.815} \mathrm{Br}_{0.185}\right){ }_{3}$ layers. The bandgap values in these layers are slightly higher, $\geq$ $0.03(1) \mathrm{eV}$, than those reported in layers prepared from precursor solutions with different relative molarities as concern the mixing of the four cations as well as of the halide anions 
$[4,5]$. In [4], GA cations were gradually incorporated in $\mathrm{Cs}_{0.1}\left(\mathrm{FA}_{0.83} \mathrm{MA}_{0.17}\right)_{0.9} \mathrm{~Pb}\left(\mathrm{I}_{0.83} \mathrm{Br}_{0.17}\right)_{3}$ perovskite films deposited on glass/ITO substrates. The GA content in the CsMAFA precursor solutions was varied by adding $\mathrm{GAPbI}_{3}$ with increasing molar ratio from $10 \%$ to $40 \%$. The bandgap energy was found at around $1.61 \mathrm{eV}$ independently on the amount of $\mathrm{GA}$ addition in the precursor solutions. In [5], $\mathrm{Cs}_{0.05}\left(\mathrm{FA}_{0.83}\left(\mathrm{MA}_{1-\mathrm{x}} \mathrm{GA}_{\mathrm{x}}\right)_{0.17}\right)_{0.95} \mathrm{~Pb}\left(\mathrm{I}_{0.83} \mathrm{Br}_{0.17}\right)_{3}$ perovskites films on glass/FTO substrates were characterized as a function of the MA to GA cation stoichiometry. Layers were prepared from different precursor solutions where GA cations gradually replace MA cations. In this case, the optical bandgap energy value increases with increasing GA stoichiometry, from $1.60 \mathrm{eV}$ for $x=0.1$ to $\sim 1.63 \mathrm{eV}$ for $x=0.6$. From DFT calculations, it is generally the $\mathrm{PbX}_{6}$ octahedra that are considered as the main factor determining the bandgap value $[2,21]$. The size of the cations on the $\mathrm{A}$-sites of the $\mathrm{APbX}_{3}$ perovskites can however either contract or expand the crystal lattice, thereby altering the optical bandgap [22]. The small differences in the bandgap values reported by $[4,5]$ with those reported here may rather be ascribed to the slight differences in the halide anion stoichiometry.

The exponential increase of the absorbance at the absorption edge is related to the existence of transitions involving electronic states lying in tails of the density of states in the valence and conduction bands (band tails) ([20] and references herein). The existence of native near-band edge electronic states has already been reported for HOIPs. From external quantum efficiency (EQE) measurements at the absorption edge, an Urbach energy of 16(1) $\mathrm{meV}$ is derived for the active $\mathrm{MAPbI}_{3}$ layers in n-i-p or p-i-n solar cells, independently on the preparation procedure - vapor deposition growth or coating from solutions [23]. The presence of band tails in pristine $\mathrm{FAPbI}_{3}$ was also inferred from the PL temperaturedependence [24]. The authors report the presence of two peaks in the PL spectra obtained at low-temperature PL measurements performed at increasing illumination intensity. The higher energy peak with increasing temperature displays a blue-shift to higher energy. On the contrary, the lower energy peak with increasing temperature exhibits a redshift. The blueshift is the usual behavior observed in HOIPs ([24] and references herein). It was attributed by Wright et al. to the radiative recombination of electron-hole delocalized in the conduction-valence bands and identified as excitons from 10 to $50 \mathrm{~K}$ in $\mathrm{FAPbI}_{3}$. The lower peak was related to trap mediated radiative emission.

The 7 days aged A0d rf-f0 reference layer $\left(7 \mathrm{~d}, \mathrm{~N}_{2}\right.$, dark, $300 \mathrm{~K}$ ) has a much larger Urbach energy than the pristine rf-17 layer, by about a factor $\sim 3.3$. It follows that the tail distribution of shallow localized electronic states is broader in A0d rf-f0 than in rf-17. The question is whether this difference reflects uncontrolled variability in the layer preparation and/or the $7 \mathrm{~d}$ aging. One notices that the bandgap in the pristine layer prepared with the same procedure as $\mathrm{rf}-17$ and A0d_rf-f0, 1.64(1) eV, is in excellent agreement with the 1st PL peak energy in the pristine rf-17 layer, $1.6426(5) \mathrm{eV}$. This strongly supports that the $7 \mathrm{~d}$ aging rather than variability during layer preparation plays a dominant role in the broadening of the band tails. This broadening indicates an increase of the concentration of the defects that give rise to the shallow localized electronic states in the bandgap tail. The question is whether these $7 \mathrm{~d}$ aging-induced defects in the A0d_rf-f0 reference layer have the same nature as those that are generated during the preparation. One possibility is that the interaction between the layer surface and the $\mathrm{N}_{2}$ atmosphere induces structural disorder (vacancies, interstitials, dislocations, site substitutions) or compositional one (deviation from stoichiometry, doping) that are rather specific to the $\left(\mathrm{N}_{2}\right.$, dark, $300 \mathrm{~K}$ ) aging processes than to the preparation.

At higher and lower energy than the absorbance edge, the A0d rf-f0 reference layer absorption coefficient varies slowly in $\mathrm{A} 0 \mathrm{~d}$ _rf-f0. The ratio of its values in the lower range to the higher energy range mainly reflects the height of the absorbance steep step. For example, as energy decreases from 2 to $1.2 \mathrm{eV}$, the absorption coefficient decreases by factor $\alpha_{\sim 300 \mathrm{~K}}(1.2) / \alpha_{\sim 300 \mathrm{~K}}(2) \sim 1.1 \times 10^{-1}$. This factor is much smaller than in the pristine layer prepared in the same condition as $\mathrm{A} 0 \mathrm{~d}$ rf- $\mathrm{f0}$ and $\mathrm{rf}-17$. The ratio between the two values is in this layer, $\sim 8.2 \times 10^{-4}$. Such variations are also observed in other HOIPs. For example, the ratio for $\alpha_{\sim 300 \mathrm{~K}}(1.53) /$ $\alpha_{\sim 300 \mathrm{~K}}(1.7)$ as energy decreases from 1.7 to $1.53 \mathrm{eV}$ are in $\mathrm{MAPbI}_{3}$ about $\sim 10^{-2}[13]$ or $2.5 \times 10^{-3}$ [25], and for example $\sim 1.3 \times 10^{-1}$ in $\mathrm{Cs}_{0.17} \mathrm{FA}_{0.83} \mathrm{~Pb}\left(\mathrm{Br}_{0.2} \mathrm{I}(0.8)\right)_{3}[18]$, respectively.

Below the absorption edge, for $1.2 \leq \mathrm{E}(\mathrm{eV}) \leq 1.6$, the A0d_rf-f0 reference layer absorption coefficient slowly decreases with decreasing energy towards mid-gap and, as seen above, its values remain quite high compared to those in the higher energy range above the absorption edge. This indicates that, beyond the absorption onset towards mid-gap, there is a A0d_rf-f0 distribution of deep localized electronic states. This distribution is present in the 7 days aged A0d_rf-f0 layer and absent in the pristine one. It reflects the differences in the layer quality as concern structural and compositional disorders. Distributions of deep electronic states have also been observed in $\mathrm{MAPbI}_{3}$ layers $[13,23]$. More generally, in semiconductors, defects giving rise to localized electronic states lying deep in the forbidden gap may modify the sub-band optical behavior [26]. One of the known examples is the native defect energy level known as EL2 in GaAs [27]. This localized energy level is located at $0.75 \mathrm{eV}$ below the conduction-band extremum. When the EL2 defects are present, this level can completely dominate the sub-bandgap absorption at $10 \mathrm{~K}$. Another example for amorphous silicon deposited on sapphire (SOS) is the effect on absorption of ion implantation [28]. The authors report the existence of some new features that appear after implantation in the subgap region of absorption at $300 \mathrm{~K}$. 
The authors attribute them to the formation of divacancy defects in ion-implanted amorphous silicon. Another effect is that ion-implantation induced an increase of the absorption coefficient in the sub-band region. This absorption increase is attributed to the formation of a damaged crystalline silicon or a co-existence of amorphous and crystalline damaged silicon phases.

In summary, the $7 \mathrm{~d}$ aging $\left(\mathrm{N}_{2}\right.$, dark, $\left.\sim 300 \mathrm{~K}\right)$ in the $\mathrm{GA}_{0.015} \mathrm{Cs}_{0.046} \mathrm{MA}_{0.152} \mathrm{FA}_{0.787} \mathrm{~Pb}\left(\mathrm{I}_{0.815} \mathrm{Br}_{0.185}\right)_{3} \mathrm{~A} 0 \mathrm{~d}$ rff-f0 reference layer generates "native" $7 \mathrm{~d}$ aging-induced defects that give rise to A0d_rf-f0 deep localized electronic states. Defects that generate A0d_rf-f0 shallow levels are also introduced. On the basis of the absorbance, it is difficult to determine whether the $7 \mathrm{~d}$ aging-induced A0d_rf-f0 shallow defects have the same nature as those present in a pristine layer or are different.

\section{Irradiation versus native defects in the A0d_fn layers aged $7 d$} before irradiation In the irradiated A0d_fn layers, it is assumed that the $7 \mathrm{~d}$ aging before irradiation induces native $7 \mathrm{~d}$ aging-induced defect populations similar to those present in the A0d_rf-f0 reference layer. The absorption coefficient in the higher energy range above absorption edge, $\sim 1.68 \leq$ $\mathrm{E}(\mathrm{eV}) \leq 2.12 \mathrm{eV}$, and the bandgap remain independent on fluence in the A0d_fn layers (Table 2). For both, the values in the irradiated A0d_fn layers are the same as in the A0d_rff0 layer. This is a clear evidence that $1 \mathrm{MeV}$ e-irradiation at fluences, $\mathrm{fl} \leq \mathrm{fn} \leq \mathrm{f} 4$, introduces irradiation defect populations with A0d_fn- $c_{i r}$ concentrations low enough to preserve the layer composition stoichiometry and crystalline structure that exist in the A0d_rf-f0 layer.

There is an exponential smearing of the absorption edge in the irradiated A0d_fn layers that yields to fluence dependent Urbach energies (Table 4). The values are in meV, A0d_fn$\mathrm{E}_{\mathrm{u} \sim 300 \mathrm{~K}}$ :_f1-f2-f3-47(2); f4-50(2). When compared to the value in A0d_rf-f0 reference layer, $52(2) \mathrm{meV}$, the width of the A0d_fn band tails goes through a weak minimum as the concentration of irradiation defects increase in the range $\mathrm{fl}<\mathrm{fn} \leq$ f3. This minimum suggests the A0d_fn tails of shallow localized electronic states evolve with the irradiation defects according to different modes depending on their $\mathrm{A} 0 \mathrm{~d}$ _fn- $\mathrm{c}_{\mathrm{ir}}$ concentration. The A0d_fl-c $\mathrm{c}_{\mathrm{ir}}$ concentration of irradiation defects appears to be sufficient to slightly reduce the width of the initial band tails. The irradiation defects seem to partially remove the initial native ones. As fluence increases further, it is only the higher A0d f4- $\mathrm{c}_{\mathrm{ir}}$ concentration of irradiation defects that appears to be sufficient for contributing also to the band tails and to broaden their distribution.

In the lower energy range below the absorption onset, $1.2 \leq \mathrm{E}(\mathrm{eV}) \leq 1.6 \mathrm{eV}$, the absorption coefficients in the irradiated $\mathrm{A} 0 \mathrm{~d}$ fn layers have a slow decrease towards mid-gap and have still high values. For example, the value at $1.2 \mathrm{eV}$ is only one order of magnitude less than at $2 \mathrm{eV}$. This shows the existence of electronic states in the sub-bandgap region which extends towards mid-gap. The baseline of the absorption coefficient reaches a minimum with increasing fluence for $\mathrm{f}$. This indicates that the initial sub-bandgap distribution of electronic states below $1.6 \mathrm{eV}$ present in the reference $\mathrm{A} 0 \mathrm{~d}$ rf-f0 layer is affected by the introduction of irradiation defects. The A0d fn irradiated layers are more transparent than the reference $\mathrm{A} 0 \mathrm{~d} \mathrm{rf}-\mathrm{f} 0$ one. This suggests that irradiation reduces the density of the "native" $7 \mathrm{~d}$ aging-induced deep electronic states or affects the absorption oscillator strength by changing their nature. In the first case, the role of the irradiation defects in A0d_fn implies a destruction of the initial deep electronic states observed in A0d_rf-f0. In the second case, it implies a progressive replacement of those with irradiation-induced deep electronic states. The competition between these two processes can explain why the absorbance in the lower energy region goes through a minimum for the $\mathrm{f} 3$ fluence. As fluence increases up to $\mathrm{f} 2$, the predominant role of the irradiation defects is to progressively remove the contribution of the native deep electronic states to the optical absorption. At $\mathrm{f} 3$, the concentration of irradiation defects, A0d_f3- $\mathrm{c}_{\mathrm{ir}}$, reaches a threshold value sufficient for partially removing the initial deep electronic states defects in A0d_rf-f0. The absorbance for $\mathrm{f} 3$ then goes through a minimum. Above the $\mathrm{f} 3$ fluence, as the concentration further increases to A0d_f4- $\mathrm{c}_{\mathrm{ir}}$, their role as producing deep electronic states within the bandgap becomes predominant. It is reflected by the absorbance increase as fluence increases from $\mathrm{f} 3$ to $\mathrm{f} 4$.

The absorbance oscillations in the energy region $1.2 \leq \mathrm{E} \leq$ $1.6 \mathrm{eV}$ have amplitudes that are higher in the irradiated Ajd_fn layers than in the reference A0d_rf-f0 one. In the case of thin layers/films on thick substrates, oscillations may appear in the transmission and reflection spectra that arise from interference fringes produced at the interface between the layer/film and its substrate [29]. The stronger amplitude of the oscillations in the A0d_fn layers suggests that roughness is stronger in A0d_fn than in A0d_rf-f0 [30].

In summary, for the $1 \mathrm{MeV}$ electron irradiation conditions chosen in this work, the fluence effects observed for the A0d absorbance in the (GACsMAFAPb)(IBr) ${ }_{3}$ layers set-18 are fully consistent with the existence of distributions of shallow and deep sub-bandgap electronic states. The density and/or the nature of the localized states change with the concentration of irradiation defects in the A0d_fn layers. At the low A0d_fn- $\mathrm{c}_{\text {ir }}$ concentrations in the fluence range $\mathrm{fl} \leq \mathrm{fn} \leq \mathrm{f} 3$, the irradiation defects most likely decrease the density of the shallow and deep electronic states initially present. Their main role is then to partially remove the native $7 \mathrm{~d}$ aging-induced defects that give rise to the initial distributions, i.e., the defects that exist after the $\left(7 \mathrm{~d}, \mathrm{~N}_{2}\right.$, dark, $\left.\sim 300 \mathrm{~K}\right)$ initial aging. At the higher A0d $\mathrm{f}-\mathrm{c}_{\mathrm{ir}}$ concentration, for the f4 fluence, their main role is to introduce shallow and deep localized electronic sates that differ from the initial ones. 


\section{Electron irradiation induced-aging of optical absorption} Absorbance gives access to four types of variations that can be examined to determine how the initial $7 \mathrm{~d}$ aging-induced defects evolve with aging duration in the Ajd rf-f0 reference layer and interact with the irradiation defects in the $1 \mathrm{MeV}$ eirradiated Ajd_fn layers $(j(d)=53,110)$. These variations are those of (i) the bandgap, (ii) the Urbach energy, (iii) the energy dependence of the absorption coefficient in the higher energy range above the absorption edge, $\mathrm{E}(\mathrm{eV}) \geq 1.68$, and (iv) the energy dependence of the absorption coefficient in the lower energy range below the absorption edge, $\mathrm{E}(\mathrm{eV}) \leq 1.6$.

Aging-induced defects in the A53d/A110d_rf-f0 layer The main effect of increasing the aging duration in the A53d/A110d_rf-f0 layer is to induce variations of the (ii) and (iii) types. (ii) The increase of aging duration produces a distribution of shallow electronic states that slightly broadens as aging duration after preparation increases from $7 \mathrm{~d}(\mathrm{~A} 0 \mathrm{~d})$ to $60 \mathrm{~d}(\mathrm{~A} 53 \mathrm{~d})$, and further, till $117 \mathrm{~d}(\mathrm{~A} 110 \mathrm{~d})$. (iii) In the higher energy range above the absorption edge, $\mathrm{E}(\mathrm{eV}) \geq 1.68$, the aging between $7 \mathrm{~d}(\mathrm{~A} 0 \mathrm{~d})$ to $60 \mathrm{~d}(\mathrm{~A} 53 \mathrm{~d})$ induces a decrease of the absorption coefficient that is much stronger above $\mathrm{E}(\mathrm{eV}) \geq 2.2$ than below. The further aging from $60 \mathrm{~d}(\mathrm{~A} 53 \mathrm{~d})$ to $117 \mathrm{~d}(\mathrm{~A} 110 \mathrm{~d})$ has a much smaller effect. It only slightly decreases the absorption coefficient. It follows that the increase of aging duration after preparation to $60 \mathrm{~d}(\mathrm{~A} 53 \mathrm{~d})$ and further, till, $117 \mathrm{~d}(\mathrm{~A} 110 \mathrm{~d})$ introduces two kinds of Ajd_rf-f0 aging-induced defects population. The first kind rather affects the shallow localized electronic states. The second one rather affects the absorption coefficient in the higher energy range above the absorption onset.

Irradiation induced-aging defects in the A53d/A110d_fn layers The concentration of irradiation defects, A0d fn- $\mathrm{c}_{\mathrm{ir}}$, introduced in the A0d_fn layers induces variation of the four types as aging duration after irradiation increases up to $53 \mathrm{~d}(\mathrm{~A} 53 \mathrm{~d})$ and $110 \mathrm{~d}(\mathrm{~A} 110 \mathrm{~d})$. (i)The optical bandgap in the A53d/110d_fn irradiated layers depends on the fn fluence. Compared to the respective values in A53/A110d_rf-f0 reference layer, it has a systematic tendency to slightly shrink as the irradiation defect concentration reaches a threshold value corresponding to the $\mathrm{f} 2$ fluence. The irradiation-induced bandgap narrowing for $\mathrm{f} 2 \leq \mathrm{fn} \leq \mathrm{f} 4$ depends on the aging duration and has values in $\mathrm{eV}$, Ajd fn- $\mathrm{dEg}_{\sim 300 \mathrm{~K}}$ : A53d f2-f3-f4-($0.02(2))$; A110d_f2_(- $0.02(2))$, A110d_f3-f4_(-0.03(2)). (ii) The absorbance exhibits an energy exponential dependence in the steep part at the absorption edge of the A53/ A110d fn layers. The associated Ajd fn Urbach energy in the $\mathrm{A} 53 \mathrm{~d} / \mathrm{A} 110 \mathrm{~d}$ fn layers goes through a maximum as the A0d_fn-c $c_{i r}$ irradiation defect concentration increases with increasing fluence. The maximum for both aging durations is reached for the $\mathrm{A} 0 \mathrm{~d}$ f $2-\mathrm{c}_{\mathrm{ir}}$ defects concentration produced at the $f 2$ fluence (Table 4 ). The maximum value of the Ajd f2
Urbach energy at $\mathrm{f} 2$ increases with aging duration and has values in meV, Ajd_f2-Eu $\mathrm{Eu}_{300 \mathrm{~K}, \max }$ : A53d_f2-83(2), A110d f2-100(2). (iii) In the higher energy range above the absorption edge $(\mathrm{E}(\mathrm{eV}) \geq 1.68)$, the absorption coefficient decreases with increasing $\mathrm{A} 0 \mathrm{~d}$ fn- $\mathrm{c}_{\mathrm{ir}}$ irradiation defect concentration. The decrease is stronger above $\mathrm{E}(\mathrm{eV}) \geq 2.2$ than below. Furthermore, it reaches a saturation level at each $\mathrm{A} 53 \mathrm{~d} /$ $110 \mathrm{~d}$ aging as the A0d fn irradiation defects reaches a concentration threshold, A0d_f2-c $\mathrm{c}_{\mathrm{ir}}$, corresponding at the f2 fluence. (iv) In the lower energy range below the absorption edge, $1.2 \leq \mathrm{E}(\mathrm{eV}) \leq 1.6$, the $\mathrm{A} 53 \mathrm{~d} / \mathrm{A} 110 \mathrm{~d}$ absorbance depends on the A0d fn- $\mathrm{c}_{\mathrm{ir}}$ concentrations of irradiation defects. As aging duration increases, 47d(A110d-A53d), the Ajd_fn layers absorption increases in this energy region. At each A53d/A110d aging, the most absorbing Ajd fn layers correspond to the A0d_f1- $\mathrm{c}_{\text {ir }}$ and A0d_f2- $\mathrm{c}_{\mathrm{ir}}$ irradiation defect concentrations introduced at the $\mathrm{f} 1$ and $\mathrm{f} 2$ fluences, respectively.

Irradiation induced-A53d/A110d aging processes When the effects of increasing aging duration are compared for the absorbance in the irradiated Ajd_fn and the reference Ajd_rf-f0 layers, there is clear evidence that the A0d_fn irradiation defects affect the $53 \mathrm{~d} / 110 \mathrm{~d}$ aging processes. The A0d_f $2-\mathrm{c}_{\mathrm{ir}}$ concentration of irradiation defects introduced at the $f 2$ fluence in the A0d_f2 layer appears as a threshold. At this threshold concentration, the aging processes in the $\mathrm{f} 2$ layer (i) start to shrink the A53d/A110d_fn bandgaps and (ii) produce A53d/A110d_fn band tails with a maximal width. The width is about $36-40 \%$ larger in the A53d/A110d_f2 layer than in the A53d/A110d_rffo reference one. At this threshold concentration, the aging processes (iii) cease to affect the A53d/A110d_fn absorption coefficients in the higher energy range above the absorption edge. At this threshold concentration, the aging processes (iv) induce the higher A53d/A110d_fn subgap absorbance. These correlated effects lead to conclude that the irradiation-aging induced defect populations differ below and above the A0d_f2-c $c_{\text {ir }}$ irradiation defect concentration. Depending on the balance between the competitive chemical reactions occurring during aging, the concentration and/or the nature of the shallow and deep localized electronic states change below and above this threshold concentration.

In summary, the energy-dependence of absorbance in the Ajd_rf-f0 reference layer has a (jd, $\mathrm{N}_{2}$, dark, $300 \mathrm{~K}$ ) aginginduced evolution that leads to identify three different kinds of Ajd_rf-f0 defect populations. One kind (i) induces a slight Ajd_rf-f0 bandgap shrinkage and, consequently, corresponds to a high aging-defect concentration, Ajd $\mathrm{rf}-\mathrm{fO} \mathrm{cl}_{\mathrm{ag}}$. The two others (ii) and (iii) generate shallow and deep Ajd rf-f0 electronic states, respectively. The concentration associated to the shallow ones is so high that the states overlap and give rise to a quasi-continuous distribution of energy levels in the Ajd rf-f0 band tails.

The energy-dependence of absorbance in the Ajd fn irradiated layers has a $\left(\mathrm{N}_{2}\right.$, dark, $\left.\sim 300 \mathrm{~K}\right)$ aging-induced 
evolution consistent with the existence of three types of Ajd_fn defect populations that generates (i) Ajd_fn bandgap shrinkage, (ii) shallow and (iii) deep Ajd_fn electronic states, respectively. None of the three irradiation-aging induced populations displays a monotonous evolution as a function of the irradiation defects concentrations (A0d_fn- $\mathrm{c}_{\text {ir }}$ ) with increasing aging. The three types of (i)-(iii) irradiation-induced aging populations in the Ajd_fn irradiated layers differ by their concentration and/or nature from the aging-induced ones in the Ajd_rf-f0 reference layer. The increase of aging duration tends to systematically increase the contribution of the Ajd_fn deep electronic states to the Ajd_fn sub-bandgap absorbance.

Natural and irradiation-induced-aging defects: PL after 1$3 \mathrm{~min}$ of illumination (454 nm, $1.1 \mathrm{~W} / \mathrm{cm}^{2}$, air, $300 \mathrm{~K}$ ) When illumination starts in the aged $\operatorname{Ajd}(j(d)=20,53,110)$ rf-f0 reference and $1 \mathrm{MeV}$ e-irradiated fn layers, as discussed above, three types of irradiation aging-induced Ajd defects are initially present. The first one induces the slight bandgap Ajd_fn shrinkage that depends on aging and fluence. The second one generates the Ajd band tails of shallow electronic states. The third one gives rise to the Ajd sub-bandgap distributions of deep electronic states. For the $20 \mathrm{~d}$ aged states of the layers, the properties of these distributions are inferred from those in the A0d and A53d aged states. As a result of the "native" Ajd aging-induced distributions, the Fermi level position in dark can vary in the Ajd_rf-f0 and Ajd_fn layers. Under illumination, the electron and hole quasi-Fermi level respective positions may also depend on the layer states ([31] and references herein). Furthermore, the elimination of the metastable A0d_fn irradiations defects in the Ajd_fn layers can have properties that differ under illumination from those in dark. The reason is that the defect migration energy and the rates of the chemical reactions involving the defects are expected to depend on the occupancy of the defect charge states. Those occupancies are controlled by the respective positions of the defect ionization levels and of the Fermi level in dark or those of the electron and hole quasi Fermi levels under illumination.

For the A20d aging, the values for the A20d_fn bandgap shrinkage are expected between those for the A0d_fn and A53d_fn ones (Table 2). For the shallow electronic states, the Urbach energies associated to the A20d_fn near bandgap distributions are inferred from the values determined for the A0d and A53d ones (Table 4). For the A20d_rf-f0 reference layer, the value is most likely equal to their average, 53(2) $\mathrm{meV}$ (Table 4). For the irradiated A20d fn layers, it seems reasonable to assume that the A20d fn Urbach energies are for each fn fluence between the A0d fn and A53d fn values (Table 4), respectively. The indetermination of the value covers an order of magnitude as the concentration of irradiation defects increases with increasing fluence from f1 to $\mathrm{f} 4$. This indetermination is minimal for the fluence $\mathrm{fl}$, $3(50-47) \mathrm{meV}$. It goes through a marked maximum for the fluence $\mathrm{f} 2,36(83-47) \mathrm{meV}$. Then, as the irradiation defect concentrations increases further at higher fluence, the range of indetermination becomes quasi-constant and, about, twice less than at $\mathrm{f} 2$ with values for f3-14(6147) $\mathrm{meV}$ and f4-17(67-50) $\mathrm{meV}$.

For the deep electronic states, their A20d sub-bandgap distribution is expected to affect the energy dependence of the absorption coefficient in the A20d_rf-f0 reference and A20d_fn irradiated layers and induce variations between those due to the A0d and A53d sub-bandgap distributions. For all the fluences, except $\mathrm{f} 2$, these distributions are expected to be less absorbent in the A20d_fn layers than in the A20d_rf-f0 one. There is an indetermination for the $\mathrm{f} 2$ fluence. The question is whether the sub-bandgap distribution during the $20 \mathrm{~d}$ aging remains less or becomes more absorbent in A20d_f2 than in A20d_rf-f0. Indeed, from less absorbent in the A0d_f2 state than in A0d_rf-f0, the distribution becomes more absorbent in the A53d_f 2 one than in A53d_rf-f0.

First PL spectra in the pristine rf-17 and aged Ajd_rf-fo layers For the pristine rf-17 layer, the 1st PL spectrum recorded after $1 \mathrm{~min}$ of $488 \mathrm{~nm}$ illumination in air can be considered as defining the quality of the pristine state of a (GACsMAFA)Pb(IBr) $)_{3}$ layer. For the layer set-18, the 1st PL spectra are recorded after $3 \mathrm{~min}$ of $454 \mathrm{~nm}$ illumination in air. These spectra in Ajd_rf-f0 define the quality of a layer where processes occurring during $\left(\mathrm{N}_{2}\right.$, dark, $\left.\sim 300 \mathrm{~K}\right)$ aging after preparation are coupled to those occurring during $3 \mathrm{~min}$ illumination at $454 \mathrm{~nm}$ in air performed after each aging period. One question is whether the radiative recombination in the pristine rf-17 and aged Ajd_rf-f0 reference layers involves only band-to-band carrier recombination or involves also radiative recombination via carrier traps.

The difference between the values of the bandgap and 1st PL peak energy depends on the layer state (Table 5). For the pristine state, there is an excellent agreement between those two values, $1.64(1) \mathrm{eV}$ in the pristine layer -prepared as rf-17and the 1st PL peak recorded in rf-17 after 1 min of illumination, $1.6426(5) \mathrm{eV}$. This suggests that it is rather band-to-band radiative recombination that takes place in the pristine $\mathrm{GA}_{0.015} \mathrm{Cs}_{0.046} \mathrm{MA}_{0.152} \mathrm{FA}_{0.787} \mathrm{~Pb}\left(\mathrm{I}_{0.815} \mathrm{Br}_{0.185}\right)_{3}$ rf-17 layer. In the aged Ajd_rf-f0 reference layer, the energy differences, Ajd_rf-f0- $[\mathrm{Eg}-\overline{\mathrm{E}} \mathrm{p}(1 \mathrm{st})]$, remain quasi-constant $\sim 0.04(1) \mathrm{eV}$ as the aging duration increases (Table 5). This is independent of the tendency (at the limit of detection) for the bandgap to shrink by $-0.02(2) \mathrm{eV}$ in Ajd_rf-f0 during the 110d aging from A0d to A110d. The differences are slightly smaller, by $\sim 10-20 \mathrm{meV}$, than the initial Urbach energy for the Ajd rf-f0: $\mathrm{A} 0 \mathrm{~d} / 53 \mathrm{~d} / 100 \mathrm{~d}$ rff-f0-Eu $\mathrm{E}_{\sim 300 \mathrm{~K}}-52 / 53 / 59$ (2). In addition, there is no correlation with increasing aging between the variations of the 1st PL peak energy and FHWM in the reference Ajd_rf- 
Table 5 Difference between the first photoluminescence peak and bandgap energy after aging in (GACsMAFA) $\mathrm{Pb}(\mathrm{IBr})_{3}$ HOIPs for the reference Ajd-rf-f0 and the $1 \mathrm{MeV}$ e-irradiated Ajd_fn layers at the fn fluence. The conditions for the Ajd aging are $\mathrm{j}(\mathrm{d})=0 / 20 / 53 / 110$ under $\mathrm{N}_{2}$ atmosphere in dark at $\sim 300 \mathrm{~K}$

\begin{tabular}{|c|c|c|c|c|c|c|c|c|c|}
\hline$\left(\operatorname{Ep}\left(\mathrm{Ajd} \_f n\right)-E g\left(A j d \_f n\right)\right)(e V)$ & rf-f0 & $\mathrm{fl}$ & $\mathrm{f} 2$ & $\mathrm{f} 3$ & $\mathrm{f} 4$ & vsf0_fl & vsf0_f2 & vsf0_f3 & vsf0_f4 \\
\hline $\operatorname{Ep}(\mathrm{A} 20 \mathrm{~d})-\operatorname{Eg}(\mathrm{A} 0 \mathrm{~d})$ & $-0.04(1)$ & $-0.04(1)$ & $-0.04(1)$ & $-0.04(1)$ & $-0.06(1)$ & $0.00(2)$ & $0.00(2)$ & $0.00(2)$ & $-0.02(2)$ \\
\hline $\operatorname{Ep}(\mathrm{A} 53 \mathrm{~d})-\mathrm{Eg}(\mathrm{A} 53 \mathrm{~d})$ & $-0.04(1)$ & $-0.04(1)$ & $-0.02(1)$ & - & $-0.03(1)$ & $0.00(2)$ & $0.02(2)$ & - & $0.01(2)$ \\
\hline $\mathrm{Ep}(\mathrm{A} 110 \mathrm{~d})-\mathrm{Eg}(\mathrm{A} 110 \mathrm{~d})$ & $-0.04(1)$ & $-0.03(1)$ & $-0.03(1)$ & $-0.01(1)$ & $-0.01(1)$ & $0.01(2)$ & $0.01(2)$ & $0.03(2)$ & $0.03(2)$ \\
\hline vsA0d_A53d & $0.00(2)$ & $0.00(2)$ & $0.02(2)$ & - & $0.03(2)$ & & & & \\
\hline vsA0d_A110d & $0.00(2)$ & $0.01(2)$ & $0.01(2)$ & $0.03(2)$ & $0.05(2)$ & & & & \\
\hline vsA53d_A110d & $0.00(2)$ & $0.01(2)$ & $0.01(2)$ & - & $0.02(2)$ & & & & \\
\hline
\end{tabular}

f0 layer. The Ajd rf-f0 1stPL peak FWHM goes through a maximum after the A53d aging while the Ajd rf-f0 1st PL peak energy goes on decreasing. This reflects that the Ajd_rf-f0 1st PL spectra are composed of overlapping shallow emission transitions that evolve towards lower energies with increasing aging in the Ajd rf- $\mathrm{fO}$ reference layer. Given the Ajd_rf-f0 1st PL characteristics, the conclusion is that radiative carrier recombination in Ajd_rf-f0 involves shallow energy levels after each Ajd aging.

For most inorganic semiconductors, the broadening of a PL peak may depend on several factors, notably scattering with defects (imperfections, disorder...) as well as electron-phonon coupling $[32,33]$. In pristine HOIPs $-\mathrm{MAPbI}_{3}, \mathrm{MAPbBr}_{3}$, $\mathrm{FAPbI}_{3}, \mathrm{FAPbBr}_{3}$ - layers deposited on quartz substrates, FWHM was extracted from temperature-dependent steadystate PL under vacuum and compared with the emission broadening estimated from DFT and many-body perturbation theory calculations [32]. This broadening was found to mainly arise from the sum of the impurity scattering term and electron-optical phonon scattering. The first term is temperature independent. The second one increases linearly with increasing temperature. At room temperature, the extracted FWHM values in vacuum vary between 0.09 and $0.11 \mathrm{eV}$ and are shown to be in good agreement with the DFT calculations. The values reported in [32] are consistent with those found for the first PL spectra in the pristine rf-17 layer, $0.099(1) \mathrm{eV}$, and in the aged reference Ajd_rf-f0 layer spectra, between $0.110(4)$ and $0.113(4) \mathrm{eV}$ in (air, $300 \mathrm{~K}$ ).

The shallow trap-assisted carrier radiative recombination in the aged reference Ajd_rf-f0 layer has the interesting property that the Ajd_rf-f0 1st PL peak intensity goes through a minimum at A53d. The shallow electronic levels under illumination have an ability to participate to radiative recombination that slightly decreases $(-10 \%)$, during the first $33 \mathrm{~d}(\mathrm{~A} 20 \mathrm{~d}-$ A53d) aging period. This ability is so much restored during the second $47 \mathrm{~d}$ (A53d-A110d) one that the 1st PL peak intensity becomes $80 \%$ higher in A110d_rf-f0 than in A20d_rf-f0. This evolution is singular when compared to the broadening of the initial Ajd near bang gap tails, $1 \mathrm{meV}$ from A20d to
A53d and $6 \mathrm{meV}$ from A53d to A110d. The assumption that the initial Ajd_rf-f0 aging-induced shallow electronic levels directly play a role implies that the $6 \mathrm{meV}$ broadening increases the probability of radiative recombination under 3 min of illumination by $90 \%$ from A53d_rf-f0 to A110d_rff0. It also implies that the $6 \mathrm{meV}$ broadening results in the associated decrease of the 1st PL peak energy of approx. $0.010 \mathrm{eV}$. It seems rather difficult to attribute such effects to the $6 \mathrm{meV}$ broadening. Consequently, this questions the direct role of the initial Ajd_rf-f0 aging-induced shallow electronic levels on the radiative recombination in the Ajd_rf-f0 layer. The initial Ajd_rf-f0 aging-induced deep sub-bandgap electronic levels are potential non-radiative recombination centers that, depending on the occupancy of their ionization levels under illumination, can affect the Ajd rf-f0 1st PL intensities. On the basis of the quasi-absence of variation of the subbandgap absorption during the 47d(A53d-A110d) second aging period, they appear to remain rather stable during this second period. For them to be at the origin of the huge difference (90\%) between the A53d and A110d_rf-f0 1st PL intensities requires that their occupancy is totally different under illumination performed in the aged reference Ajd_rf-f0 layer after the A53d/A110d aging. Such a property seems to be unlikely and, consequently, questions their direct role.

In summary, the features of the evolution of the Ajd_rf-f0 1 st PL peak characteristics with increasing aging in the Ajd_rf-f0 reference layer lead to the conclusion that most likely the first 3 min of illumination already affects both Ajd_rf-f0 distributions of shallow and deep electronic levels. One notices that this is also the case in the pristine layers (Fig. 4) when one compares the initial Urbach energy (18 $\mathrm{meV}$ ) and initial absence of deep subgap states with the PL evolution in $\mathrm{rf}-17$. Between the 3rd and 1st PL spectra in $\mathrm{rf}-17$, the $3 \mathrm{~min}$ instead of $1 \mathrm{~min}$ of illumination already induces a decrease of approx. $-0.026 \mathrm{meV}$ and approx. $-30 \%$ for the energy and intensity, respectively. The nature and density of those illumination-induced defect populations may differ in the Ajd_rf-f0 aged reference layer and the rf-17 pristine one, depending on the chemical reactions that are initiated and 
promoted under $454 \mathrm{~nm} \sim 300 \mathrm{~K}$ illumination in air by the initially different defect populations.

First PL spectra in the irradiated and, subsequently, aged Ajd$\mathrm{fn}$ layers The aging dependence of the 1stPL spectra in the irradiated Ajd fn aged layers is clearly affected by the A0d_fn- $c_{\text {ir }}$ irradiation defect concentrations. After $3 \mathrm{~min}$ of illumination, the Ajd fn-1st PL spectra have emission characteristics (energy, FHWM, intensity) that are controlled by the under-illumination occupancy of the electronic levels. In the Ajd_fn layers, there exist different types of initial electronic levels: (i) the delocalized ones in the conduction/valence bands and, in addition, depending on the A0d fn- $\mathrm{c}_{\text {ir }}$ irradiation defect concentrations, those (ii) localized at irradiationaging defects and those (iii) partially or fully delocalized in irradiation-aging defect bands.

One question is whether the introduction of the $\mathrm{A} 0 \mathrm{~d}$ - fn- $\mathrm{c}_{\mathrm{ir}}$ of the irradiation defects promotes radiative recombination via band-to-band or carrier traps in the Ajd_fn aged irradiated layers. One can examine how the difference between the 1st PL peak energy and the bandgap values, Ajd_fn-[Eg-Ep(1st)] $(\mathrm{eV})$, evolves with the A0d_fn- $\mathrm{c}_{\mathrm{ir}}$ concentration of irradiation defects to determine which radiative process is the most probable. Table 5 shows that the emission energy is lower than the bandgap for every aging duration and fluence. The energy emission is the less different from the bandgap, approx. $0.01 \mathrm{eV}$, for the A110d aging and the higher A0d_fn- $\mathrm{c}_{\mathrm{ir}}$ concentrations of irradiation defects at the f3-f4 fluences. In this case, given this low value, the possibility that the emission corresponds to a band-to-band radiative recombination cannot be totally excluded. Otherwise, the large shifts for the other aging periods and irradiation defect concentrations, approx. 0.06 to approx. $-0.02 \mathrm{eV}$ (Table 5), suggest that the radiative carrier recombination remains assisted by shallow carrier traps.

The detailed examination of the Ajd_fn-1st PL spectra shows that the A0d_fn- $c_{\text {ir }}$ irradiation defect concentrations induces no simultaneous monotonous variations of their characteristics - peak energy, FWHM and intensity. There are however some key trends (i)-(vi) worth noting between their evolution with increasing Ajd aging:

(i) The Ajd_fl-PL(1st) spectra for the lower A0d_fl-cir concentration have energy and FWHM values that are always the higher ones.

(ii) There is an absence of correlation between the Ajd_fnEp(1st) peak energy and initial Ajd_fn Urbach energy variations with increasing Ajd aging.

(iii) The irradiation-induced dispersion of the Ajd fn-1stPL peak energy and FWHM goes through a minimum after the A53d aging.

(iv) For A20d, the A20d fn-Ip(1st) intensity decreases with increasing concentration of irradiation defects:
A 20 d_f $1-I p(1 \mathrm{st})-\sim(-20 \%)>\sim$ A $20 \mathrm{~d}$ f $2-$ Ip $(1 \mathrm{st})>\bar{A} 20 \mathrm{~d} \_\mathrm{f} 3-\mathrm{Ip}(1 \mathrm{st})>>\mathrm{A} 20 \mathrm{~d} \_\mathrm{f} 4-\mathrm{Ip}(1 \mathrm{st})-\sim(-60 \%)$.

(v) The irradiation-induced dispersion of the Ajd_fn-Ip(1st) intensities goes on decreasing with increasing aging.

(vi) Except for the Ajd_f1 layer, the Ajd_fn-1st PL intensity increases with increasing A20d to A110d aging. This property becomes also true in the Ajd_fl layer with increasing A53d to A110d aging.

The trend (i) indicates that the main A20d fn emission transitions involve ionization levels that are the shallower ones at the lower irradiation defects concentration, A0d_f1$\mathrm{c}_{\mathrm{ir}}$. The trend (ii-iii) suggest that the emission transitions due to shallow levels are not directly imposed by change in the occupancy of the electronic levels arising from the initial irradiation-aging near band edge distributions. It rather seems to indicate that illumination generates specific distributions of shallow levels. The trend (iv) shows that the concentrations of A20d_fn non-radiative recombination centers active under illumination increase with the A0d_fn- $\mathrm{c}_{\text {ir }}$ concentrations. The trend (v) shows that the effects of the A0d_fn-cir irradiation defect concentrations on the Ajd_fn non-radiative recombination centers active under illumination decrease with increasing Ajd aging duration. The trend (vi) appears to occur when the aging-induced Ajd_fn bandgap shrinkage yields in the Ajd_fn layers to bandgap values in $\mathrm{eV}, \mathrm{Ajd} \_\mathrm{fn}(\mathrm{Eg}) \leq 1.61(1)$.

In summary, when the $3 \min (454 \mathrm{~nm}$, air, $\sim 300 \mathrm{~K})$ illumination effects are compared in the Ajd_fn irradiated layers and the Ajd_rf-f0 reference one, the most obvious differences concern the Ajd_fn-PL(1st) intensities. The ratios Ajd_fn$\mathrm{Ip}(1 \mathrm{st}) / \mathrm{A} 20 \mathrm{~d}$ rf-f0-Ip(1st) and the ratios Ajd_fn-Ip(1st)/ Ajd_rf-f0-Ip(1st) at each aging are systematically lower than 1. This is clear evidence that the combination of irradiationinduced aging $\left(\mathrm{N}_{2}\right.$, dark, $\left.\sim 300 \mathrm{~K}\right)$ and $3 \mathrm{~min}$ of $454 \mathrm{~nm}$ illumination systematically yields to concentrations of nonradiative recombination centers always higher under illumination in the Ajd_fn irradiated layers than in the Ajd_rf-f0 reference one. There is a decrease of these centers as aging increases from A20d to A110d in the Ajd_fn layers. This aging property is also valid in the aged reference Ajd_rf-f0 layer although associated to a much stronger A110d reduction of the non-radiative recombination centers under illumination. The aging-induced recovery of the $1^{\text {st }} \mathrm{PL}$ intensity is severely damped by the introduction of the A0d_fn- $\mathrm{c}_{\text {ir }}$ irradiation defects. It is worth noting that this irradiation-induced quenching of the aging recovery of the Ajd fn $1^{\text {st }}$ PL intensity occurs without any correlation to the energy of the Ajd fn emission transitions and FWHM associated to shallow levels in the Ajd fn aged irradiated layers. It follows that the radiative shallow and non-radiative, a priori deep, electronic levels under illumination are associated to two different natures of under illumination-active defect population. Most likely, as in the Ajd_rf-f0 reference and pristine rf-17 layer, chemical 
reactions during the ( $3 \mathrm{~min}, 454 \mathrm{~nm}$, air, $300 \mathrm{~K}$ ) illumination modify the initial Ajd fn aging-induced defect population. The kinetics and/or nature of these chemical reactions evolve with the A0d fn- $c_{\text {ir }}$ irradiation defects.

Irradiation-induced aging and illumination defects under continuous illumination: PL versus illumination-time The effect of continuous illumination on the photoluminescence has d i s t i n c t i e f e a t u r e i n t h e $\mathrm{GA}_{0.015} \mathrm{Cs}_{0.046} \mathrm{MA}_{0.152} \mathrm{FA}_{0.787} \mathrm{~Pb}\left(\mathrm{I}_{0.815} \mathrm{Br}_{0.185}\right)_{3}$ layers depending on their quality: pristine (rf-17), aged after preparation (Ajd_rf-f0) or irradiated after $7 \mathrm{~d}$ aging and, further, subsequently aged (Ajd_fn).

Continuous illumination in the pristine rf-17 layer $(488 \mathrm{~nm}$, $2548 \mathrm{~W} / \mathrm{cm}^{2}$, air, $300 \mathrm{~K}$ ). The PL peak energy and FHWM evolutions during the $5 \mathrm{~h}$ of illumination in $\mathrm{rf}-17$ show that the PL spectra are formed of an evolving superposition of components with the contribution of the lower energy components increasing with illumination time. At the end of the $5 \mathrm{~h}$ illumination, the PL peak energy in the pristine rf-17 layer when compared to the bandgap, 1.64(1) eV, display a strong redshift with value in $\mathrm{eV}, \mathrm{rf}-17-\mathrm{dEpg}_{\sim 300 \mathrm{~K}}(300$ th)- - $0.058(1)$. This redshift is much higher than the initial Urbach energy in the pristine layer, $\sim 0.018 \mathrm{eV}$. Its value is about those of the Urbach energy induced by aging in Ajd-rf-f0, 52-59(2) eV. This suggests that illumination under air generates defects in rf-17 that give rise to a distribution of shallow electronic levels.

In addition, illumination is associated in $\mathrm{rf}-17$ to a strong photo-quenching of the PL intensity. One question is whether light produces two types of defects in rf-17, those at the origin of the broadening of the band tails and those responsible for the intensity photo-quenching. The small decrease of the energy and intensity between the 30th and 300th, $-20 \%$ and $10 \%$, respectively, shows that both distributions are mainly produced during the first 30 min of illumination.

Light illumination effects have been extensively investigated in HOIPs in the recent years. Results give evidence that different behaviors are reported depending on the illumination conditions (atmosphere, temperature) and on HOIPs composition and types (crystals, layer/substrates, encapsulated layer/ substrates, active layers in solar cells, etc.) [34].

In $\mathrm{MAPbI}_{3}$, for example, illumination at room temperature is reported to induce an increase of the PL peak intensity in layer/glass under $\mathrm{N}_{2}$ and air atmosphere $[10,35]$ or in single crystals under air or argon atmosphere (Tian2015). The origin of this so-called light soaking effect is attributed to lightinduced iodide migration [35] or to oxygen induced-curing of defects [11]. In the first case [35], using Time-of-flight secondary ion-mass spectroscopy (ToF-SIMS), the authors demonstrate that iodide ions migrate away from the laser spot during illumination. The PL peak energy remains constant under illumination. The PL intensity at the laser spot can be reversibly restored in dark. The authors propose that a charge space induced by the laser illumination creates a local electrical field that drifts iodide ions away from the illuminated region. In the second case [11], the PL intensity increase is reported for illumination of crystals in air. The authors attribute the PL intensity enhancement with illumination-time to a photochemical reaction involving oxygen which passivates deep-lying trap defects.

In mixed halide HOIPs where the $\mathrm{X}$ site is occupied by a mixing of I- and Br- anions, illumination is reported to shift the PL peak energy to lower energies [12, 36-38]. This effect referred as the "Hoke effect" is observable for light intensities higher than 1 sun [34] and is reversible in dark when the light is switched off. The physical origin of this effect is under debate. Local heating induced by laser illumination is excluded $[34,38]$. For some authors, light induces a halide segregation resulting in the formation of iodide-rich and bromide-rich domains which have different bandgaps $[36,37]$. The lower ones are associated to Iodide rich domains that trap carriers. For others, it is a defect-assisted mechanism where halide ions migrate away from the light-excited HOPIs surface via halide defects [12, 38]. An investigation of the dependence of the "Hoke effect" with the Br/I stoichiometry of the solution used for the solution-process growth indicates that it is present for solution that have a $\mathrm{Br}$ content greater than $20 \%[3,34]$. The role of excess halide precursor during preparation has also been considered as playing a role [38]. The role of atmosphere has also been investigated in $\operatorname{MAPb}\left(\mathrm{Br}_{0.5} \mathrm{I}_{0.5}\right)_{3}$ /quartz and PMMA/MAPb $\left(\mathrm{Br}_{0.5} \mathrm{I}_{0.5}\right)_{3} /$ quartz layers [12]. The repetition of illumination(15 s)/dark(30 min) cycles in vacuum, air and nitrogen atmosphere shows that the PL spectra are composed of different PL peaks with energy and intensity evolving with the number of cycles. The peaks in the range, 1.610/(770)$1.722 /(720)(\mathrm{eV} /(\mathrm{nm}))$, are attributed to iodide-rich phase. Those in the range, $1.878 /(660)-1.937 /(640)(\mathrm{eV} /(\mathrm{nm}))$ are attributed to bromide-rich phase. For the lower energy peaks, there is an increase of the PL intensity with the number of illumination cycles independently on the atmosphere. The illumination-induced ( $15 \mathrm{~s}$ ) variations reversibly evolves during the dark period (30 min). The reversible effect is stronger for illumination under inert nitrogen and PMMA covered layers.

In the present work, the PL peak energy decrease with increasing illumination time is observed in the $\mathrm{GA}_{0.015} \mathrm{Cs}_{0.046} \mathrm{MA}_{0.152} \mathrm{FA}_{0.787} \mathrm{~Pb}\left(\mathrm{I}_{0.815} \mathrm{Br}_{0.185}\right)_{3}$ HOIP rf-17 layer obtained via a solution growth process from a solution with a nominal bromide content of $18.5 \%$, and for an illumination intensity higher than 1 sun. The light intensity corresponds to the conditions reported in literature $[3,34]$ for such a process to occur while the bromide content in the solution is slightly lower. It is worth noting that the PL spectra are single peak in the pristine rf-17 quadruple cation and double mixed halide (I,Br) HOIP and double peaks in the $\mathrm{MAPb}\left(\mathrm{Br}_{0.5} \mathrm{I}_{0.5}\right)_{3}$ 
single cation double mixed halide (I,Br) HOIP [12]. The energy with increasing illumination shifts in rf-17 from $1.6426(5)$ to 1.6041 (5) eV during the first 30 min of illumination and, further, to $1.5865(5) \mathrm{eV}$ during the $300 \mathrm{~min}$. This last energy is lower for ex. than any of those reported for the light-induced evolution in $\mathrm{MAPb}\left(\mathrm{Br}_{0.5} \mathrm{I}_{0.5}\right)_{3}$ [12]. One another striking property in $\mathrm{rf}-17$ is the difference in the time scale evolution for the FHWM PL peak and those for the energy and intensity PL peak. The PL peak FHWM in rf-17 goes on evolving on long illumination timescale $(300 \mathrm{~min})$ whereas the PL peak energy and intensity in rf-17 tend to evolve towards quasi-stationary after much shorter time scale, $30 \mathrm{~min}$ instead of $300 \mathrm{~min}$.

I n s um mary, the pristine rf-17 $\mathrm{GA}_{0.015} \mathrm{Cs}_{0.046} \mathrm{MA}_{0.152} \mathrm{FA}_{0.787} \mathrm{~Pb}\left(\mathrm{I}_{0.815} \mathrm{Br}_{0.185}\right)_{3}$ HOIP layer has an illumination time induced redshift of the PL peak spectra consistent with those previously reported for other mixed (I,Br) halide HOIPs with higher nominal Br content, $\geq \sim 20 \%$ $[3,34]$. The long and high power density illumination in $\mathrm{rf}-17$ under air at $300 \mathrm{~K}$ has strong effects - a PL peak redshift ( $58 \mathrm{meV}$ ) associated to a PL FHWM broadening (+ $100 \mathrm{meV})$ and PL intensity photoquenching $(-96 \%)$. Whether under dark after such an illumination, the PL spectra in rf-17 recover their initial state remains an open question.

\section{Continuous illumination in the Ajd_rf-fo layer (454 nm, 1.1 W/} $\mathrm{cm}^{2}$, air, $\left.300 \mathrm{~K}\right)$ The light intensity in the aged reference Ajd_rf-f0 layer $-1.1 \mathrm{~W} / \mathrm{cm}^{2}$ (11 sun)- is about three orders of magnitude less than in the pristine rf-17 layer $-2548 \mathrm{~W} / \mathrm{cm}^{2}$ $(25,480 \mathrm{sun})$. The peak decrease between 3 and $30 \mathrm{~min}$ is however of the same order in both layers -only about twice smaller in A20d_rf-f0 than in rf- $17,0.007 \mathrm{eV}$ instead of $\sim 0.015 \mathrm{eV}$. The photoquenching of the peak intensity also remains of the same order, approx. $-60 \%$ instead of approx. $-90 \%$. The striking difference is that the 30 min illumination induces opposite FWHM variations of an order of magnitude less in A20d_rf$\mathrm{f} 0$ than in rf-17, approx. $-0.001 \mathrm{eV}$ instead of $\sim 0.010 \mathrm{eV}$.

With increasing duration, $20 \mathrm{~d}(\mathrm{~A} 20 \mathrm{~d}-\mathrm{A} 0 \mathrm{~d}), 53 \mathrm{~d}(\mathrm{~A} 53 \mathrm{~d}-$ $A 0 d)$, and $110 d(A 110 d-A 0 d)$, the decrease of the PL( $\left.{ }^{\text {th }}\right)$ peak energy and FWHM in the Ajd_rf-f0 layer remains monotonous with increasing illumination time. The photoquenching of the peak intensity is however strongly reduced with the peak intensity between 3 and 30 min illumination decreasing by approx. $-60 \%$ for A20d to, only, approx. $-10 \%$ for A53d and approx. $-30 \%$ for A110d.

When illumination starts, the Ajd_rf-f0 aged reference layer differs from the pristine rf-17 one by the width of the aginginduced Ajd band tails. The associated Urbach energies are 53(2) $\mathrm{meV}$ (Table 3) in A20d rf-f0 and 18(2) $\mathrm{meV}$ in rf-17. In addition, there is a sub-bandgap distribution of deep levels present in the Ajd-rf-f0 layer that is absent in rf-17. The 30 min illumination generates similar effects in the Ajd-rf-f0 and rf-17 as concern the evolution of the PL peak energy and intensity. A red-shift of the emission transition is associated to its photoquenching under illumination. Furthermore, the PL peaks are composed of overlapping emission transitions that evolves in both cases under illumination. The photoquenching damping under illumination is independent on the initial Ajd_rf-f0/A20d_rf-f0 peak intensity ratio as aging increases. It is however minimum for the A53d aging where this ratio goes through a minimum. Plausible reason to these evolutions is that illumination generates defects. These illuminationinduced defects give rise to near band edge distribution of shallow electronic states that act as radiative recombination centers with decreasing emission energy.

Continuous illumination in the Ajd_fn layers The A0d fn- $c_{i r}$ concentrations of irradiation defects have long term effects on the Ajd_fn $(j(d)=20,53,110)$ illumination-time dependence of the PL spectra that last over at least $110 \mathrm{~d}$.

(i) The PL spectra in the Ajd_fn aged irradiated layers have Ajd_fn illumination-time dependence that, after each Ajd aging, depends on the A0d_fn- $\mathrm{c}_{\text {ir }}$ initial concentrations.

(ii) The concentrations affect also the correlations between the illumination-induced variations of the Ajd_fn peak energy, FWHM and intensity.

(iii) The evolution of the correlations is reflected in the evolution of the Ajd_fn PL spectra deconvolution into several emission components.

The effects of the A0d_fn irradiation defects on the PL peak deconvolution are illustrated in Fig. 8 for the last Ajd_fl/f4 $\mathrm{PL}(6 \mathrm{th})$ spectra recorded respectively for the $\mathrm{fl}$ and $\mathrm{f} 4$ fluences at the end of the 30-min illumination after each Ajd aging. The deconvoluted Gaussian components - number, emission energy, FHWM, height — evolve as the A0d_fn-c $\mathrm{c}_{\text {irr }}$ concentration of irradiation defects increases from the lower concentration, A0d_f1- $c_{\text {irr }}$ to the higher one, A0d_f4- $c_{\text {irr }}$

With increasing duration, $20 \overline{\mathrm{d}}(\mathrm{A} 20 \mathrm{~d}-\mathrm{A} 0 \mathrm{~d}), 53 \mathrm{~d}(\mathrm{~A} 53 \mathrm{~d}-$ A0d), and 110d(A110d-A0d), the Ajd_f1-PL(6th) spectrum is deconvoluted into three emission components for the A20d_f1 PL(6th) peak (Fig. 8a) and two for the A53d/110d_fl ones (Fig. 8b, c). For the Ajd_f4-PL(6th) spectrum, the deconvolution yields two components for the A20d_f4-PL(6th) peak (Fig. 8d) and three for the A53d/110d_f4-PL(6th) one (Fig. 8e, f). The main Gaussian components with a peak height, Ajd_fn-Hp $>0.6$, have peak energies that decrease with increasing Ajd aging for $\mathrm{fl}$ and go through a weak maximum for $\mathrm{f} 4$. The values in $\mathrm{eV}$ are respectively (Table 6):

A20d_f1-1.586; A53d_f1-1.572; A110d_f1-1.565. A20d_f4-1.559; A53d_f4-1.574; A110d_f4-1.570. 
Fig. 8 Deconvolution in Gaussian emission components of the 6th PL spectra recorded after aging and 30 min of illumination in (GACsMAFA) $\mathrm{Pb}(\mathrm{IBr})_{3}$ HOIPs for the $1 \mathrm{MeV}$ e-irradiated Ajd_fn layers at the $\mathrm{fl}$ and $\mathrm{f} 4$ fluences.

The conditions for the Ajd aging are $\mathrm{j}(\mathrm{d})=20 / 53 / 110$ under $\mathrm{N}_{2}$ atmosphere in dark at $\sim 300 \mathrm{~K}$. Two cycles of $15 \mathrm{~min}$ of illumination-light (15 min); dark (3 min); light (15 min)-are performed before the 6 th spectra recording

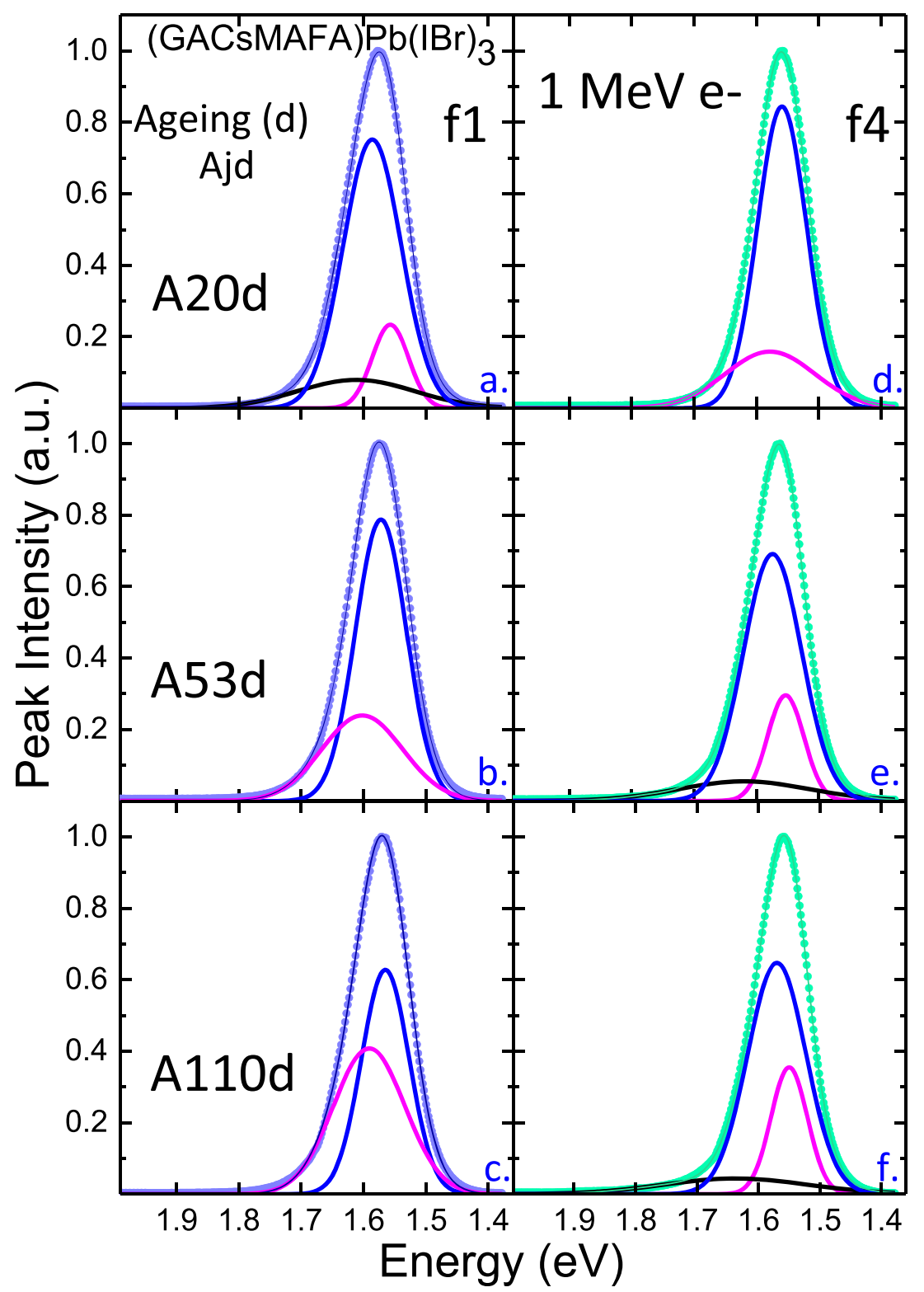

To locate the ionization levels associated to the emission energy, the assumption is that the Ajd_fn bandgaps remain unchanged under $30 \mathrm{~min} 454 \mathrm{~nm}$ air illumination. The A20d_fn ionization levels contributing to the A20d_fn radiative recombinations are located within $44 \mathrm{meV}$ from the conduction/valence band extrema in the $\mathrm{A} 0 \mathrm{~d}$ fl layer and $71 \mathrm{meV}$ in the A0d f4 one. It follows that the higher A0d $\mathrm{f} 4-\mathrm{c}_{\mathrm{ir}}$ irradiation defect concentration favors A20d fn shallow electronic levels that are $(-0.027) \mathrm{eV}$ deeper in the bandgap for the A20 $\mathrm{f} 4$ layer than for the A20d fl one. With further increasing aging duration, the Ajd fn ionization levels contributing to radiative recombinations remain located within $\sim 44(3) \mathrm{meV}$ from the conduction/valence band extrema in the A53d/A110d f1 layer. They become much shallower, within $\sim 26$ and $\sim 10 \mathrm{meV}$ from the conduction/valence band extrema, in the A53d_f4 and A110d_f4 layer, respectively.
Effect of irradiation defects on decay lifetimes and their aging dependence After each Ajd aging $(\mathrm{j}(\mathrm{d})=20,53,110)$, the Ajd_rf-f0/_fn TRPL spectra are recorded in the aged Ajd_rff0 reference and Ajd_fn irradiated layers after the $3 \mathrm{~min}$ in dark at the end of the continuous $30 \mathrm{~min}$ illumination at $454 \mathrm{~nm}$ in air. The TRPL energy windows are chosen with appropriate centers and widths to include the peak energy of the Ajd_rf-f0/_fn-PL( $\left.\mathrm{n}^{\text {th }}\right)$ spectra, $4 \leq n \leq 6$. The Ajd_rf-f0/_fn TRPL spectra are recorded over spots that are within the larger Ajd_rf-f0/_fn PL spots where the Ajd_rf-f0/ fn-PL(1st-6th) spectra are recorded. One considers that the Ajd rf-f0/ fnTRPL spectra are recorded for layer states that are the same as during the recording of the $\mathrm{Ajd} \mathrm{rf}-\mathrm{f0} / \mathrm{fn}-\mathrm{PL}$ (6th) spectra. This is valid as far as the 3 min dark lets quasi-unperturbed the layer state obtained at the end of the recording of the Ajd rf$\mathrm{fO} / \mathrm{fn}-\mathrm{PL}(6 \mathrm{th})$ spectra. Given that this is the case for the first 
Table 6 Deconvolution of the 6th photoluminescence spectra into Gaussian emission components after aging in (GACsMAFA) $\mathrm{Pb}(\mathrm{IBr})_{3}$ HOIPs for the $1 \mathrm{MeV}$ e-irradiated Ajd fn layers at the $\mathrm{fl}$ and $\mathrm{f} 4$ fluence.
The conditions for the Ajd aging are $\mathrm{j}(\mathrm{d})=0 / 53 / 110$ under $\mathrm{N}_{2}$ atmosphere in dark at $\sim 300 \mathrm{~K}$. Epi, FWHM, Hpi@Epi are respectively the peak position, FWHM, and ratio height for the $\mathrm{i}^{\text {th }}$ component

\begin{tabular}{|c|c|c|c|c|c|c|c|c|c|}
\hline $\begin{array}{l}\text { Ajd_fn-Epi } \\
\text { /FWHMi/ } \\
\text { Hpi@Ep }\end{array}$ & Ep1 $(\mathrm{eV})$ & FWHM1 (eV) & Hp@Ep1 & Ep2 $(\mathrm{eV})$ & FWHM2 (eV) & Hp@Ep2 & Ep3 $(\mathrm{eV})$ & FWHM3 $(\mathrm{eV})$ & Hp@Ep3 \\
\hline A20d-f1 & 1.557 & 0.097 & 0.182 & 1.586 & 0.155 & 0.740 & 1.612 & 0.309 & 0.074 \\
\hline A53d-f1 & 1.572 & 0.136 & 0.784 & 1.602 & 0.216 & 0.220 & - & - & - \\
\hline A110d-f1 & 1.565 & 0.127 & 0.618 & 1.591 & 0.191 & 0.385 & - & - & - \\
\hline A20d-f4 & 1.559 & 0.131 & 0.844 & 1.578 & 0.240 & 0.152 & - & - & - \\
\hline A53d-f4 & 1.553 & 0.098 & 0.283 & 1.574 & 0.155 & 0.667 & 1.620 & 0.348 & 0.047 \\
\hline A110d-f4 & 1.550 & 0.099 & 0.343 & 1.570 & 0.158 & 0.626 & 1.637 & 0.390 & 0.035 \\
\hline
\end{tabular}

3 min dark period between the Ajd rf-f0/ fn-PL(3rd) and Ajd_rf-f0/_fn-PL(4th) spectra, it is also most likely the case between the recording of the Ajd_rf-f0/_fn-PL(6th) and of that of the Ajd_rf-f0/_fn-TRPL spectra. The applied light power density has an average value much lower for the TRPL pulsed illumination than for the PL continuous illumination: $3 \times$ $10^{-4} \mathrm{~W} / \mathrm{cm}^{2}\left(\sim 3 \times 10^{-3}\right.$ sun $)$ instead of $1.1 \mathrm{~W} / \mathrm{cm}^{2}(\sim 11$ sun). It is valid to correlate the decay lifetime components resolved in the Ajd_rf-f0/_fn-TRPL spectra to the emission transitions deconvoluted in the Ajd_rf-f0/ fn-PL(6th) as far as the dominant populated ionization levels are the same under the TRPL and PL illumination conditions. It is assumed to be so hereafter although there is one order of magnitude difference between the respective light intensities. This is consequently to be considered as a tentative correlation for the determination of which emission transitions are associated to the longer decay lifetimes in the aged Ajd_rf-f0 reference and Ajd_fn irradiated layers.

The average lifetimes associated to the Ajd_rf-f0/_fn-TRPL spectra have values in $\mu \mathrm{s}$, Ajd_rf-f0/_fn- $\left.<\tau_{\mathrm{Pn}}\right\rangle_{\mathrm{Pn}}: 2.0-4.5$. This reflects the exceptionally long lifetimes associated to the longer lifetime decay component resolved in the Ajd_rf-f0/_fn-TRPL spectra. The lifetime and intensity associated to this longer component vary over ranges in $(\mu \mathrm{s} ; \%)$, Ajd_rf-f0/ fn- $\left[<\tau_{2}>_{\text {Pn }} ;<\mathrm{I}_{2}>_{\text {Pn }}\right]-[\sim(2.8-6.9) ;(26-49)]$. The shorter Ajd_rf-f0/_fn- $\left.<\tau_{1}\right\rangle_{\text {Pn }}$ lifetimes vary over a much lower range, $\sim 0 . \overline{7} 4-2.0 \mu \mathrm{s}$, with intensity always higher than $\sim 50 \%$.

It is interesting to notice that, after the 30 min illumination in the Ajd_fn aged irradiated layers irradiation-induced aging defect populations, the average lifetime, the short and long lifetimes vary non-monotonously with the A0d fn- $\mathrm{c}_{\mathrm{ir}}$ irradiation defect concentrations initially introduced in the fn irradiated layers. The most puzzling is that the higher A0d f4- $\mathrm{c}_{\mathrm{ir}}$ irradiation induces a steep increase for the three of them and for the $\mathrm{I}_{2}$ intensity of the longer lifetime component. They all then reach their maximum values, respectively in $\mathrm{ns}$, A20d_f4-[ $\left[\tau_{\mathrm{Pn}}\right\rangle_{\mathrm{Pn}},\left\langle\tau_{1}\right\rangle_{\mathrm{Pn}},<\tau_{2}>_{\mathrm{Pn}^{-}} \sim[4500,2000,6900]$ and in $\%, A 20 \mathrm{~d} f 4-<\mathrm{I}_{2}>_{\mathrm{Pn}}-50$. This is no longer the case for the A53d and A110d aging where the A0d fn- $\mathrm{c}_{\text {ir }}$ dependence of the lifetimes and intensity have different characteristics. The complex aging behavior of the Ajd_fn- $\left\langle\tau_{\mathrm{Pn}}\right\rangle_{\mathrm{Pn}}-$ $\left.<\tau_{1}>_{\text {Pn }}-<\tau_{2}>_{\text {Pn }}-<\mathrm{I}_{1}>_{\text {Pn }}-\left(1-<\mathrm{I}_{1}\right\rangle_{\text {Pn }}\right)$ lifetimes and intensities confirms that illumination-induced processes after each aging are dependent on the $\mathrm{AOd}$ fn- $\mathrm{c}_{\mathrm{ir}}$ concentration of irradiation defects initially introduced in the layers. The nature of the ionization levels involved in the radiative recombination under the $482 \mathrm{~nm}$ pulsed illumination seem to evolve the less for the higher A0d $\mathrm{fn}-\mathrm{c}_{\mathrm{ir}}$ concentration of irradiation defects $\mathrm{f} 2 \leq$ $\mathrm{fn} \leq \mathrm{f4}$ after the A53d and A110d aging.

For the A20d aging, it is possible to examine the correlation as a function of increasing A0d fn-cir irradiation defects between the evolution of (i) the deconvolution of the PL spectra into Gaussian emission transitions and (ii) the resolution of the TRPL into exponential lifetime components. This correlation shows that the energy decrease, $\sim 0.025 \mathrm{eV}$, from A20d_f11.586 to A20d_f4-1.559 eV (Table 5) corresponds to theseen above - strong increase of the lifetimes and intensity of the longer lifetime. It follows that the main transition in the A20d_f4 layer is characterized by the energy and the decay lifetime, A20d_f4-[Ep, $\left.<\tau_{2}>_{\text {Pn }}\right](\mathrm{eV}, \mathrm{ns})-(\sim 1.559 ; \sim 6900)$.

The correlation between (i) and (ii) as a function of increasing aging duration yields to the determination of the characteristics - peak energy and decay lifetime - of the Ajd_fl/f4 emission transitions in the aged Ajd_fl and Ajd$\mathrm{f} 4$ irradiated layers. As aging duration increases by $47 \mathrm{~d}$ (A53dA110d), for both A0d_fl- $\mathrm{c}_{\mathrm{ir}}$ and A0d-f4- $\mathrm{c}_{\mathrm{ir}}$ concentrations, the energy of the main emission remains quasi the same whereas the average decay lifetime $\left\langle\tau_{\mathrm{Pn}}\right\rangle_{\mathrm{Pn}}$ increases by $63 \%$ for A0d_fl- $\mathrm{c}_{\mathrm{ir}}$ and $9 \%$ for A0d f4- $\mathrm{c}_{\mathrm{ir}}$. The respective characteristics of the A53d/A110d_f1 emission transitions associated to the longer lifetimes are A53/A110d_f1-[Ep; $\left.<\tau_{2}>_{\text {Pn }}\right](\mathrm{eV}, \mathrm{ns})-\sim[1.568(1) / " ; \sim 4121(102) / 5279(404)]$. Those for the A53d/A110d_f4 ones are A53/A110d_f4-[Ep; $\left.\left.<\tau_{2}>_{\mathrm{Pn}}\right](\mathrm{eV}, \mathrm{ns})-\sim\left[1.562(1) /{ }^{\prime \prime} ; \sim 4983(496)\right] / 5695(164)\right]$.

The photocarrier recombination dynamics in absence of local electrical fields after a laser pulse depend on the initial 
density of photoionized carriers $\mathrm{n}(0)$ and on the radiative and non-radiative available recombination channels [39, 40]. The charge carrier dynamics is generally considered to be dominated by trapping at non-radiative recombination centers in HOIPs for photoionization regime where $n(0)$ is much smaller than these center concentration, $\mathrm{N}_{\mathrm{nr}}$ [41-43]. According to various reports, the $\mathrm{N}_{\mathrm{nr}}$ density in pristine MAPI thin films is reported to be around $10^{16-17} \mathrm{~cm}^{-3}$ [41] and around $10^{15} \mathrm{~cm}^{-3}$ for other mixed halide perovskites [44]. For the photoexcitation conditions in this work, given the laser energy per pulse applied, spot and wavelength, the density is expected to be $\sim 10^{13} \mathrm{~cm}^{-3}$ for the initial photoionized carrier density. It is consequently lower than the density for the nonradiative centers previously reported in pristine HOIPs and those expected in the aged Ajd_fn irradiated layers.

The predominant decay lifetime in the A20d_rf-f0 aged reference layer, $4.2 \mu \mathrm{s}$, is longer than those previously reported, $\sim 3.1 \mu \mathrm{s}$, in $\mathrm{GA}_{0.25} \mathrm{MA}_{0.75} \mathrm{PbI}_{3}$ pristine layer on glass [45]. This may reflect the difference in the illumination conditions before the measurements of the decay lifetimes. In the present work, there is $30 \mathrm{~min}$ of $454 \mathrm{~nm}$ continuous illumination in air at $\sim 300 \mathrm{~K}$ before the TRPL measurements. It might also reflect the difference in aging after preparation. One notices indeed that there is a systematic increase of the shorter and longer carrier lifetimes in Ajd_rf-f0 as aging increases from $27 \mathrm{~d}(\mathrm{~A} 20 \mathrm{~d})$ to $117 \mathrm{~d}(\mathrm{~A} 110 \mathrm{~d})$ after their preparation: Ajd_[ $\left.\left\langle\tau_{1}\right\rangle_{\text {Pn }},\left\langle\tau_{2}\right\rangle_{\text {Pn }}(\mu \mathrm{s})\right]:$ A20d_ $\sim[1.2$, 4.2]; A110d_ [1.9, 6.8]. It is also associated to an increase of the fraction of ionized carriers that recombine with the longer lifetime.

It has been earlier reported in $\mathrm{MAPbI}_{3}$ layer on glass [42] that the single component decay lifetime recorded at room $\mathrm{T}(\mathrm{K})$ as a function of pulsed excitation intensity is longer for $48 \mathrm{~h} \mathrm{Ar}$ aged states than for fresh ones. Above a threshold of excitation intensity where bimolecular carrier recombination starts to occur for both states, it is no longer the case. Furthermore, for fresh states at a given pulsed excitation intensity corresponding to the monomolecular recombination regime, the radiative decay lifetimes increase as time elapses. This feature is associated to constant PL spectra. It is referred as self-annealing at room temperature, and is attributed by the authors to a decrease of shallow non-radiative defects and unintentional dopants.

In $\mathrm{GA}_{x} \mathrm{MA}_{1-x} \mathrm{PbI}_{3}$, the GA cations appear to play a key role for the existence of long lifetimes $\sim 3.2 \mu$ s [45]. TRPL measurements were performed in air and room temperature for polycrystalline HOIPs on glass substrates including a mix of MA and GA cations, $\mathrm{GA}_{\mathrm{x}} \mathrm{MA}_{1-\mathrm{x}} \mathrm{PbI}_{3}$ with $x$ increasing in the range $[0,0.05,0.1,0.25]$. The long lifetime in the (multicomponent) lifetime spectra associated to the $\mathrm{x}$ values varies in the range $-0.080,0.9,3.1,0.3 \mu \mathrm{s}-$ and goes through a maximum of $3.1 \mu$ s for $x=0.1$. The authors attribute the two orders of magnitude higher lifetime upon GA incorporation at $x=0.1$ to a faster capability of reorientation for GA than MA in $\mathrm{GAx}_{x} \mathrm{MA}_{1-x} \mathrm{PbI}_{3}$. From DFT calculations, it was reported that quicker rotations of the organic cations result in longer lifetimes of the carriers ionized in the conduction/valence band [46]. The cation rotations enable the formation of shallow traps with the ionized carriers, which extends the carrier lifetime.

In summary, the coupling of electron irradiation with subsequent aging exhibits a striking effect on the carrier radiative lifetime at the end of illumination $(30 \mathrm{~min}, 454 \mathrm{~nm}$, $1.1 \mathrm{Wcm}^{-2}$, air, $300 \mathrm{~K}$ ) after 20 days of aging. The higher A0d_f4-cir irradiation defect concentration promotes a carrier lifetime about 2.46 longer than in $\mathrm{rf}-\mathrm{f0}$ with a value reaching $\sim 6.9 \mu \mathrm{s}$. This lifetime increase is associated to a low emission transition, $1.559 \mathrm{eV}$, and a photoquenching of $\sim 20 \%$ with respect to the PL intensity in A0d_rf-fo.

Aging and electron irradiation defects: nature This paragraph discusses the possible nature of the defects that can be generated during the various treatments investigated here $\left(\mathrm{N}_{2}\right.$ atmosphere, dark, $300 \mathrm{~K}$ ) aging, $1 \mathrm{MeV}$ electron irradiation, continuous illumination. It also discusses some properties of their interaction that can be derived from their effects on optical properties.

Clearly, the $\left.\mathrm{GA}_{0.015} \mathrm{Cs}_{0.046} \mathrm{MA}_{0.152} \mathrm{FA}_{0.787} \mathrm{~Pb}_{\left(\mathrm{I}_{0.815}\right.} \mathrm{Br}_{0.185}\right)_{3}$ layers have the usual properties reported in $\mathrm{ABX}_{3}$ compounds where only one type of cations or halide occupies the $\mathrm{A}$ and $\mathrm{X}$ sites [2]. They are prepared in thermodynamically metastable states that slowly evolve towards quasi-equilibrium states that are dependent on the environmental conditions. One-megaelectronvolt electron is used in this work to control intrinsic defects in HOIPs. Little is known in literature about the damage that $1-\mathrm{MeV}$ electron beam produced in HOIPs. There are more reports on the damage observed in vacuum under the beams used for scanning electron microscopy (SEM) at much (5-10 keV) lower energy and higher flux [47] and for transmission electron microscopy (TEM) [48, 49]. On the contrary, the conditions for the 1$\mathrm{MeV}$ irradiation are chosen in this work to preserve the integrity of the layers and prevent their decomposition under irradiation.

The $\mathrm{APbX}_{3}$ crystalline and chemical structure of the set-18 layers has interesting properties as concern the slowing-down of the $1-\mathrm{MeV}$ electron beam. As well documented since decades (for example, see references in [50-52]), the energy transfer from the beam electrons to the target occurs via elastic collisions with target nuclei, and, via inelastic collisions with target electrons. The elastic collisions generate vacancyinterstitials pairs via replacement collision sequences along the crystalline directions with lower threshold energy displacements [53]. The inelastic ones generate electronic excitation or secondary electrons via ionization. In the $\mathrm{GA}_{0.015} \mathrm{Cs}_{0.046} \mathrm{MA}_{0.152} \mathrm{FA}_{0.787} \mathrm{~Pb}\left(\mathrm{I}_{0.815} \mathrm{Br}_{0.185}\right)_{3}$ set-18 layers, the $\mathrm{Pb}$ and $\mathrm{X}(\mathrm{I}, \mathrm{Br})$ sublattices occupied by inorganic elements are sensitive to the nuclear stopping power. The damage on the respective sublattices is the production of $\mathrm{Pb}$ or $\mathrm{X}$ type 
vacancy - interstitial Frenkel pairs with distributed separation distances. The halide sublattice is occupied by a disordered (I, $\mathrm{Br}$ ) halide solid solution with a nominal content of $18.5 \%$ for the halide sites occupied with $\mathrm{Br}_{\mathrm{X}}$. The probability that a replacement collision sequence ends up in a Frenkel pair production decreases with increasing nuclei mass. Consequently, $1 \mathrm{MeV}$ e-irradiation likely produces higher concentrations of halide Frenkel pairs than lead ones in the set-18 layers. $\mathrm{Br}$ being lighter than iodine, the probability of the formation of $\mathrm{Br}$ interstitial-halide vacancy is high. The halide interstitial distribution is expected to be richer in interstitial of the $\mathrm{Br}_{\mathrm{i}}$ type than the nominal percentage of $\mathrm{Br}_{\mathrm{x}}$ located on the halide sites. The A sublattice, mostly occupied by organic molecular cations (MA, FA, GA), is mainly sensitive to the electron stopping power that excites or ionizes them [51]. Both their excitation and ionization are expected to be fast dissipated by interaction with the ionized carriers in the $\mathrm{APbX}_{3}$ valence and conduction band. Calculations are beyond the scope of this work to check that indeed the number of displaced A cations or of their elements (for example, $\mathrm{H}$ ) is rather low.

At $\mathrm{A} 0 \mathrm{~d}$, in the set- 18 layers $7 \mathrm{~d}$ aged after preparation, $1 \mathrm{MeV}$ e-irradiation progressively removes the initial subbandgap distribution of deep electronic levels and replaces them with irradiation-induced ones. Depending on the Fermi level position and cation/halide stoichiometry, the defects associated to deep levels in $\mathrm{MAPbI}_{3}, \mathrm{MAPbBr}_{3}$ and $\mathrm{FAPbI}_{3}$ [54-56] are the deep donor $\mathrm{I}_{\mathrm{Pb}}, \mathrm{I}_{\mathrm{MA}}, \mathrm{Pb}_{\mathrm{Br}}$, antisites, the deep acceptor $\mathrm{I}_{\mathrm{FA}}, \mathrm{Pb}_{\mathrm{I}}$ antisites and the deep acceptor $\mathrm{Pb}_{\mathrm{i}}$ interstitials. The ionization levels are located between $0.2-0.5 \mathrm{eV}$ from the valence band maximum or conduction band minimum. The replacement collision sequences along the halide sublattice direction of minimal threshold displacement have the cleaning effect of removing the deep levels associated to the A cation (MA, FA, GA) occupying antisite location in the halide sublattice, $\mathrm{I}_{\mathrm{A}}$. The replacement collision sequences along the $\mathrm{Pb}$ sublattice directions of minimal threshold displacement can remove the deep levels associated to the halide (I,Br) elements, $\mathrm{X}_{\mathrm{Pb}}$, occupying an antisite location in the $\mathrm{Pb}$ sublattice. They can also introduce deep levels associated to $\mathrm{Pb}_{\mathrm{i}}$ interstitials when succeeding in producing $\mathrm{Pb}$ vacancyinterstitial Frenkel pairs.

The width of the band tails with increasing A0d fn- $\mathrm{c}_{\text {ir }}$ irradiation concentration evolves with a non-monotonous concentration dependence. On the basis of the shallow levels expected in $\mathrm{MAPbI}_{3}, \mathrm{MAPbBr}_{3}$ and $\mathrm{FAPbI}_{3}$ [54-56], this may reflect the introduction of shallow donor $A_{i}(M A, F A, G A)$ interstitials by removal of $A_{x}$ antisites, of shallow donor $V_{X}(X$ : $\mathrm{I}, \mathrm{Br}$ ) vacancies and shallow acceptor $\mathrm{X}_{\mathrm{i}}(\mathrm{X}: \mathrm{I}, \mathrm{Br})$ interstitial by the production of halide vacancy-interstitials pairs as well as the introduction of shallow acceptor $\mathrm{V}_{\mathrm{Pb}}$ vacancies by the production of lead vacancy-interstitials pairs. One question is whether the presence of the guanidinium organic cations, $\left(\mathrm{C}\left(\mathrm{NH}_{2}\right)^{+}\right)_{3}$, can stabilize halide vacancies. Their quicker rotations may enable to locate one hydrogen of the H-N bonding in the vicinity of a vacancy. Furthermore, the resonance of the positive charge in this moiety may enable to locate it away from the halide vacancy when this vacancy is positively charged. Another one is whether the Ajd_fn band tails result in donor or acceptor doping with higher n-type or p-type conduction in the Ajd_fn irradiated layers than in the Ajd_rf-f0 layer or, rather, to donor-acceptor compensation.

The irradiation-induced aging behavior tends to shrink the bandgap with a non-monotonous concentration dependence. The Ajd_fl irradiated layer has bandgap values close to those of the Ajd_rf-f0 reference layer. The bandgaps are lower in the Ajd_fn $(\mathrm{f} 2 \leq \mathrm{fn} \leq \mathrm{f} 4)$ irradiated layers with fluences in the range [f2- f4] than in the aged Ajd_rf-f0 reference one. On the basis that $\mathrm{MAPbI}_{3}$ has a lower bandgap than $\mathrm{MAPbBr}_{3}$ (for ex. [36]), this points out to an irradiation-aging assisted iodine enrichment of the halide distribution on the halide sublattice. One plausible reason is that migrating iodine interstitials, $I_{i}$, preferentially fill the halide vacancies, $V_{X}$, or exchange positions with bromide occupied halide sites, $\mathrm{Br}_{\mathrm{x}}$. This exchange results in iodine relocation on halide sites, $\mathrm{I}_{\mathrm{x}}$ and replaces $\mathrm{I}_{\mathrm{i}}$ interstitials with $\mathrm{Br}_{\mathrm{i}}$ interstitials. It consequently enriches the interstitial populations with $\mathrm{Br}_{\mathrm{i}}$.

Some authors suggest that a unique feature of HOIPs is that any dangling bond-like defect or associated defect complexes will have transition states close to the band edges [57]. If this statement is correct, this suggests that the irradiation-aging assisted broadening of the band tails arise from the formation of dangling bond-like defect or associated defect complexes. This may reflect that the irradiation-aging assisted $\mathrm{Br}$ depletion of the halide distribution on the halide sublattice sites results in halide vacant sites, i.e., Vx.

Under 454/488 nm illumination in air, the set-18 layers evolve towards states that result from the surface reactivity with oxygen, nitrogen, water vapor that are not photolyzed at these wavelengths. The reactions at the surface of the pristine $\mathrm{rf}-17$ and $7 \mathrm{~d}$ aged $\mathrm{rf}-\mathrm{f0}$ references layers are expected to differ given the different wavelength and power density used for the continuous illumination in air. The comparison between the layer evolutions under continuous illumination can be misleading as concern the origin of the differences. This is why it is no further discussed here.

\section{Conclusion}

This work reports the first experimental data on the effects of intrinsic defects on the aging properties of the emergent $\mathrm{GA}_{0.015} \mathrm{Cs}_{0.046} \mathrm{MA}_{0.152} \mathrm{FA}_{0.787} \mathrm{~Pb}\left(\mathrm{I}_{0.815} \mathrm{Br}_{0.185}\right)_{3}$ HOIPs. For this purpose, the optical properties of $\mathrm{GA}_{0.015} \mathrm{Cs}_{0.046} \mathrm{MA}_{0.152} \mathrm{FA}_{0.787} \mathrm{~Pb}\left(\mathrm{I}_{0.815} \mathrm{Br}_{0.185}\right)_{3}$ layers prepared on glass were investigated as a function of aging duration after gradually increasing concentrations of intrinsic 
defects were produced in the HOIP layers through highenergy electron irradiation $(1 \mathrm{MeV})$. Optical absorption, photoluminescence under continuous laser illumination and time resolved photoluminescence performed before and after increasing aging periods $\left(\mathrm{N}_{2}\right.$, dark, $\left.\sim 300 \mathrm{~K}\right)$ up to 110 days confirmed the stabilization effects of intrinsic defects in quadruple HOIPs.

The existence of similar effects remains to be confirmed in $\mathrm{MAPbI}_{3}$ for which solar cells are reported to exhibit radiation resistance to $1 \mathrm{MeV}$ electron irradiation $[6,7]$ or $50 \mathrm{keV}$ or $68 \mathrm{MeV}$ protons [6-8] and in other HOIPs compounds to confirm their ability to sustain space environment as active layers in photovoltaic devices.

The comparison of the absorption properties in aged and pristine $\mathrm{GA}_{0.015} \mathrm{Cs}_{0.046} \mathrm{MA}_{0.152} \mathrm{FA}_{0.787} \mathrm{~Pb}\left(\mathrm{I}_{0.815} \mathrm{Br}_{0.185}\right)_{3}$ layers showed that naturally aging $\left(7 \mathrm{~d}, \mathrm{~N}_{2}\right.$, dark, $\left.300 \mathrm{~K}\right)$ produced two types of aging-induced defect populations affecting the width of the energy distribution of shallow traps that becomes broader than in pristine layers and distribution of deep traps acting as strong absorption centers. The fluences (fin) chosen in this work for the $1 \mathrm{MeV}$ electron irradiation introduce intrinsic defects in $7 \mathrm{~d}$-aged $\mathrm{GA}_{0.015} \mathrm{Cs}_{0.046} \mathrm{MA}_{0.152} \mathrm{FA}_{0.787} \mathrm{~Pb}\left(\mathrm{I}_{0.815} \mathrm{Br}_{0.185}\right)_{3}$ layers in a concentration range that leaves the bandgap in the layers unchanged. With increasing concentration of defects, the absorption and width variations were found to go through maxima indicating that various processes take place during irradiation and subsequent short $\mathrm{A} 0 \mathrm{~d}$ aging $(<1$ day) periods.

In summary, the interplay of high-energy irradiation offers a new platform to engineer intrinsic defects in perovskite materials that in turn impart higher stability and improvement in functional properties. In addition, the change in fundamental properties such as bandgap shrinkage triggered by irradiation and enhanced optical absorption via deep subgap electronic level can provide innovative processing pathways to enhance the photovoltaics characteristics of HOIP devices.

Acknowledgements Authors are grateful to the University of Cologne, Cologne, Germany and Ecole Polytechnique, Palaiseau, France for the financial and infrastructural support. SM and EJ are grateful for the financial support from German Science Foundation (DFG) provided in the framework of the priority program "SPP 2196 - Perovskite semiconductors: From fundamental properties to devices". PA is grateful to the Doctoral School of the Institut Polytechnique de Paris (ED IP Paris) for the financial support.

Funding Information Open Access funding provided by Projekt DEAL.

\section{Compliance with ethical standards}

Conflict of interest The authors declare that they have no conflict of interest.

Open Access This article is licensed under a Creative Commons Attribution 4.0 International License, which permits use, sharing, adaptation, distribution and reproduction in any medium or format, as long as you give appropriate credit to the original author(s) and the source, provide a link to the Creative Commons licence, and indicate if changes were made. The images or other third party material in this article are included in the article's Creative Commons licence, unless indicated otherwise in a credit line to the material. If material is not included in the article's Creative Commons licence and your intended use is not permitted by statutory regulation or exceeds the permitted use, you will need to obtain permission directly from the copyright holder. To view a copy of this licence, visit http://creativecommons.org/licenses/by/4.0/.

\section{References}

1. NREL Best Research-Cell Efficiencies, https://www.nrel.gov/pv/ cellefficiency.html

2. D.A. Egger, A. Bera, D. Cahen, G. Hodes, T. Kirchartz, L. Kronik, R. Lovrincic, A.M. Rappe, D.R. Reichman, O. Yaffe, What remains unexplained about the properties of halide perovskites? Adv. Mater. 30, 1800691 (2018)

3. C.C. Boyd, R. Cheacharoen, T. Leijtens, M.D. McGehee, Chem. Rev. 119(5), 3418-3451 (2019)

4. S. Wu, Z. Li, J. Zhang, T. Liu, Z. Zhu, A. Jen, Efficient large guanidinium mixed perovskite solar cells with enhanced photovoltage and low energy losses. Chem. Commun. 55, 43154318 (2019). https://doi.org/10.1039/C9CC00016J

5. W. Zhang, J. Xiong, J. Li, W.A. Daoud, J. Mater. Chem. A (2019). https://doi.org/10.1039/C9TA01893J

6. Y. Miyazawa, M. Ikegami, H.-W. Chen, T. Ohshima, M. Imaizumi, K. Hirose, T. Miyasaka, iScience 2, 148-155 (2018)

7. Y. Miyazawa, M. Ikegami, T. Miyasaka, T. Ohshima, M. Imaizumi, K. Hirose, IEEE (2015), https://doi.org/10.1109/PVSC.2015. 7355859

8. F. Lang, N.H. Nickel, J. Bundesmann, S. Seidel, A. Denker, S. Albrecht, V.V. Brus, J. Rappich, B. Rech, G. Landi, H.C. Neitzert, Radiation hardness and self-healing of perovskite solar cells. Adv. Mater. 28, 8726-8731 (2016)

9. O. Plantevin, S. Valère, D. Guerfa, F. Lédée, G. Trippé-Allard, D. Garrot, E. Deleporte, Phys. Status Solidi B, 1900199 (2019)

10. D.W. de Quilettes, S.M. Vorpahl, S.D. Stranks, H. Nagaoka, G.E. Eperon, M.E. Ziffer, H.J. Snaith, D.S. Ginger, Science 348(6235), 683-686 (2015)

11. Y. Tian, A. Merdasa, E. Unger, M. Abdellah, K. Zheng, S. McKibbin, A. Mikkelsen, T. Pullerits, A. Yartsev, V. Sundström, I.G. Scheblykin, J. Phys. Chem. Lett. 6(20), 4171-4177 (2015)

12. A.J. Knight, A.D. Wright, J.B. Patel, D.P. McMeekin, H.J. Snaith, M.B. Johnston, L.M. Herz, ACS Energy Lett., 4, 75-84 (2019, 2018)

13. J.A. Guerra, A. Tejada, L. Korte, L. Kegelmann, J.A. Töfflinger, S. Albrecht, B. Rech, R. Weingärtner, Determination of the complex refractive index and optical bandgap of $\mathrm{CH} 3 \mathrm{NH} 3 \mathrm{PbI} 3$ thin films. J. Appl. Phys. 121, 173104 (2017)

14. P. Nandakumar, C. Vijayan, Y.V.G.S. Murti, Optical absorption and photoluminescence studies on CdS quantum dots in Nafion. J. Appl. Phys. 91, 1509-1514 (2002)

15. Ikhmayies2013, S.J. Ikhmayies, R.N. Ahmad-Bitar, J. Mater. Res. Technol. 2(3), 221-227 (2013)

16. B.U. Haq, S. AlFaify, A. Laref, R. Ahmed, F.K. Buttd, A.R. Chaudhrye, S.U. Rehmanf, Q. Mahmood, Ceram. Int. 45, 18073 18078 (2019)

17. S. De Wolf, J. Holovsky, S.-J. Moon, P. Löper, B. Niesen, M. Ledinsky, F.-J. Haug, J.-H. Yum, C. Ballif, J. Phys. Chem. Lett. 5, 1035-1039 (2014)

18. W. Rehman, D.P. McMeekin, J.B. Patel, R.L. Milot, M.B. Johnston, H.J. Snaith, L.M. Herz, Photovoltaic mixed-cation lead mixed- 
halide perovskites: links between crystallinity, photo-stability and electronic properties. Energy Environ. Sci. 10, 361-369 (2017)

19. W. Li, K. Zhao, H. Zhou, W. Yu, J. Zhu, Z. Hu, J. Chu, Precursor solution temperature dependence of the optical constants, band gap and Urbach tail in organic-inorganic hybrid halide perovskite films. J. Phys. D. Appl. Phys. 52, 045103 (2019)

20. I. Studenyak, M. Kranjec, M. Kurik, Int. J. Optics Appl. 4(3), 76-83 (2014)

21. C. Kim, T.D. Huan, S. Krishnan, R. Ramprasad, A hybrid organicinorganic perovskite dataset. Scientific Data 4, 170057 (2017)

22. A.K. Jena, A. Kulkarni, T. Miyasaka, Chem. Rev. 119(5), 3036$3103(2019)$

23. M. Samiee, S. Konduri, B. Ganapathy, R. Kottokkaran, H.A. Abbas, A. Kitahara, P. Joshi, L. Zhang, M. Noack, V. Dalal, Defect density and dielectric constant in perovskite solar cells. Appl. Phys. Lett. 105, 153502 (2014)

24. A.D. Wright, R.L. Milot, G.E. Eperon, H.J. Snaith, M.B. Johnston, L.M. Herz, Band-Tail Recombination in Hybrid Lead Iodide Perovskite. Adv. Funct. Mater. 27, 1700860 (2017)

25. F. Staub, H. Hempel, J.-C. Hebig, J. Mock, U.W. Paetzold, U. Rau, T. Unold, T. Kirchartz, Beyond Bulk Lifetimes: Insights into Lead Halide Perovskite Films from Time-Resolved Photoluminescence. Phys. Rev. Appl. 6, 044017 (2016)

26. P.M. Amirtharaj, D. G. Seiler in Handbook of Optics, $2^{\text {nd }}$ edn. Vol II, ed. By M. Bass (McGraw-Hill, Inc., New York, 1995), p. 36.8

27. G.M. Martin, Optical assessment of the main electron trap in bulk semi-insulating GaAs. Appl. Phys. Lett. 39, 747-748 (1981)

28. U. Zammit, K.N. Madhusoodanan, M. Marinelli, F. Scudieri, R. Pizzoferrato, F. Mercuri, E. Wendler, W. Wesch, Opticalabsorption studies of ion-implantation damage in $\mathrm{Si}$ on sapphire. Phys. Rev. B 49, 14322-14330 (1994)

29. D. Ritter, K. Weiser, Suppression of interference fringes in absorption measurements on thin films. Opt. Commun. 57, 336-338 (1986)

30. A. Larena, F. Millan, G. Pérez, G. Pinto, Effect of surface roughness on the optical properties of multilayer polymer films. Appl. Surf. Sci. 187, 339-346 (2002)

31. J.K. Katahara, H.W. Hillhouse, Quasi-Fermi level splitting and subbandgap absorptivity from semiconductor photoluminescence. J. Appl. Phys. 116, 173504 (2014)

32. A.D. Wright, C. Verdi, R.L. Milot, G.E. Eperon, M.A. PérezOsorio, H.J. Snaith, F. Giustino, M.B. Johnston, L.M. Herz, Electron-phonon coupling in hybrid lead halide perovskites. Nat. Commun. 7, 11755 (2016)

33. L. Malikova, W. Krystek, F.H. Pollak, N. Dai, A. Cavus, M.C. Tamargo, Temperature dependence of the direct gaps of $\mathrm{ZnSe}$ andZn0.56Cd0.44Se. Phys. Rev. B 54, 1819-1824 (1996)

34. D.J. Slotcavage, H.I. Karunadasa, M.D. McGehee, Light-Induced Phase Segregation in Halide-Perovskite Absorbers. ACS Energy Lett. 1, 1199-1205 (2016)

35. D.W. de Quilettes, W. Zhang, V.M. Burlakov, D.J. Graham, T. Leijtens, A. Osherov, V. Bulovic, H.J. Snaith, D.S. Ginger, S.D. Stranks, Nat. Commun. 7, 11683 (2016)

36. E.T. Hoke, D.J. Slotcavage, E.R. Dohner, A.R. Bowring, H.I. Karunadasa, M.D. McGehee, Reversible photo-induced trap formation in mixed-halide hybrid perovskites for photovoltaics. Chem. Sci. 6, 613-617 (2015)

37. S.J. Yoon, M. Kuno, P.V. Kamat, Shift happens. How halide ion defects influence photoinduced segregation in mixed halide perovskites. ACS Energy Lett. 2, 1507-1514 (2017)

38. A.J. Barker, A. Sadhanala, F. Deschler, M. Gandini, S.P. Senanayak, P.M. Pearce, E. Mosconi, A.J. Pearson, Y. Wu,
A.R.S. Kandada, T. Leijtens, F. De Angelis, S.E. Dutton, A. Petrozza, R.H. Friend, Defect-assisted photoinduced halide segregation in mixed-halide perovskite thin films. ACS Energy Lett. 2, 1416-1424 (2017)

39. J. I. Pankove, Optical processes in Semiconductors, $1^{\text {st }}$ edn. (Prentice-Hall, Inc., Englewood Cliffs, N-J, 1971), pp. 107-156

40. J.R. Lakowicz, Principles of fluorescence spectroscopy, 3rd edn. (Springer, New York, 2006), p. 99

41. L.M. Herz, Charge-carrier dynamics in organic-inorganic metal halide perovskites. Annu. Rev. Phys. Chem. 67, 65-89 (2016)

42. Y. Yamada, M. Endo, A. Wakamiya, Y. Kanemitsu, J. Phys. Chem. Lett. 6, 482-486 (2015)

43. S.D. Stranks, V.M. Burlakov, T. Leijtens, J.M. Ball, A. Goriely, H.J. Snaith, Recombination Kinetics in Organic-Inorganic Perovskites: Excitons, Free Charge, and Subgap States. Phys. Rev. Applied 2, 034007 (2014)

44. A. Bercegol, F.J. Ramos, A. Rebai, T. Guillemot, D. Ory, J. Rousset, L. Lombez, J. Phys. Chem. C 122(43), 24570-24577 (2018)

45. D.J. Kubicki, D. Prochowicz, A. Hofstetter, M. Saski, P. Yadav, D. Bi, N. Pellet, J. Lewiński, S.M. Zakeeruddin, M. Grätzel, L. Emsley, J. Am, Chem. Soc. 140(9), 3345-3351 (2018)

46. J. Gong, M. Yang, X. Ma, R.D. Schaller, G. Liu, L. Kong, Y. Yang, M.C. Beard, M. Lesslie, Y. Dai, B. Huang, K. Zhu, T. Xu, J. Phys. Chem. Lett. 7(15), 2879-2887 (2016)

47. C. Xiao, Z. Li, H. Guthrey, J. Moseley, Y. Yang, S. Wozny, H. Moutinho, B. To, J.J. Berry, B. Gorman, Y. Yan, K. Zhu, M. AlJassim, J. Phys. Chem. C 119(48), 26904-26911 (2015)

48. B. Yang, O. Dyck, W. Ming, M.-H. Du, S. Das, C.M. Rouleau, G. Duscher, D.B. Geohegan, K. Xiao, ACS Appl. Mater. Interfaces 8(47), 32333-32340 (2016)

49. S. Chen, X. Zhang, J. Zhao, Y. Zhang, G. Kong, Q. Li, N. Li, Y. Yu, N. Xu, J. Zhang, K. Liu, Q. Zhao, J. Cao, J. Feng, X. Li, J. Qi, D. Yu, J. Li, P. Gao, Atomic scale insights into structure instability and decomposition pathway of methylammonium lead iodide perovskite. Nat. Commun. 9, 4807 (2018)

50. K. Nordlund, S.J. Zinkle, A.E. Sand, F. Granberg, R.S. Averback, R. Stoller, T. Suzudo, L. Malerba, F. Banhart, W.J. Weber, F. Willaime, S.L. Dudarev, D. Simeone, Improving atomic displacement and replacement calculations with physically realistic damage models. Nat. Commun. 9, 1084 (2018)

51. C.N. Shingledecker, R. Le Gal, E. Herbsta, A new model of the chemistry of ionizing radiation in solids: CIRIS. Phys. Chem. Chem. Phys. 19, 11043-11056 (2017)

52. N. Bohr, Phil. Mag. S. 6, 26: 151, 1-25 (1913)

53. A.Y. Konobeyev, U. Fischer, Y.A. Korovin, S.P. Simakov, Evaluation of effective threshold displacement energies and other data required for the calculation of advanced atomic displacement cross-sections. Nucl. Energy Technol. 3, 169-175 (2017)

54. W.-J. Yin, T. Shi, Y. Yan, Unusual defect physics in CH3NH3PbI3perovskite solar cell absorber. Appl. Phys. Lett. 104, 063903 (2014)

55. A. Mannodi-Kanakkithodi, J.-S. Park, D.H. Cao, N. Jeon, A.B.F. Martinson, M.K.Y. Chan, IEEE (2015). https://doi.org/10.1109/ PVSC.2018.8547974

56. N. Liu, C.Y. Yam, First-principles study of intrinsic defects in formamidinium lead triiodide perovskite solar cell absorbers. Phys. Chem. Chem. Phys. 20, 6800-6804 (2018)

57. W. Walukiewicz, I. Rey-Stolle, G. Han, M. Jaquez, D. Broberg, W. Xie, M. Sherburne, N. Mathews, M. Asta, J. Phys. Chem. Lett. 9(14), 3878-3885 (2018) 